\title{
Analysis of Some Electrolyte Models Including Their Ability to Predict the Activity Coefficients of Individual lons
}

Sun, Li; Liang, Xiaodong; von Solms, Nicolas; Kontogeorgis, Georgios M.

Published in:

Industrial and Engineering Chemistry Research

Link to article, DOI:

10.1021/acs.iecr.0c00980

Publication date:

2020

Document Version

Peer reviewed version

Link back to DTU Orbit

Citation $(A P A)$ :

Sun, L., Liang, X., von Solms, N., \& Kontogeorgis, G. M. (2020). Analysis of Some Electrolyte Models Including Their Ability to Predict the Activity Coefficients of Individual lons. Industrial and Engineering Chemistry Research, 59(25), 11790-11809. https://doi.org/10.1021/acs.iecr.0c00980

\section{General rights}

Copyright and moral rights for the publications made accessible in the public portal are retained by the authors and/or other copyright owners and it is a condition of accessing publications that users recognise and abide by the legal requirements associated with these rights.

- Users may download and print one copy of any publication from the public portal for the purpose of private study or research.

- You may not further distribute the material or use it for any profit-making activity or commercial gain

- You may freely distribute the URL identifying the publication in the public portal 


\section{Analysis of Some Electrolyte Models including Their Ability to Predict the Activity Coefficients of Individual Ions}

Li Sun, Xiaodong Liang, Nicolas von Solms, Georgios M. Kontogeorgis*

Center for Energy Resources Engineering

Department of Chemical and Biochemical Engineering

Technical University of Denmark

Søltofts Plads, Building 229

2800 Kgs. Lyngby, Denmark

Keywords: e-CPA, Classical Theories, Activity Coefficients of Individual Ions, Aqueous Solutions 


\section{Abstract}

This work presents a modeling study, with selected models, of the mean ionic activity coefficients of electrolytes in aqueous solutions, where predictions of the activity coefficients of individual ions (ACI) are also considered, as well as an analysis of the various contributions in the models. In this work, we consider one electrolyte equation of state (the electrolyte Cubic-Plus-Association Equation of State, e-CPA) and other approaches based on combinations of various versions of the Debye-Hückel (DH) or mean spherical approximation (MSA) and Born terms. The results obtained in this work for ACI and the analysis of terms are discussed in the context of other recent literature studies. The results with the various modeling approaches are not identical but all models show some qualitatively good behavior for the ACI, with the "extended version of DH+Born" model performing overall better. Moreover, this study shows that, while good results are obtained in many cases, several parameters like the ion radius, Born radius and relative static permittivity have a significant effect on the results. Finally, in all approaches it appears that the ion-ion (DH or MSA) and ion-solvent (Born) terms have almost equally large "opposing" contributions to the activity coefficients, indicating that both are equally important. This conclusion is agreement with most literature studies. 


\section{Introduction and Motivation}

Most of electrochemistry and chemical engineering involves salt solutions (often highly concentrated), where understanding of the activity coefficients of individual ions (ACI), which depends on the concentration of each component in the solution facilitates the understanding of complex aqueous electrolyte systems ${ }^{1}$.

Numerous electrolyte Equations of State (e-EoS) and activity coefficient models suitable for electrolyte solutions have been proposed and they are summarized in various reviews and textbooks (Kontogeorgis and Folas, 2010²; Tan et al., 20083; Thomsen, 2006 ${ }^{4}$; Prausnitz et al., 1999 $)$. It is often quite difficult to evaluate the true capabilities and application range of these models beyond the range of data used in the model development and the estimation of the adjustable parameters. One way to validate the models is to apply them to properties and conditions other than those used in their development. This is rarely done in literature ${ }^{2}$. The true predictive ability of the models is not always easy to assess.

In this work, we analyze some models based on the primitive approach, i.e. where the solvent is not considered explicitly but is characterized via its relative static permittivity. The models we consider are a version of the electrolyte Cubic-Plus-Association Equation of State (e-CPA) as well as various combinations of the Debye-Hückel theory (DH) or Mean Spherical Approximation (MSA) with the Born term. While e-CPA is a "complete" model having both ion-ion and solvation effects (via a Born-term) as well as terms which account for physical and hydrogen bonding interactions, the other category of models we analyze here consider only 
electrostatic type interactions (ion-ion and ion-solvent (in this work, solvent is water)). We use existing parameterizations of the models from literature and we do not refit parameters. Instead, we analyze all models in two ways: i) validating their capabilities in predicting activity coefficients of individual ions, data not used in the models' parameter estimation and rarely used in electrolyte studies and ii) analyzing the relative magnitude of all terms (physical and electrostatic; ion-ion and solvation) in the values of activity coefficients of salt and individual ions.

The results of this study are discussed against the few literature studies where similar investigations have been carried out. The results and discussion provide insights on the relative importance of the diverse contributions of models for electrolyte solutions, assess their capabilities in the sensitive case of ACI and thus contributing to future model development for improved theories for electrolyte solutions.

This manuscript is organized as follows: firstly, literature studies on ACI are summarized, followed by a presentation of the models considered. In section 4 we present the database of ACI and the parameters used in the models. Section 5 presents the results (ACI and analysis of terms), first for models with only electrostatic terms and then for e-CPA. An extensive discussion and some conclusions follow, while supplementary information is available in the Supporting Information.

\section{Literature Studies on Activity Coefficients of Individual Ions}

The investigation of ACI has attracted much attention ${ }^{1,6}$. This is because ACI can be useful in the characterization of transport phenomena in electrolyte systems $\mathrm{s}^{7-8}$ and are 
also helpful in our understanding of biological systems (sodium-potassium pump), membrane processes, as well as salting-in and salting-out effects ${ }^{1,9}$. Moreover, recently, in some modelling studies, ACI have been considered, as discussed below $^{9-17}$.

The usual experimental method for obtaining ACI is via electrochemical cell measurements using ion-selective electrodes (ISE) and liquid junction potential ${ }^{9}$, then applying expressions for the junction potential and Electromotive force (EMF) of the ISE related to the ionic activities ${ }^{9-12}$. The experimental ACI data published in literature are presented in Table S1 of Supporting Information for several inorganic salts. Below we discuss some of the modelling studies where such data are used.

Fraenkel $^{18}$ used MC-UPM (Monte Carlo simulations of the unrestricted PM) and an extension of the DH theory for the case of ions of unequal size (DH-SiS). In Fraenkel's modelling work ${ }^{18}$, selected salts ( $\mathrm{LiCl}, \mathrm{NaCl}, \mathrm{NaBr}, \mathrm{MgCl}_{2}, \mathrm{CaCl}_{2}, \mathrm{~K}_{2} \mathrm{SO}_{4}$ ) in aqueous solutions are studied. Besides system variables and universal constants, only the size of the ion and the closest distance of the ion to its counter-ion are necessary individual parameters for estimating the ACI. Fraenkel ${ }^{18}$ concluded that there is no need to consider ion solvation, ion pairing, and core effects in computing single-ion activities, if what is desired is a simplified correlation between theory and experiment.

Liu and Eisenberg ${ }^{13,19}$ proposed the Poisson-Fermi (PF) model. In this model, the steric effect of water molecules and interstitial voids in the first and second hydration shells play an important role, and the screening and polarization effects of water are 
also included in the model. Liu and Eisenberg ${ }^{13,25}$ applied this model to calculate the ACI of electrolyte solutions with any arbitrary number of ionic species $(\mathrm{NaCl}$ and $\mathrm{CaCl}_{2}$ in aqueous solutions) in a large range of salt concentrations and temperatures. The PF model gives good agreement with experimental data. More recently, Liu and $\mathrm{Li}^{20}$ also presented results showing very good agreement with experimental ACI data for both 1:1 and 2:1 electrolytes using their generalized DH theory of electrolyte solutions (which is an extension of the PF theory of Liu and Eisenberg).

After proposing the Poisson-Nernst-Planck model ${ }^{21-22}$ and PF model ${ }^{23}$, Liu and Eisenberg developed a new model, called Poisson-Nernst-Planck-Fermi (PNPF) ${ }^{24-25}$, for studying ionic transport through biological ion channels. PNPF are coupled equations, which include the Poisson equation for static electricity field, the Nernst-Planck equation for ion transportation and a Fermi-like distribution without calculating the forces between the particles. PNPF can describe flow, and output the calculations of dielectric properties. Similar to the PF model, PNPF can predict ACI accurately. The PNPF model includes the steric effect of all particles, the correlation effect of ions and water molecules, the screening effect of water, as well as the charge/space mechanism in the channel pore ${ }^{24-25}$. The PNPF model can be easily extended to non-equilibrium systems ${ }^{24-25}$.

Valiskó et al. ${ }^{14-17}$ proposed the so-called "ion-ion (II)+ion-water (IW)" (II+IW) theory. The II term was computed from grand canonical Monte Carlo (GCMC) simulations ${ }^{26}$, and the IW term was computed from the Born equation ${ }^{27}$. They ${ }^{14-17}$ applied the II+IW theory for the calculation of ACI for several inorganic salts in aqueous solutions. 
Qualitative agreement with experimental data is obtained. In these calculations, an extended version of the original Debye-Hückel (EDH) model ${ }^{28}$ was applied for aqueous solutions of $\mathrm{NaCl}$ and $\mathrm{CaCl}_{2}{ }^{29}$. The authors ${ }^{17}$ mentioned that $\mathrm{MSA}^{30}$ can also be used as an alternative method for calculating the II term. II+IW results for ACI with this theory are presented in two publications ${ }^{17,31}$ and for various $1: 1,2: 1$ and $3: 1$ electrolytes. The agreement is satisfactory, at least from a qualitative point of view.

The original $\mathrm{DH}$ does not take steric and correlation effects of ions and water into account. The II+IW theory uses the Born term to calculate ion-water interactions, and the DH-SiS theory ${ }^{18}$ takes into account the ion-water interactions by adjusting the ion size in the calculations. The PF theory ${ }^{13}$ and the Monte Carlo simulations include ion-water and ion-ion interactions.

Vera and Wilczek-Vera in Chapter 27 of their recent book ${ }^{11}$ also discussed emerging theories of electrolyte solutions which have been tested against ACI. They mention that "using well-defined, physically meaningful parameters recent theoretical developments have closely reproduced measured ACI". They refer especially to the previously mentioned works of Liu and Eisenberg ${ }^{19}$ and their PF model and the Smaller-Ion Shell Theory of Fraenkel ${ }^{18}$. In Fraenkel's model (extended version of DH, also based on linearized Poisson-Boltzmann equation), as Vera and Wilczek-Vera notice, experimental ACI data for 6 salts are tested. Vera and Wilczek-Vera show in their work ${ }^{10}$ comparisons with Fraenkel theory for several systems and the agreement is very good. Comparisons are also shown ${ }^{10}$ for some systems against the PF theory of Liu and Eisenberg which is more complex than Fraenkel's model, but the agreement 
is even better at higher concentrations. Valisko and Boda also mentioned the II+IW theory ${ }^{16}$ but in the latter case they say that the agreement against the experimental ACI is not as good as with the previous two theories (although we believe that their results are still acceptable).

In this work, in addition to some of the aforementioned models, the e-CPA EoS proposed by Maribo-Mogensen et al. ${ }^{32}$ is also used. All models are presented in the next section.

\section{Thermodynamic Models}

\subsection{The Original Debye-Hückel and Born Terms}

In 1923, Debye and Hückel ${ }^{33}$ proposed a theory, which is a very important achievement in the study of electrolyte solutions. The DH theory is a primitive model (treating solvents as a continuous dielectric medium), which is based on electrostatic interaction between ions. This theory regards solvent as a continuous medium, and the free energy of the system is the sum of coulombic attraction between positive and negative solute ions without considering short-range repulsive forces. The influence of solvent on the free energy of the system is corrected by introducing the relative static permittivity. The DH theory uses the Poisson's equation ${ }^{34}$ of electrostatic principle, while the charge distribution obeys the Maxwell-Boltzmann distribution ${ }^{35}$, which is mainly applied to the non-associated electrolyte solution where only positive and negative ions exist in the system.

In the original DH theory and related models, the interactions between ions and water 
are not described, thus the original DH theory cannot stand alone as a general model for electrolyte solutions. In order to address this limitation, Stokes and Robinson put forward the theory of ionic hydration ${ }^{36}$. They stated that when ions are dissolved in solvent, the solvent molecules are polarized by the electrical charges.

The Born equation ${ }^{27}$ can be used for estimating the electrostatic component of Gibbs free energy of solvation of an ion. It is an electrostatic model that treats the solvent as a continuous dielectric medium.

It is important to note that in the Born equation, $r_{B o r n, i}$ (Born radius of ion $i$ ) does not have, in principle, the same value as the ion diameter used in the $\mathrm{DH}$ ion-ion interaction calculation. It can be obtained from the above equation by writing it up for the case of an infinitely dilute electrolyte.

The relative static permittivity depends on the thermodynamic state (the temperature and concentration of the electrolyte). In our work, the relative static permittivity (concentration-dependent) is used in both the ion-ion interaction calculation (DH term) and ion-water interactions calculation (Born term).

\subsection{An Extended Version of the Original Debye-Hückel Theory}

Some extensions and simplifications have been proposed for the original DH theory in literature. Shilov and Lyashchenko ${ }^{28}$ extended the original DH theory to allow for an arbitrary concentration dependence of the electrolyte solution relative static permittivity. In fact, in the original EDH paper, Shilov and Lyashchenko ${ }^{28}$ presented two parts of contributions (ion-ion interactions and ion-water interactions), the 
ion-water interactions are also calculated using Born theory. In this work, we name the first contribution EDH.

The expression for the ion-ion interaction contribution to ACI is obtained as follows:

$$
\ln \gamma_{i}=-\frac{Z_{i}^{2} e^{2} \kappa_{0} \chi_{1}\left(\kappa_{0}, a\right)}{3 k T \varepsilon_{w}}+\frac{\bar{V}_{i} \kappa_{0}{ }^{3} \sigma_{1}\left(\kappa_{0}, a\right)}{24 \pi N_{A}}
$$

In Eq. (1), $T$ is temperature, $e$ is the electronic charge, $\varepsilon_{w}$ is the static permittivity of pure water, $N_{A}$ is the Avogadro constant, $k$ is the Boltzmann constant, $Z_{i}$ and is charge of ion $I, \bar{V}_{i}$ is the partial molar volume of ion $i$, and $a$ is the distance of closest approach which was assumed to be the sum of the radii $\left(a=r_{\text {cation }}+r_{\text {anion }}\right)$. Eq. (1) expresses the ACI calculated through the Debye charging process. The first part is electrostatic interaction term, and the second part is an osmotic term, which is small and can be neglected.

$\kappa_{0}$ is the usual inverse Debye screening length, defined with the use of relative static permittivity of solvent (with no salt). More details (e.g. functions of $\chi_{1}$ and $\sigma_{1}$ ) can be found in the original publication ${ }^{28}$ and also later in the manuscript.

\subsection{Mean Spherical Approximation}

Combining the statistical theory of the Ornstein-Zernike equation with the integral equation theory is the starting point of the MSA derivation. In $\mathrm{MSA}^{30}$, the model treats ions as charged hard spheres, the solvent as a continuum, and the thermodynamic properties expression is obtained by solving the integral equation.

By using an average diameter of the ions in the solution, the MSA expression for the excess Helmholtz energy can be reduced to a simple, explicit form ${ }^{30}$. In the excess 
Helmholtz energy calculations of MSA, the expression of $\kappa$ (inverse Debye screening length) is the same with that used in the DH theory.

\subsection{Electrolyte Cubic-Plus-Association}

Maribo-Mogensen et al. $^{32}$ extended the CPA EoS, which was proposed by Kontogeorgis et al. ${ }^{37}$, to mixtures containing electrolytes. In e-CPA, the residual Helmholtz energy is given by:

$A^{r}=A^{S R K}+A^{A S S O C}+A^{D H}+A^{B o r n}$

In the right-hand side of Eq. (2), the first two terms are the contributions from CPA, the $A^{S R K}$ contribution of ions considers the volume-exclusion from ions, the $A^{A S S O C}$ contribution of ions accounts for the effects from the solvent association. The remaining two contributions are from the original $\mathrm{DH}$ theory ${ }^{33}$ (for the long-range interaction of the ions) and the Born equation ${ }^{27}$ (for ion solvation), respectively. e-CPA is set up for ions, i.e. the model parameters are by nature ion specific. In order to minimize the number of parameters, a series of assumptions have been made $32,38-39$. For more details of e-CPA and parameter estimation, the reader is referred to Maribo-Mogensen et al. ${ }^{32}$ and Sun et al. ${ }^{38-39}$. Maribo-Mogensen et al. ${ }^{32}$ published the adjustable model parameters for several salts which are used in section 5.2.

\subsection{The II+IW Theory}

\subsubsection{The II+IW Theory}

Valiskó et al. ${ }^{14-17,29}$ proposed the II+IW theory in which they split the excess chemical 
potential into two parts:

$$
\mu_{i}^{E X}=\mu_{i}^{E X . I I}+\mu_{i}^{E X . I W}
$$

where the II and IW terms describe the ion-ion and ion-water interactions, respectively (subscript $i$ refers to an ionic species).

The II+IW theory is developed for activity coefficient calculations (it is not an EoS) and it does not contain any "physical forces" terms.

In Valiskó et al.'s work ${ }^{14-17,29}$, the II term was computed from GCMC simulations. Alternatively the extended version of the original $\mathrm{DH}^{28}$ theory and $\mathrm{MSA}^{17}$ can also be used for the II calculation ${ }^{15}$. The IW term for ion solvation is calculated by the Born equation $^{27}$.

It should be noted that, in the II+IW theory, experimental volume and relative static permittivity for aqueous electrolyte solutions (concentration-dependent values) are used $^{17}$. In Valiskó et al.'s work ${ }^{14-17,29}$, the II term is computed using hard sphere ions with Pauling radii. The IW term is computed using Born radius, which they fitted from the hydration free energy.

Both II and IW terms contain a (salt) composition-dependent relative static permittivity (fitted experimental data to some form of polynomial equation). In other words, there is no adjustable parameter in the II +IW theory.

\subsubsection{Discussion of Literature Studies of the II+IW Theory}

Here, we present a discussion of the literature results for the two II+IW theories mentioned above; by Shilov \& Lyashchenko, and by Valiskó \& Boda and co-workers. 
While none of the two approaches are "complete" engineering electrolyte models (they do not consider physical or quasi-chemical/association interactions; they do not aim to describe a wide range of properties of electrolyte solutions), their study is certainly of great interest. No adjustable parameters are fitted and they provide an analysis of the ion-ion and ion-water interactions and the role of relative static permittivity entirely clear if the composition derivatives of the relative static permittivity are accounted for, something that would have been expected as composition-dependent relative static permittivity are used.

\subsubsection{Discussion of Shilov and Lyashchenko Theory}

It is of interest to mention that Hückel had presented an extension of the original DH theory using a linear concentration dependency of relative static permittivity in $1925^{40}$. He did not pursue this further.

In both the 2015 and the subsequent papers ${ }^{41-43}$, Shilov and Lyashchenko showed that their model can accurately predict mean ionic activity coefficients for various water-salt (halide) solutions up to high concentrations (often up to $5 \mathrm{~mol} / \mathrm{kg}$, even up to $10 \mathrm{~mol} / \mathrm{kg}$ for $\mathrm{LiCl}$ ). This success is attributed to the simultaneous use of the original DH and Born terms and the concentration dependency of the relative static permittivity. They claim that the balance of the two terms is very important for reproducing the well-known (non-monotonic) curvature of the mean ionic activity coefficients (first decreasing with concentration, going via a minimum and then increasing again). 
In the various plots shown for the mean ionic activity coefficient, the Born term is positive and increasing with salt concentration, while DH is negative and decreasing (opposite trend for water activity). The sum of the two reproduces at least qualitatively the data including the minimum of mean ionic activity coefficient and it is a delicate balance of two rather large terms. It is the presence of both terms and the concentration dependency of relative static permittivity that extends the applicability of the DH theory, according to the authors.

Shilov and Lyashchenko (SL) cite Debye having said that his theory "applies much better than it should" and comment that their extension of DH belongs to the family of linearized PB models, which were originally thought to be responsible for the failure of the $\mathrm{DH}$ theory. When the $\mathrm{DH}+$ Born results are compared to experimental values, it is not readily clear whether the disagreement between theory and experiment is due to approximations in the model (e.g. implicit treatment of water via the primitive framework), or approximations in the method (e.g. mean-field treatment), or some combination of the above.

They use the full relative static permittivity expression and in their recent manuscript ${ }^{42}$ they comment that the kinetic depolarization effect (discussed by e.g. Kontogeorgis, Maribo-Mogensen and Thomsen ${ }^{44}$ ) is negligible according to some authors they cite, although they admit that Hubbard and Onsager ${ }^{45}$ claimed that it is of significance. They admit that this remains an open question.

Another aspect of the SL equations is that apparently, the derivatives of relative static permittivity with concentration (which should be included in both the DH and Born 
terms) are neglected, as they are considered to be rather small (no calculations verifying this are shown). In their recent manuscript ${ }^{42}$, they emphasize the need to differentiate relative static permittivity when needing to obtain expressions for the chemical potentials.

SL are mostly interested in understanding phenomena and not fitting parameters, thus they are less concerned with quantitative agreement and more interested in checking whether experimental trends are reproduced. The DH distance of closest approach (DCA) $a$ of the DH term is simply set as the sum of cation and ion radii (from Pauling/Marcus) and the average radius in the Born term is set to $a / 2$, thus assuming some type of solvated diameter. Valiskó \& Boda and co-workers (see discussion later) criticized SL for using the same type of radii in the DH and Born terms. SL can obtain even better results for the mean ionic activity coefficients by a slight fit of the Born radius (e.g. $0.7 a$ for $\mathrm{NaCl}$ instead of $0.5 a$ ). SL tried to use Valiskó \& Boda's approach (obtaining the Born radius from hydration Gibbs energies) but the results are not improved compared to their approach.

Finally they extend for first time their approach to ion pairs ${ }^{43}$, but this extension is not fully predictive. They comment that some degree of ion association must be already incorporated in the DH theory.

The SL notion that the activity coefficient trend of aqueous electrolyte solutions depends on a balance between two terms is not new and Valiskó \& Boda et al. see discussion later, claim the same, also that the two balanced terms are due to ion-ion and ion-water (Born) interactions. Other researchers e.g. Tikanen and Fawcett ${ }^{46-47}$ 
used the MSA model also with concentration-dependent relative static permittivity. They do not use a Born term, although the Born term is very important for ion-water interaction calculations. In the Tikanen and Fawcett's calculations, the hydrated ionic diameter is used instead of the crystallographic ion diameter and by using the hydrated ionic diameter, the ion-ion interactions are weakened. They find good results including the trend of activity coefficients with salt concentration and the minimum by fitting the ion diameter. The agreement is better compared to using constant relative static permittivity. There is again a balance between two large terms, which in their model are the hard-sphere and the ion-ion (electrostatic) interactions. They emphasize the role for ion-pairs at high concentrations. Tikanen and Fawcett are "obsessed" that DH fails at high concentrations and only works for very dilute systems, and thus MSA should be preferred, but they stated that some extended versions of the DH is only valid up to $0.1 \mathrm{M}$ even with fitted size parameter. They never explain which version of DH they have in mind. They even say that if we attempt to use the extended version of DH with concentration-dependent relative static permittivity, no improvement is obtained. Once again, they state all these without showing any results and without explaining which DH they mean. Anyhow, they conclude that in addition to a good ion-ion interaction term, the finite size of ions and the concentration dependency of the relative static permittivity are the important features that must be considered. The DH-based approaches are sometimes considered to be easier to employ for engineers and experimentalists because these approaches are somewhat simpler and they often provide physically well-based adjustable 
parameters.

\subsubsection{Discussion of Valiskó and Boda Theory}

In a series of papers (especially) since 2010 14, 16-17,31, Valiskó \& Boda and co-workers have presented an activity coefficient approach which bears a lot of similarities with the SL theory. It is also essentially an activity coefficient model, aiming predictions of salt solutions. No additional terms are used other than an ion-ion term and an ion-water term, the latter essentially from the Born theory. They also use a concentration-dependent relative static permittivity in both terms and they consider this to be the link between the two terms. We believe that the derivatives of relative static permittivity with concentration are ignored. One important difference from the SL theory is that Valiskó \& Boda et al. use a so-called "adaptive canonical Monte-Carlo (A-GCMC) simulation method" for the ion-ion interactions (it looks like a rather complex approach, iterative, still based on the primitive model for electrolytes), this method provides the chemical potential for prescribed (targeted) concentration $^{48}$. The second difference from SL is the use of different radii in the ion-ion term (Pauling parameters) and the Born term (the latter are predicted directly from experimental Gibbs energy of solvation data). Valiskó \& Boda et al. in an emphatic way, insist on the need of using different well-defined radii in the II and IW terms and they oppose the use of the "solvated" radii concept (e.g. obtained by fitting to experimental activity coefficient data). There are no adjustable parameters in their work and all their results can be considered to be fully predictive. 
Before mentioning the results, it is important to emphasize that, as in the SL work, there are no calculations beyond the classical mean ionic activity coefficients i.e. no solubilities, LLE, mixed solvents, mixed salts, etc.

They have obtained qualitatively good results for many aqueous electrolyte solutions up to rather high concentrations and the shape of activity coefficients versus concentration is explained as a balance of two large terms, a positive Born contribution (increasing with salt concentration) and a negative ion-ion contribution (decreasing with salt concentration). The delicate balance between the two explains the activity coefficient trend; this conclusion is identical to that of SL. Moreover, Valiskó \& Boda et al. present rather good agreement against the ACI from Vera's works and other researchers.

Valiskó \& Boda et al. have in several of their manuscripts and letters to editor commented-compared their theory to other approaches including SL. They admitted their similarity to the SL theory (and showed comparisons proving that) but somehow, they do not think very highly of DH for the ion-ion interactions. The reasons may be the differences in the performances of the theories in different models. $\mathrm{DH}$ is a mean-field theory based on electrostatics, MSA is a statistical mechanical theory, while GCMC is a molecular simulation approach. DH and MSA contain approximations, while GCMC can give exact results. DH and MSA do not consider ion association, while ion association is an output of a Monte Carlo simulation ${ }^{17}$. Valiskó \& Boda et al. still agree with SL on both the use of Born term and the necessity of concentration-dependent relative static permittivity in both terms (ion-ion 
and ion-water) and hope that such approaches can be incorporated in more advanced methods, as they call the e-SAFT approaches in one of their papers. They emphasize physics and there is no parameter estimation in their works based on experimental. activity coefficient data, which is very positive, and for more accurate results, they do mention that non-primitive approaches with explicit water treatment ${ }^{49}$ should be considered.

\section{Database and Parameter Estimation}

There is significant debate in literature about the concept of ACI and the obtained experimental data.

In order to evaluate the experimental data, we calculated the mean ionic activity coefficients from ACI, and compared these calculated values with the experimental mean ionic activity coefficients from literature (obtained from direct measurements or via osmotic activity coefficients). Figure S1 of Supporting Information shows a preliminary evaluation for 9 salts. It can be seen from these figures that the deviations between different data sets are small, and the deviations of mean ionic activity coefficients between calculated values (from single ion data) and the actual experimental data are also small. While this is not an ultimate proof of the correctness of the single ion values, it is a good indication and we proceed with the use of the revised values of the ACI.

The experimental results show that the activity coefficients are different for the anion and the cation in an aqueous solution. For the activity coefficients of $\mathrm{Na}^{+}$, the values 
in aqueous solutions of $\mathrm{NaF}, \mathrm{NaCl}$ and $\mathrm{NaBr}$ are different, because the ACI are affected by the interaction of cation and anion. Similarly, for the activity coefficients of $\mathrm{Cl}^{-}$in aqueous solutions of $\mathrm{NaCl}$ and $\mathrm{KCl}$. Figure $\mathrm{S} 2$ of Supporting Information shows the activity coefficients of $\mathrm{Na}^{+}$and $\mathrm{Cl}^{-}$in different aqueous electrolyte solutions.

In this work, we study 9 aqueous electrolyte solutions at $298.15 \mathrm{~K}: 1: 1$ electrolytes ( $\mathrm{NaCl}$ : sodium chloride, $\mathrm{KCl}$ : potassium chloride, $\mathrm{NaBr}$ : sodium bromide, $\mathrm{NaF}$ : sodium fluoride); 2:1 electrolytes $\left(\mathrm{CaCl}_{2}\right.$ : calcium chloride, $\mathrm{MgCl}_{2}$ : magnesium chloride); 1:2 electrolytes ( $\mathrm{Na}_{2} \mathrm{SO}_{4}$ : sodium sulfate, $\mathrm{K}_{2} \mathrm{SO}_{4}$ : potassium sulphate); 3:1 electrolytes ( $\mathrm{LaCl}_{3}$ : lanthanum chloride).

In this work, the II+IW theory is used for calculations of ACI. We employed the DH term (e-CPA), the EDH term and the MSA term for II calculations. In addition to differences in expression, the values of ion radii $\left(r_{c a l, i}\right)$ are the apparent differences between the different II terms. The DH term (e-CPA) uses the ionic radii of all ions for individual ion calculations, while EDH and MSA use average ion radius.

Table 1 summarizes the free energy contributions (II terms and the IW term) from the different models considered here, while a short description follows for the models we have used in this work, explaining how they are employed here. 
Table 1. The various modeling approaches considered in this work.

\begin{tabular}{cccc}
\hline Term & Model & Contribution & $r_{\text {cal }, i}$ \\
\hline II & DH (e-CPA) $)^{32}$ & $A^{D H}=-\frac{k_{B} T V}{4 \pi N_{A} \sum_{i} n_{i} Z_{i}^{2}} \sum_{i} n_{i} Z_{i}^{2} \chi_{i}$ & $r_{i}$ \\
II & $\mathrm{EDH}^{28}$ & $G^{D H}=-\frac{k_{B} T V}{12 \pi} \kappa_{0}{ }^{3} \tau_{1}\left(\kappa_{0}, a\right)$ & $a / 2$ \\
II & $\mathrm{MSA}^{30}$ & $A^{D H}=-\frac{2 \Gamma^{3} R T V}{3 \pi N_{A}}\left(1+\frac{3}{2} \Gamma_{M S A} \sigma\right)$ & $\sigma$ \\
IW & Born $^{27}$ & $G_{i}^{\text {Born }}=\frac{Z_{i}^{2} e^{2}}{8 \pi \varepsilon_{0} r_{\text {Born }, i}}\left[\frac{1}{\varepsilon_{r}}-\frac{1}{\varepsilon_{w}}\right]$ & $r_{\text {Born }, i}$ \\
\hline
\end{tabular}

In Table $1, A^{D H}$ is II contribution of residual Helmholtz energy, $G^{D H}$ is the II contribution of Gibbs free energy, in e-CPA, the hard-sphere diameter of the ion $i\left(d_{i}\right)$ is used. In EDH, the expression for the ion-ion interaction contribution to ACI is shown in Eq. (1), In this calculation, we eliminate the term with the partial molar volume in Eq. (1).

The ion radii, Born radii and ion-water interaction parameters in e-CPA of several inorganic salts, as used in this work, are presented in Tables S2 and S3 of Supporting Information.

In calculations using the e-CPA EoS, the ion size from Marcus ${ }^{50}$ and Born radii from our previous work ${ }^{32}$ are used. In calculations using the II+IW theory (all the three models use the same physical parameters), Pauling radii ${ }^{51}$ and Born radii from Valiskó et al. ${ }^{16-17}$ are used (for $\mathrm{SO}_{4}{ }^{2-}$ and $\mathrm{NO}_{3}{ }^{-}$, the e-CPA parameters are used). 
In the II + IW theory calculations, experimental liquid density (or correlations of experimental data) are required. From literature, the density of aqueous electrolyte solutions as a function of their temperature and concentration can be expressed as ${ }^{52}$ :

$$
\rho=\rho_{w}+A c+B c t+C c t^{2}+D c^{3 / 2}+E c^{3 / 2} t+F c^{3 / 2} t^{2}
$$

where $A$ through $F$ are adjustable constants (listed in Table S4 of Supporting Information), $c$ is the salt concentration molarity in $\mathrm{mol} / \mathrm{L}, t$ is the temperature in ${ }^{\circ} \mathrm{C}$, and $\rho$ is the density of solution in $\mathrm{kg} / \mathrm{m}^{3}$. The density of water, $\rho_{w}$ is calculated according to the empirical equation ${ }^{52}$ :

$$
\rho_{w}=999.65+2.0438 \times 10^{-1} t-6.174 \times 10^{-2} t^{3 / 2}
$$

For the concentration-dependent relative static permittivity of solvent in aqueous electrolyte solutions, the equations of fitted curves are :

$$
\varepsilon(c)=78.45-\delta_{s} c+b_{s} c^{3 / 2}
$$

The expression of concentration-dependent relative static permittivity is given in Eq. (6), for 2:1 electrolytes, the values $\delta_{s}=34$ and $b_{s}=10$ are used ${ }^{16,29,47}$. For aqueous solutions of $\mathrm{Na}_{2} \mathrm{SO}_{4}$ and $\mathrm{K}_{2} \mathrm{SO}_{4}$, the correlations of relative static permittivity have been carried out in this work and the parameters are listed in Table S5 of Supporting Information.

For the thermodynamic calculations, some important equations used are presented in Section C of Supporting Information.

This work presents ACI calculations at $298.15 \mathrm{~K}$, there are no adjustable parameters in the II+IW calculations, and there is one adjustable parameter (the ion-water interaction parameter at) in the e-CPA EoS calculations. 


\section{Results}

\subsection{Results using the II+IW Theory}

At first, we present calculation results using the II+IW theory and various ways to estimate the II and IW contributions. In this section, the II term is calculated from EDH (as shown in Table 1, proposed by Shilov and Lyashchenko ${ }^{28}$ ), MSA (as shown in Table 1, proposed by Lebowitz and Percus ${ }^{30}$ ), and from DH (e-CPA). The IW term is calculated from Born equation ${ }^{27}$ (see Table 1).

Here, we follow Valiskó et al's approach ${ }^{14-17,29}$ using the experimental correlations of density and relative static permittivity for aqueous electrolyte solutions, and using Pauling radii in the II term calculation, and Born radii in the IW term calculation for all the three models (EDH+Bon, MSA+Bon, $\mathrm{DH}+\mathrm{Bon})$.

Table 2 summarizes the results as relative average deviations between the experimental data and predicted values from different models. It can be seen that in some cases deviations seem large for some systems, one reason being that the values of some activity coefficients are small. Overall, the agreement is often satisfactory, which is a rather impressive result considering that the results are predictive, and moreover the model ignores all physical interactions. 
Table 2. Modeling performance of different approaches for ACI and mean ionic activity coefficients. (“+” indicates activity coefficients of cation, “-" indicates activity coefficients of anion and " \pm " indicates the mean ionic activity coefficients).

\begin{tabular}{|c|c|c|c|c|c|c|c|c|c|c|c|c|c|c|c|}
\hline \multirow{3}{*}{ Salt } & \multicolumn{15}{|c|}{ RAD of activity coefficients (molality-based) [\%] } \\
\hline & $\mathrm{EDH}$ & MSA & DH(e-CPA) & Max m & $\mathrm{Np}$ & EDH & MSA & $\mathrm{DH}(\mathrm{e}-\mathrm{CPA})$ & Max m & $\mathrm{Np}$ & $\mathrm{EDH}$ & MSA & DH(e-CPA) & Max m & $\mathrm{Np}$ \\
\hline & + & + & + & + & + & - & - & - & - & - & \pm & \pm & \pm & \pm & \pm \\
\hline $\mathrm{NaCl}$ & 4.4 & 9.8 & 8.6 & 3.0 & $23^{10}$ & 1.7 & 2.1 & 3.5 & 3.0 & $23^{10}$ & 2.8 & 5.5 & 3.7 & 3.0 & $23^{10}$ \\
\hline $\mathrm{KCl}$ & 10.6 & 4.3 & 7.7 & 3.0 & $23^{10}$ & 4.1 & 5.5 & 3.0 & 3.0 & $23^{10}$ & 3.8 & 1.4 & 3.6 & 3.0 & $23^{10}$ \\
\hline $\mathrm{NaBr}$ & 1.2 & 7.5 & 6.2 & 3.0 & $23^{10}$ & 2.8 & 2.0 & 1.8 & 3.0 & $23^{10}$ & 1.4 & 4.8 & 2.8 & 3.0 & $23^{10}$ \\
\hline $\mathrm{NaF}$ & 1.9 & 4.2 & 2.9 & 1.0 & $7^{9}$ & 3.4 & 3.2 & 5.0 & 1.0 & $7^{9}$ & 1.9 & 0.8 & 1.8 & 0.9834 & $11^{53}$ \\
\hline $\mathrm{CaCl}_{2}$ & 10.5 & 14.4 & 18.3 & 0.955 & $20^{54}$ & 11.2 & 4.2 & 5.3 & 3.0 & $15^{9}$ & 24.5 & 23.8 & 26.1 & 3.0 & $30^{55}$ \\
\hline $\mathrm{MgCl}_{2}$ & 48.2 & $56.4^{\mathrm{a}}$ & $69.1^{\mathrm{a}}$ & 3.0 & $15^{9}$ & 19.1 & 11.6 & 2.0 & 3.0 & $15^{9}$ & 37.7 & 27.7 & $53.4^{\mathrm{a}}$ & 3.0 & $20^{56}$ \\
\hline $\mathrm{Na}_{2} \mathrm{SO}_{4}$ & 38.5 & 8.1 & 19.5 & 3.0 & $15^{9}$ & $83.7^{\mathrm{a}}$ & $51.5^{\mathrm{a}}$ & $409.7^{\mathrm{a}}$ & 2.0 & $7^{57}$ & 49.4 & 4.0 & $88.3^{a}$ & 3.0 & $12^{36}$ \\
\hline $\mathrm{K}_{2} \mathrm{SO}_{4}$ & 8.4 & 0.8 & 6.4 & 0.69 & $11^{9}$ & 39.5 & 25.3 & $67.8^{a}$ & 0.69 & $11^{9}$ & 21.8 & 7.1 & 29.5 & 0.7 & $7^{36}$ \\
\hline $\mathrm{LaCl}_{3}$ & $77.2^{\mathrm{a}}$ & $75.6^{a}$ & $85.7^{\mathrm{a}}$ & 1.4 & $7^{58}$ & 18.0 & 18.4 & 22.8 & 1.4 & $7^{58}$ & 43.2 & 24.7 & 33.9 & 1.4 & $7^{58}$ \\
\hline Overall $^{\mathrm{b}}$ & 22.3 & 20.1 & 24.9 & - & - & 20.4 & 13.8 & 57.9 & - & - & 20.7 & 11.1 & 27.0 & - & - \\
\hline Overall $^{c}$ & 15.5 & 7.0 & 9.9 & - & - & 12.5 & 9.0 & 6.2 & - & - & 20.7 & 11.1 & 14.5 & - & - \\
\hline
\end{tabular}

a. outliers ( $\geq 50$ ), EDH: $\mathrm{SO}_{4}{ }^{2-} / \mathrm{Na}_{2} \mathrm{SO}_{4}, \mathrm{La}^{3+} / \mathrm{LaCl}_{3}$; $\mathrm{MSA}: \mathrm{Mg}^{2+} / \mathrm{MgCl}_{2}, \mathrm{SO}_{4}{ }^{2-} / \mathrm{Na}_{2} \mathrm{SO}_{4}, \mathrm{La}^{3+} / \mathrm{LaCl}_{3}$; DH (e-CPA): $\mathrm{Mg}^{2+} / \mathrm{MgCl}_{2}, \mathrm{SO}_{4}{ }^{2-} / \mathrm{Na}_{2} \mathrm{SO}_{4}$,

$\mathrm{SO}_{4}{ }^{2} / \mathrm{K}_{2} \mathrm{SO}_{4}, \mathrm{La}^{3+} / \mathrm{LaCl}_{3}, \mathrm{MgCl}_{2}, \mathrm{Na}_{2} \mathrm{SO}_{4} ;$ b. average deviations with all deviations; c. average deviations without outliers. 
In calculations, the results are typically listed as the relative average deviation $(R A D)$ :

$R A D \%=\frac{1}{N p} \sum_{i}^{N p}\left|\frac{y_{i}^{c a l}-y_{i}^{e x p}}{y_{i}^{e x p}}\right| \times 100 \%$

Here, $N p$ is the number of data points, $y_{i}^{\text {cal }}$ represents the calculated results of any property, e.g. activity coefficient, and $y_{i}^{\text {exp }}$ represents the experimental data of the given property.

Figures 1-4 present results for 1:1 electrolyte solutions. We can see that the II+IW theory (all three models) provides good results for the ACI as well as for the mean ionic activity coefficients. At high salt molality, the deviations between experimental data and calculated values are somewhat larger, but overall, the performance is satisfactory even at rather high concentrations.

For 1:1 electrolyte systems, all three models perform well, especially for activity coefficients of anion and mean ionic activity coefficients. The EDH+Born performance is a bit better than the other two models for activity coefficients of cation. From these results, the reproduction of the mean ionic activity coefficients looks as good as the predictions of ACI for 1:1 electrolytes.

The Born and II terms (either the large as DH or MSA) have large "opposing" values which become even more pronounced at high concentrations. It appears that both play an important role in the representation of the electrostatic effects for electrolyte solutions. Despite the differences mentioned above, all models appear to perform (at least from a qualitative point of view) rather similarly and there are rather small 
differences even between DH and MSA (although these differences are higher for cations compared to anions). Overall, we see a satisfactory performance also for ACI, especially considering that the results are predictive. The above qualitative picture is similar for all 1:1 electrolyte solutions considered.

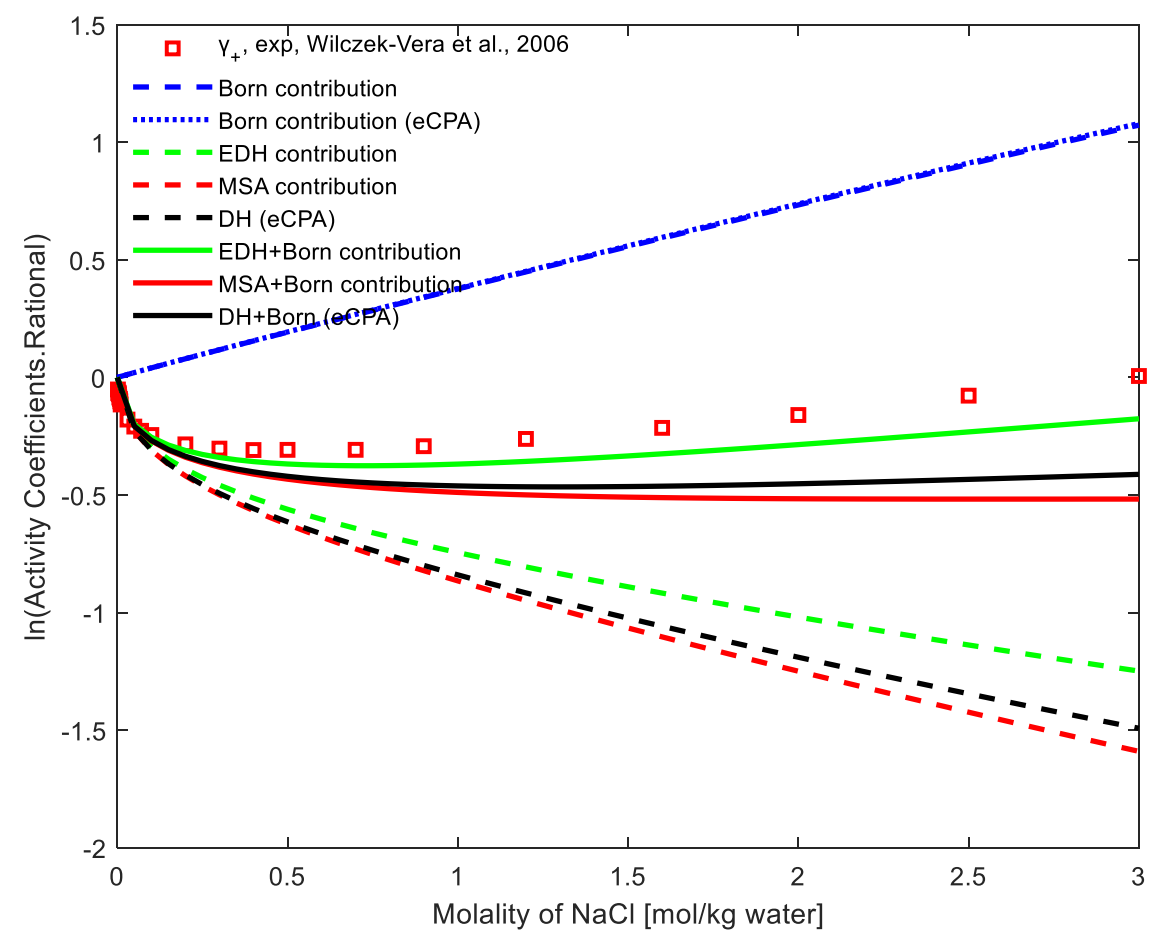

a. activity coefficients of cation ${ }^{10}$ 


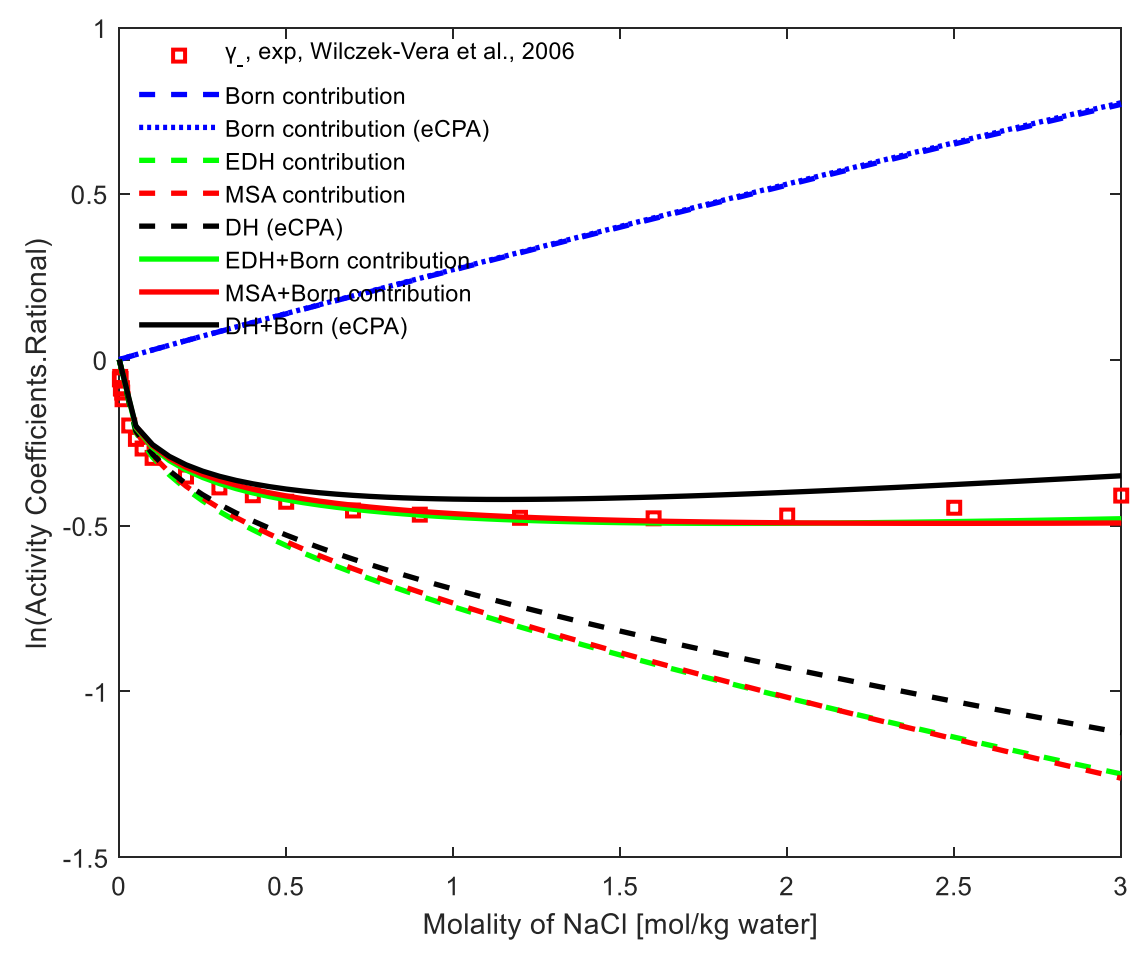

b. activity coefficients of anion ${ }^{10}$

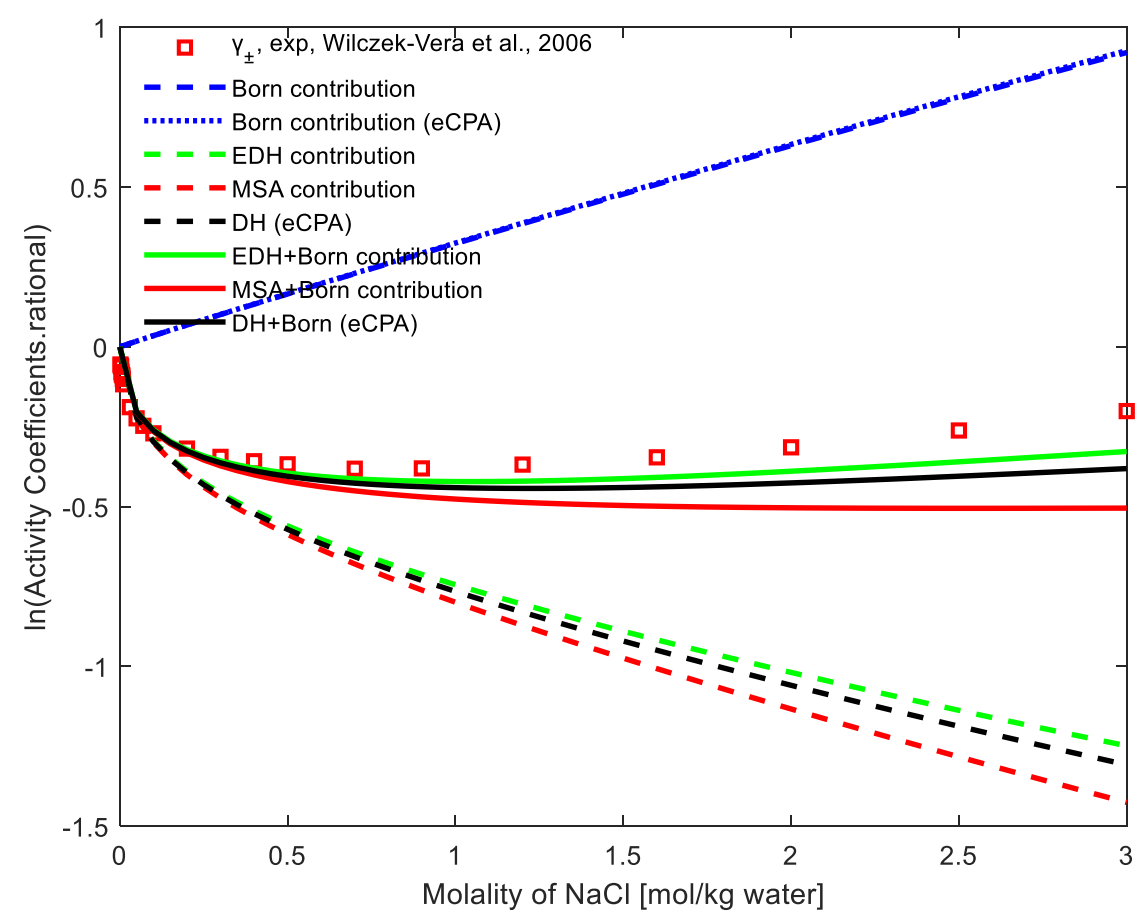

c. mean ionic activity coefficients ${ }^{10}$

Figure 1. II+IW performance of ACI and mean ionic activity coefficients of $\mathrm{NaCl}$ in aqueous solution. 


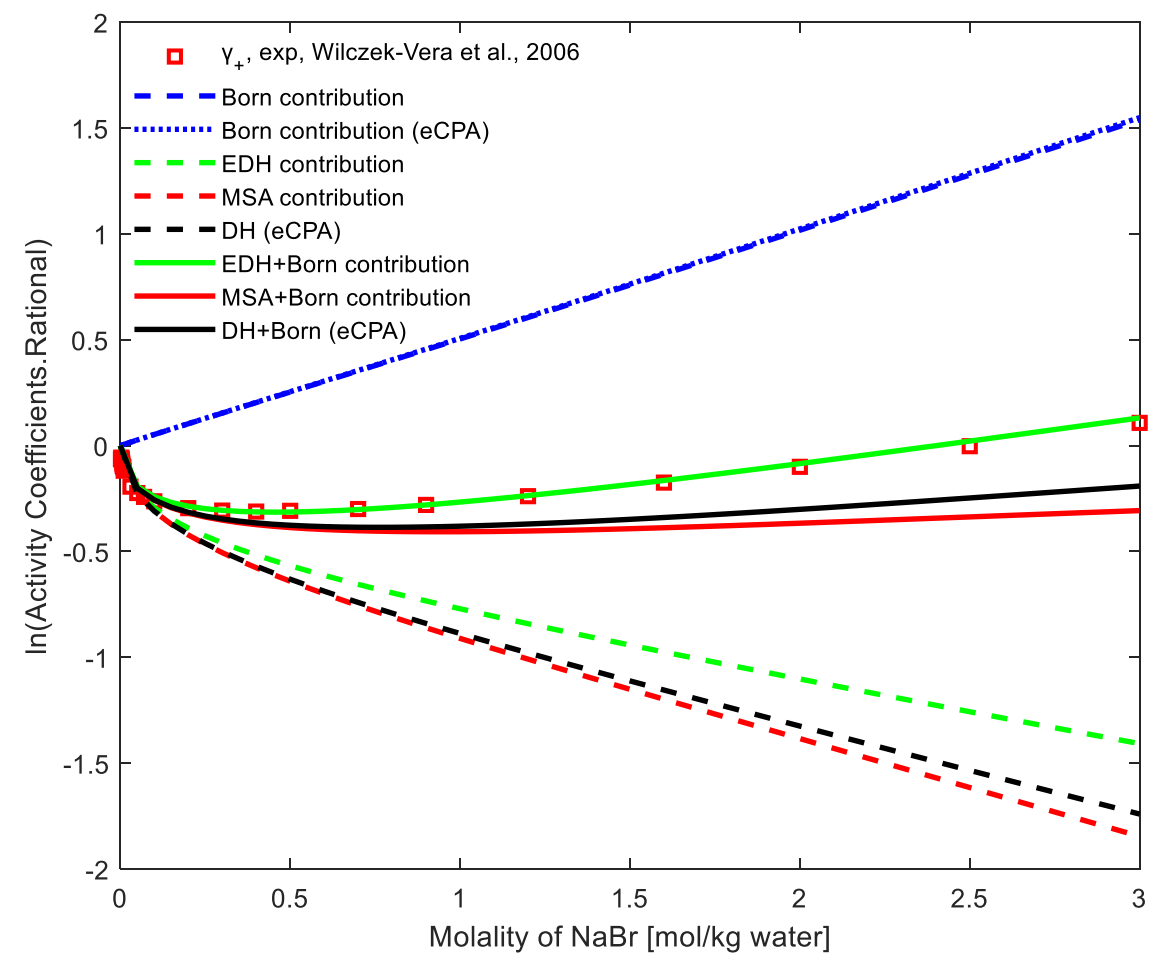

a. activity coefficients of cation ${ }^{10}$

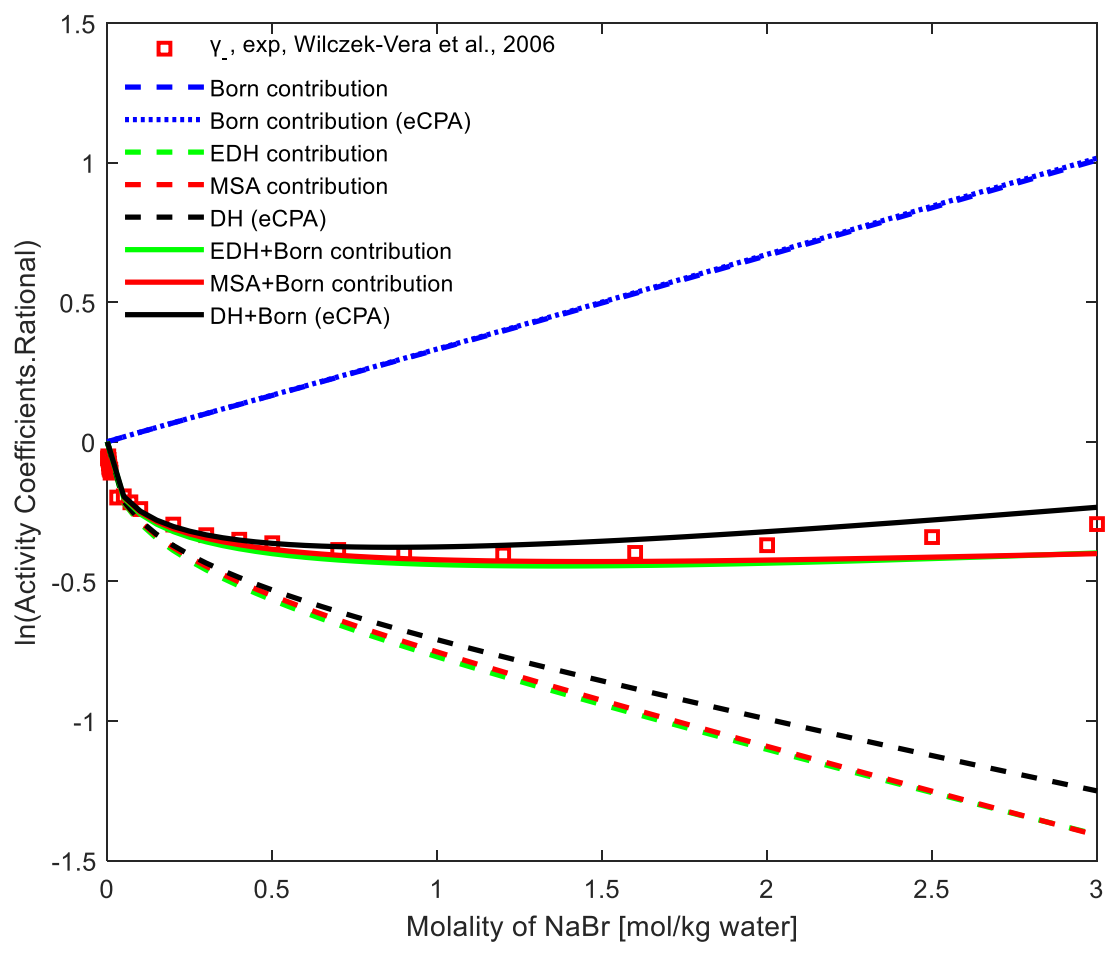

b. activity coefficients of anion ${ }^{10}$ 


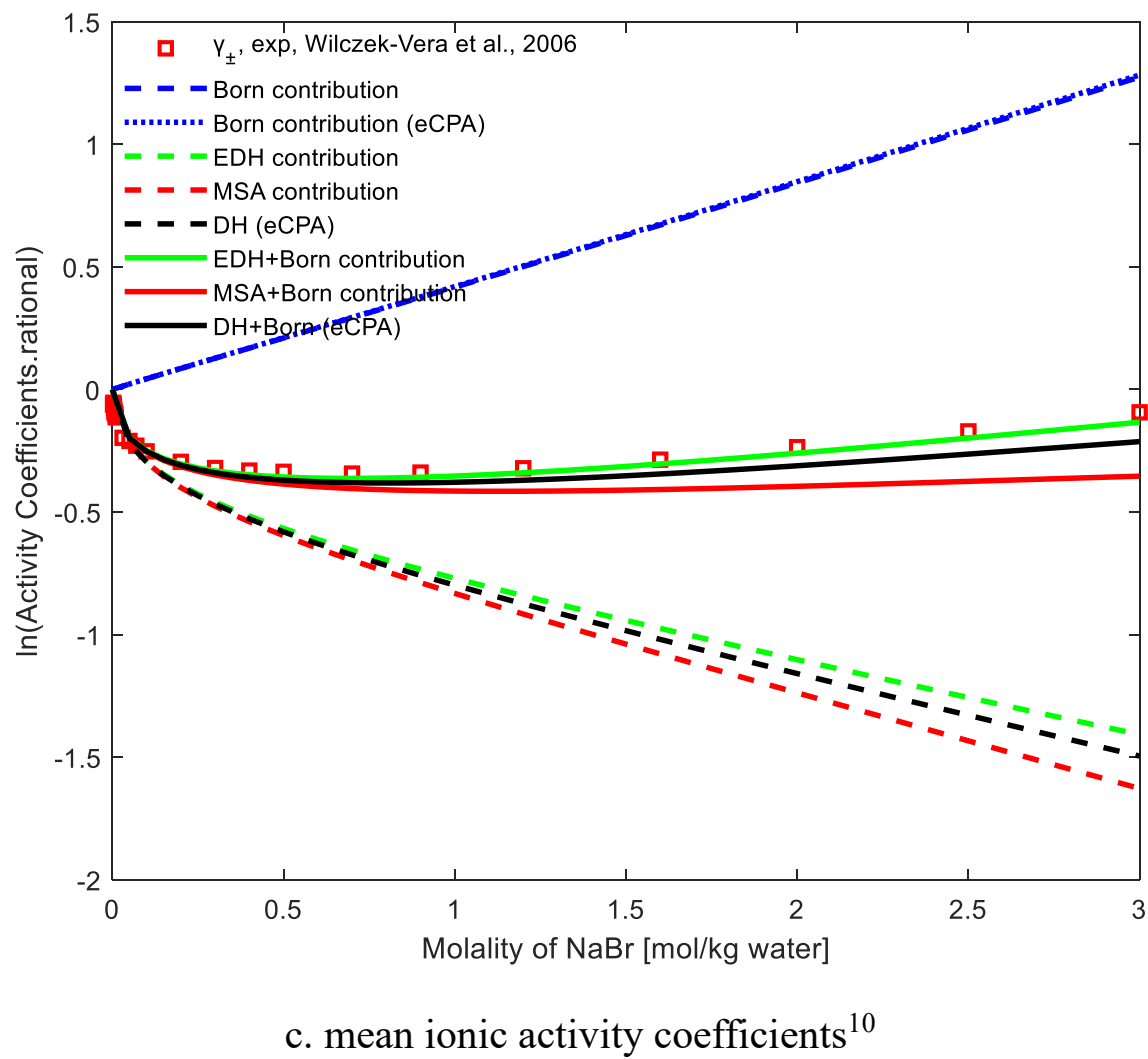

Figure 2. II+IW performance of $\mathrm{ACI}$ and mean ionic activity coefficients of $\mathrm{NaBr}$ in aqueous solution.

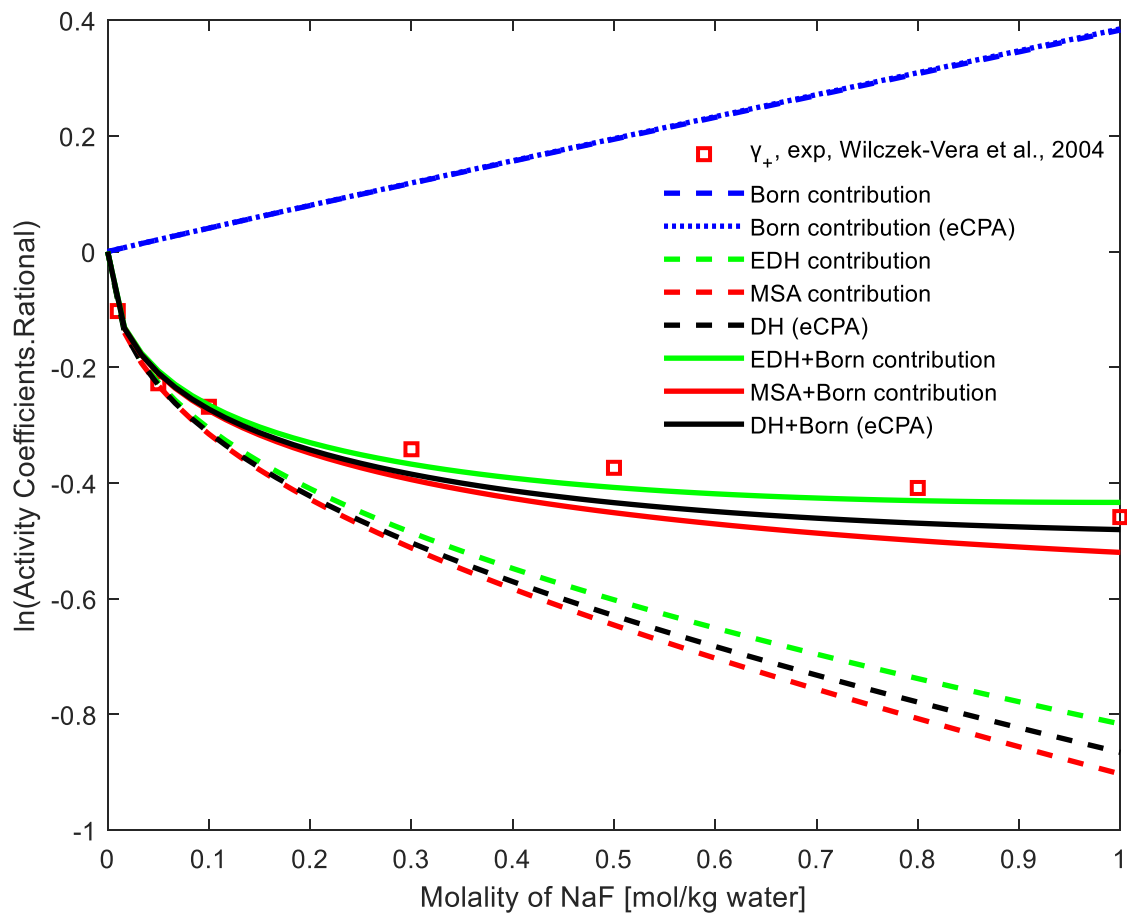

a. activity coefficients of cation ${ }^{9}$ 


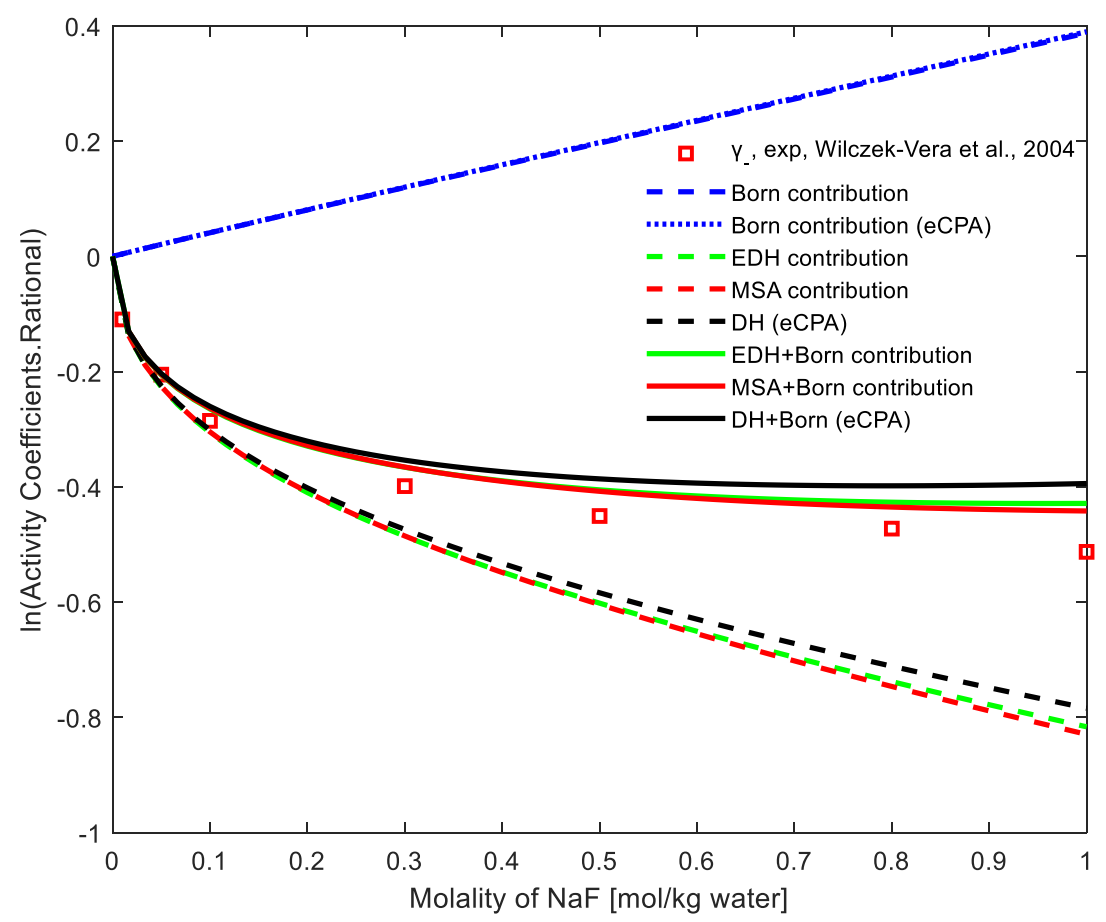

b. activity coefficients of anion ${ }^{9}$

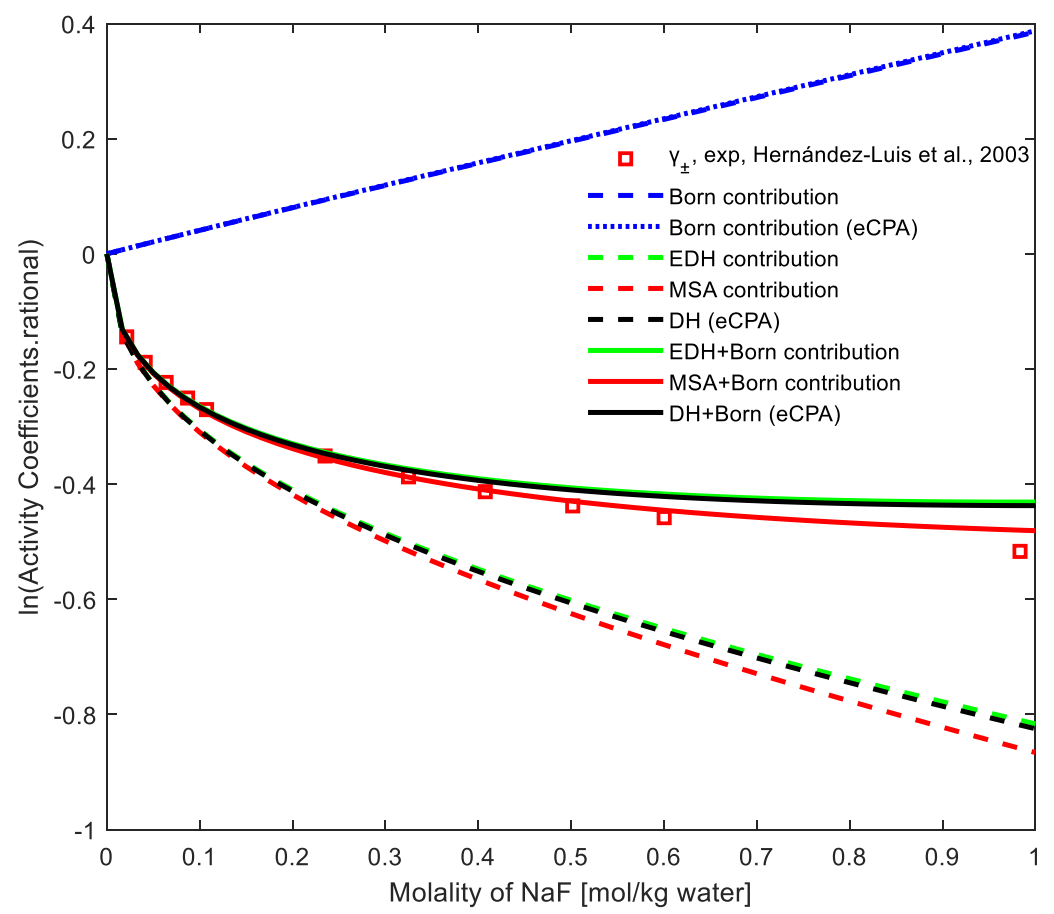

c. mean ionic activity coefficients ${ }^{53}$

Figure 3. II+IW performance of $\mathrm{ACI}$ and mean ionic activity coefficients of $\mathrm{NaF}$ in aqueous solution. 


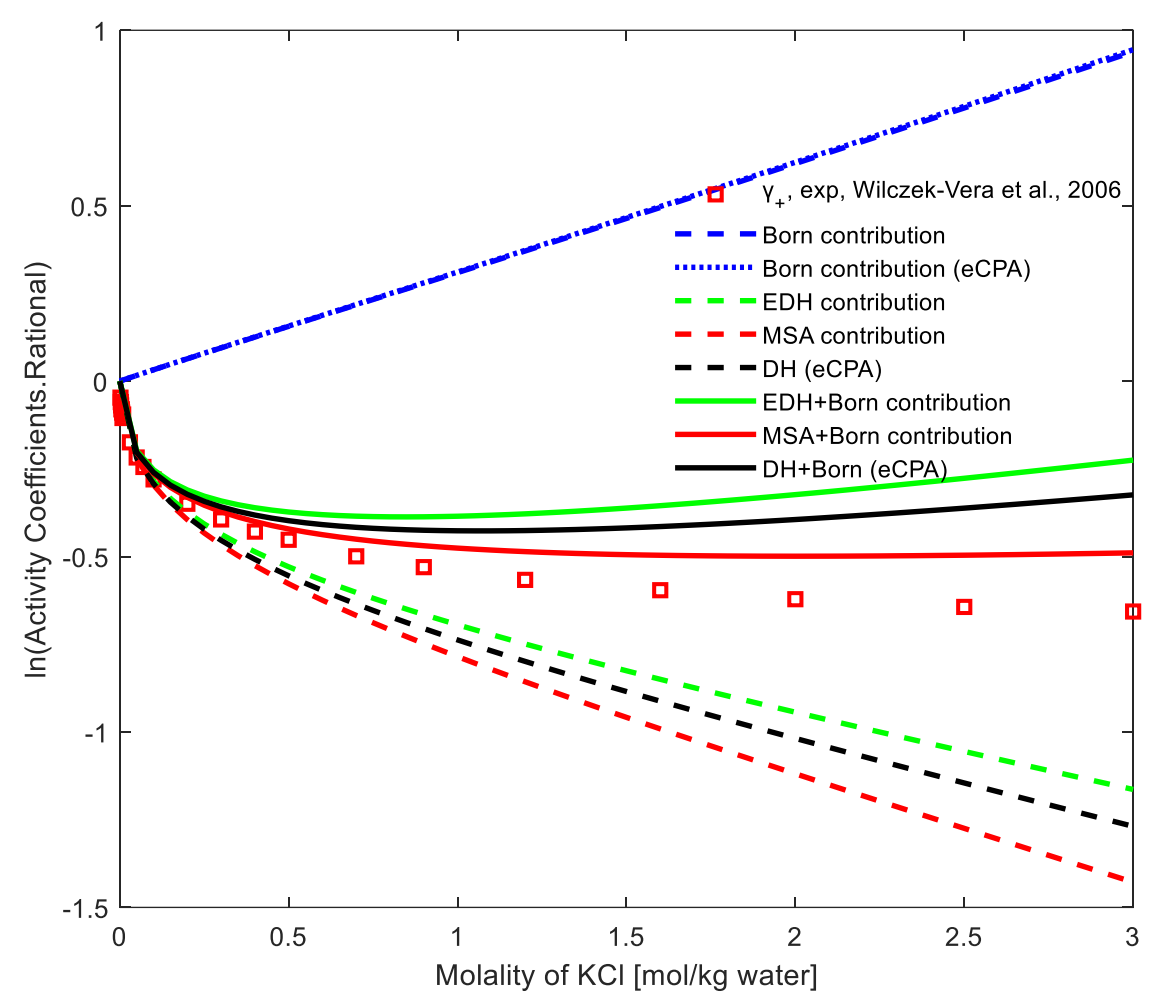

a. activity coefficients of cation ${ }^{10}$

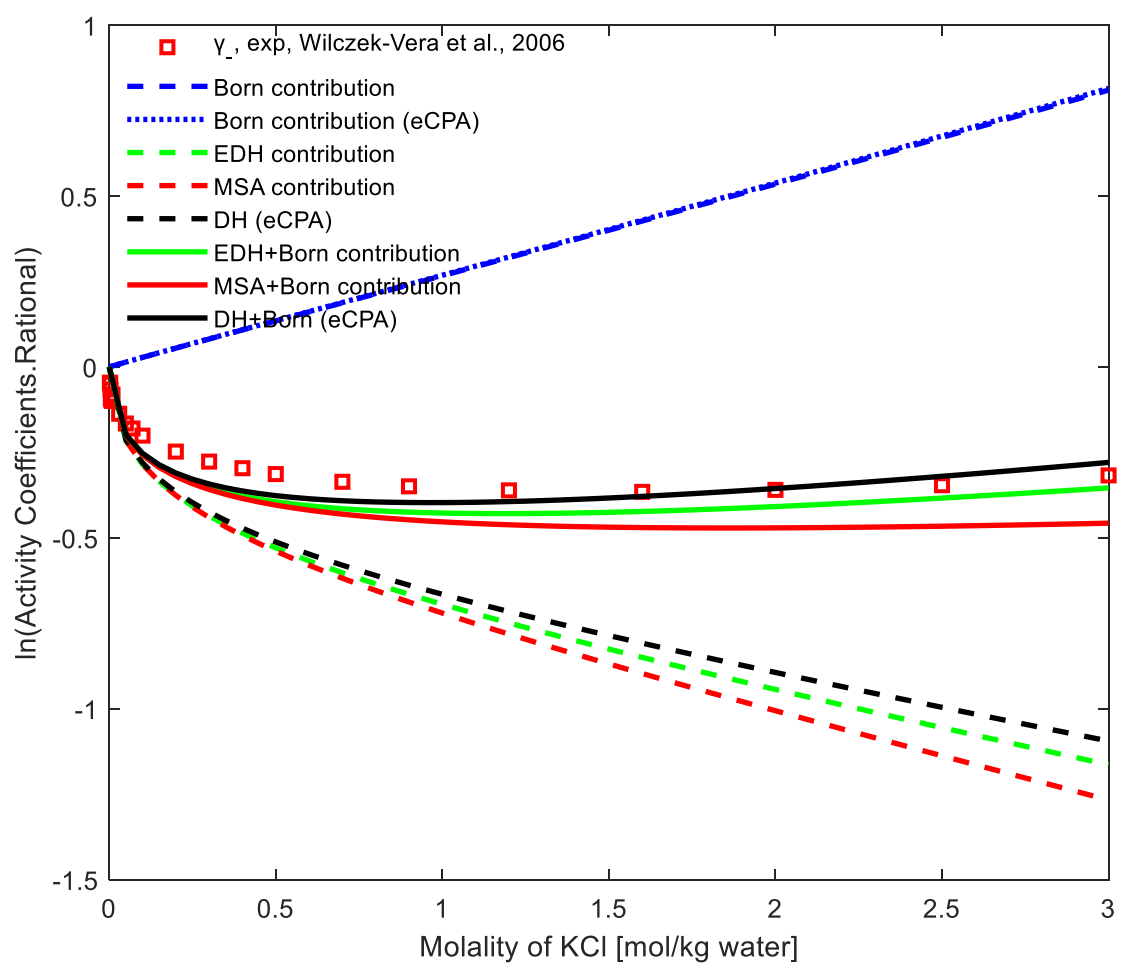

b. activity coefficients of anion ${ }^{10}$ 


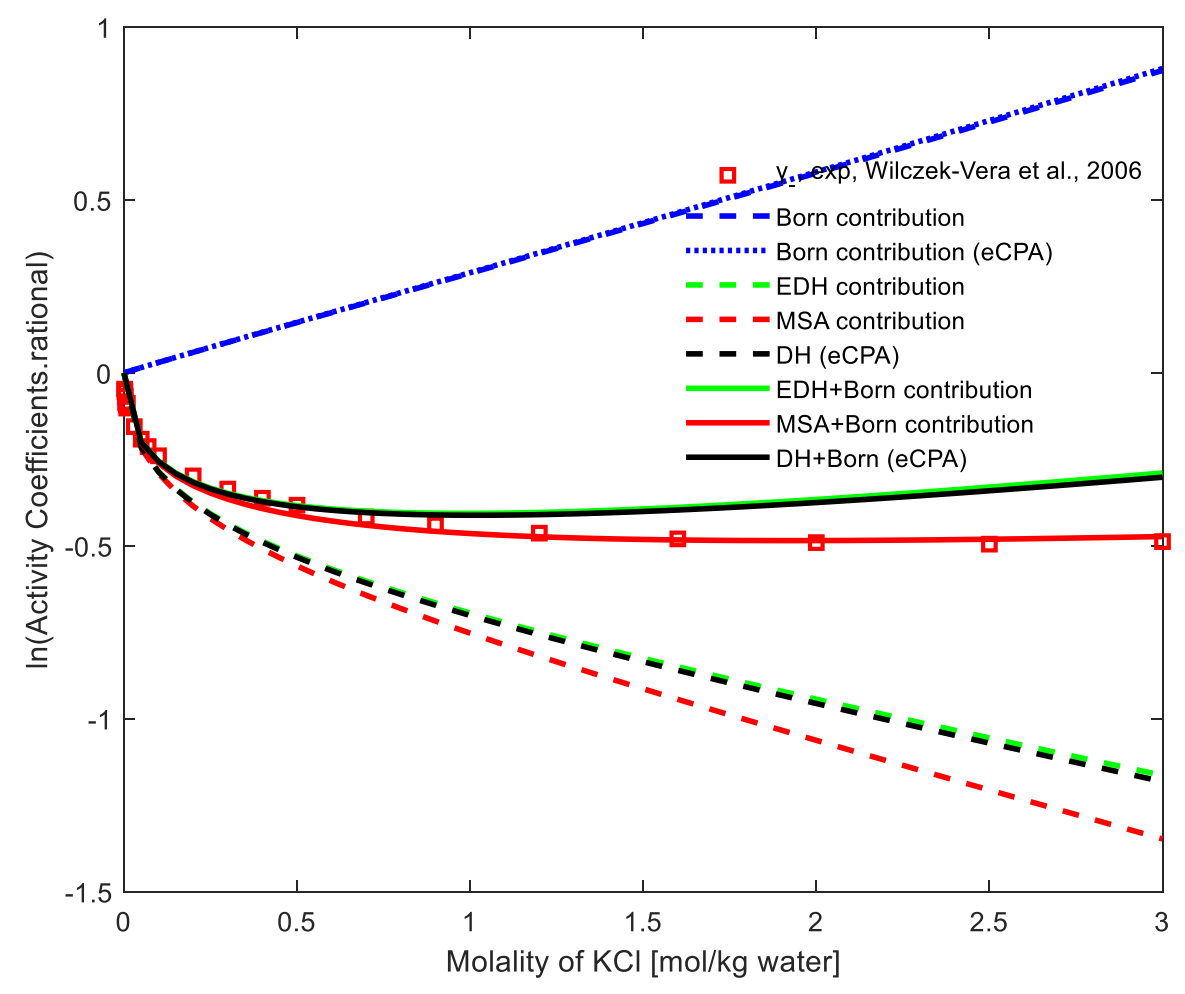

c. mean ionic activity coefficients ${ }^{10}$

Figure 4. II+IW performance of $\mathrm{ACI}$ and mean ionic activity coefficients of $\mathrm{KCl}$ in aqueous solution.

As shown in figures 5 and 6, for 2:1 electrolyte systems, the EDH+Born performance is better than the other two models for activity coefficients of cation. For activity coefficients of $\mathrm{Cl}^{-}$and both for $\mathrm{CaCl}_{2}$ and $\mathrm{MgCl}_{2}$, the performance of $\mathrm{EDH}+\mathrm{Born}$ is worse than MSA+Born and II+IW (e-CPA). Still, the differences between the various modeling approaches are rather small (both for single ion and even for mean ionic activity coefficients) and the same qualitative conclusions as for 1:1 electrolytes also apply here e.g. about the large opposing values and trends of Born and DH/MSA terms. As DH (e-CPA) and EDH use essentially the same ion-ion term, the 
differences between these two approaches illustrate the effect of different parameters, like values of ion radius, on the results. We should point out that, the activity coefficients of $\mathrm{Cl}^{-}$back calculated from experimental activity coefficients of $\mathrm{Ca}^{2+}$ show large deviations from the experimental activity coefficients of $\mathrm{Cl}^{-}$.

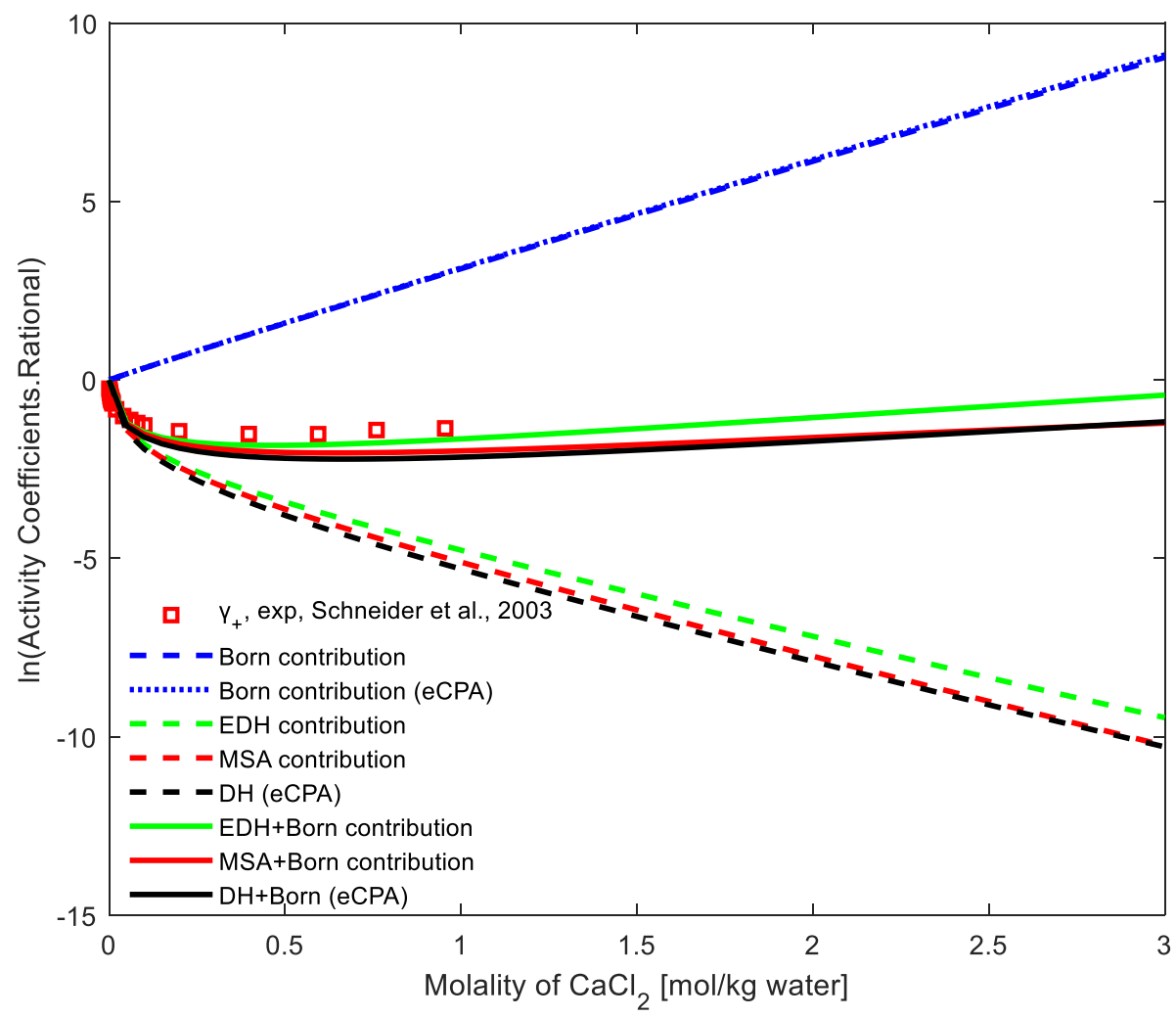

a. activity coefficients of cation ${ }^{54}$ 


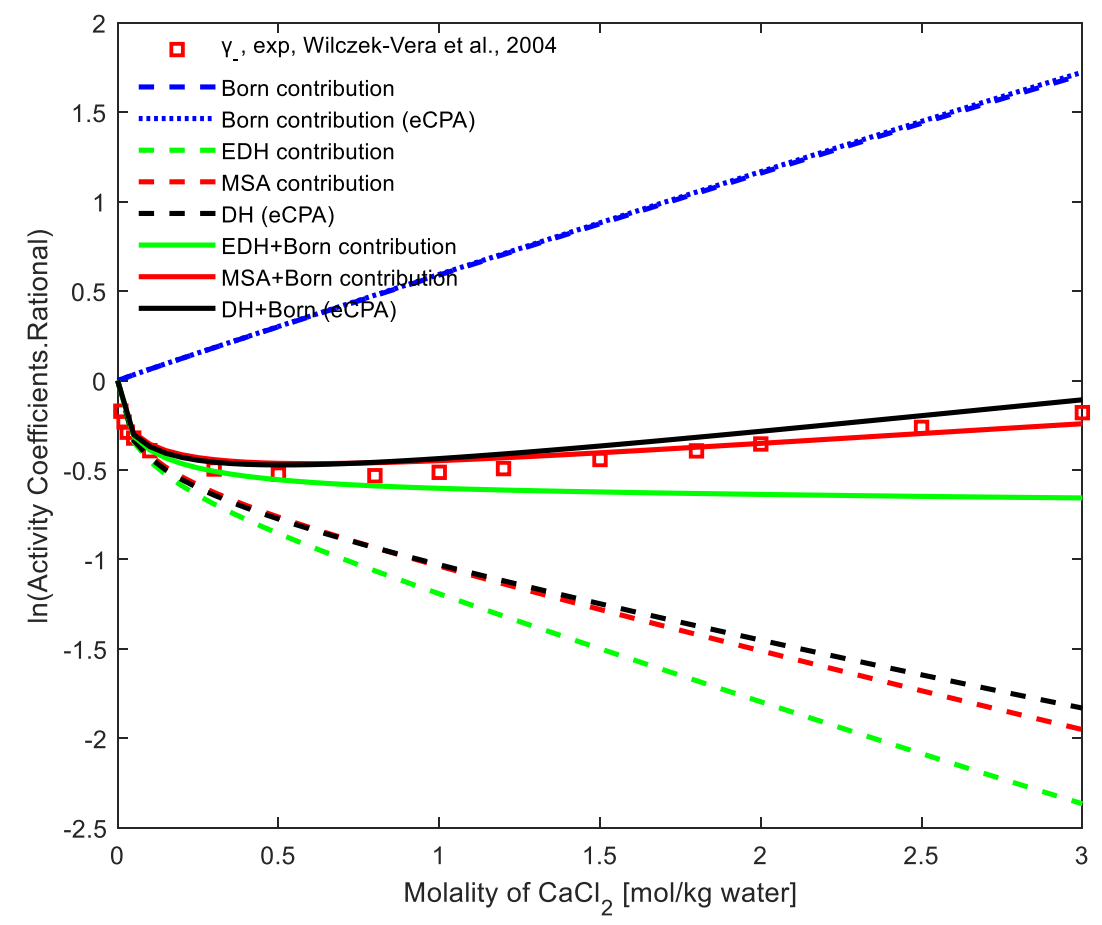

b. activity coefficients of anion 9

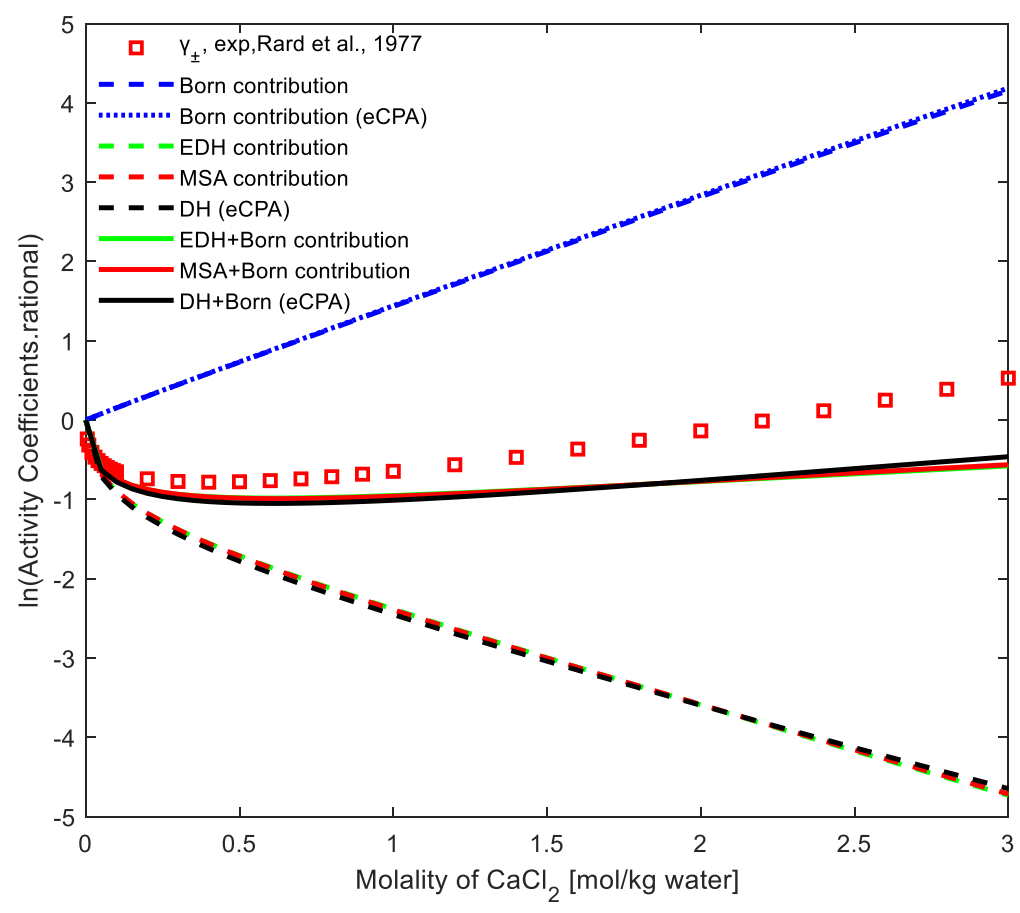

c. mean ionic activity coefficients ${ }^{55}$

Figure 5. II+IW performance of ACI and mean ionic activity coefficients of $\mathrm{CaCl}_{2}$ in aqueous solution. 


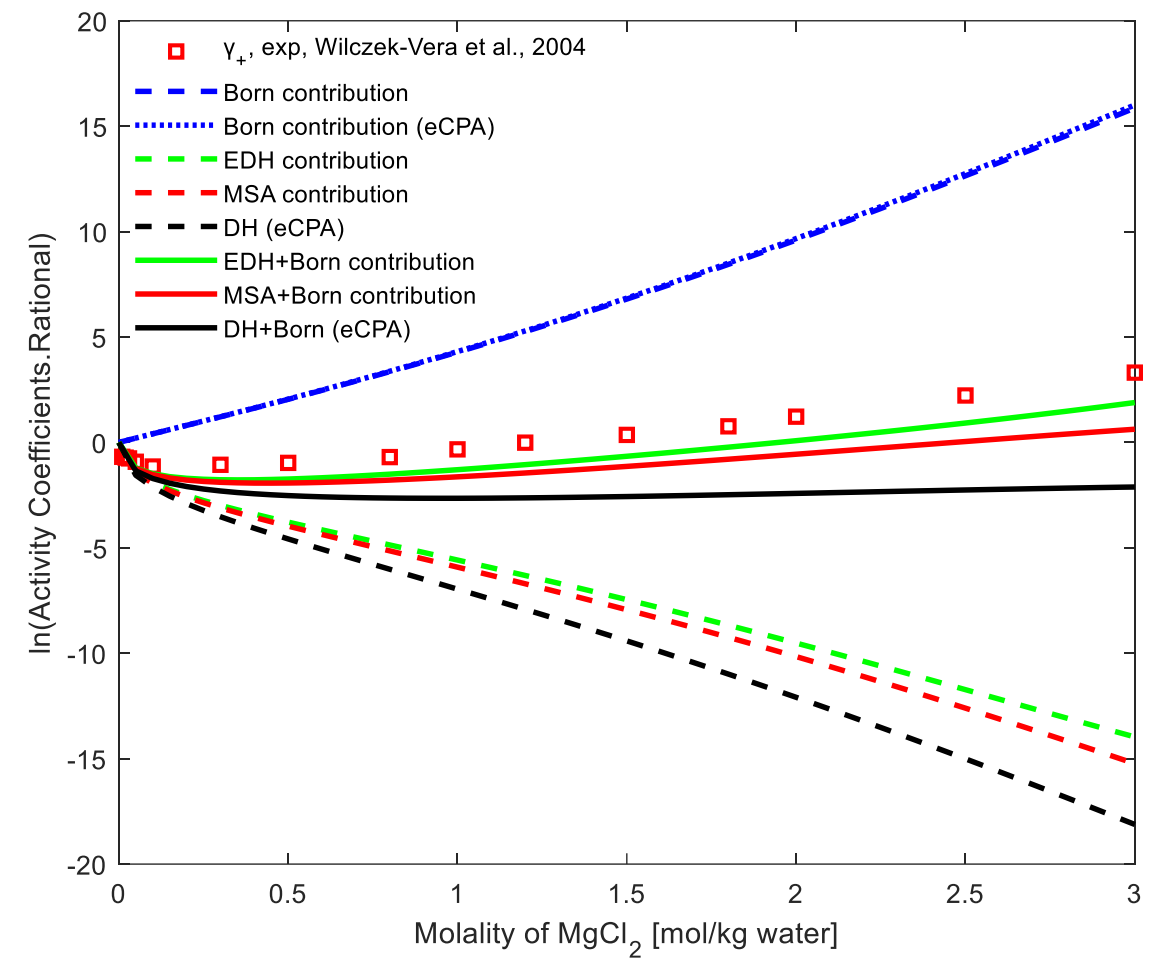

a. activity coefficients of cation ${ }^{9}$

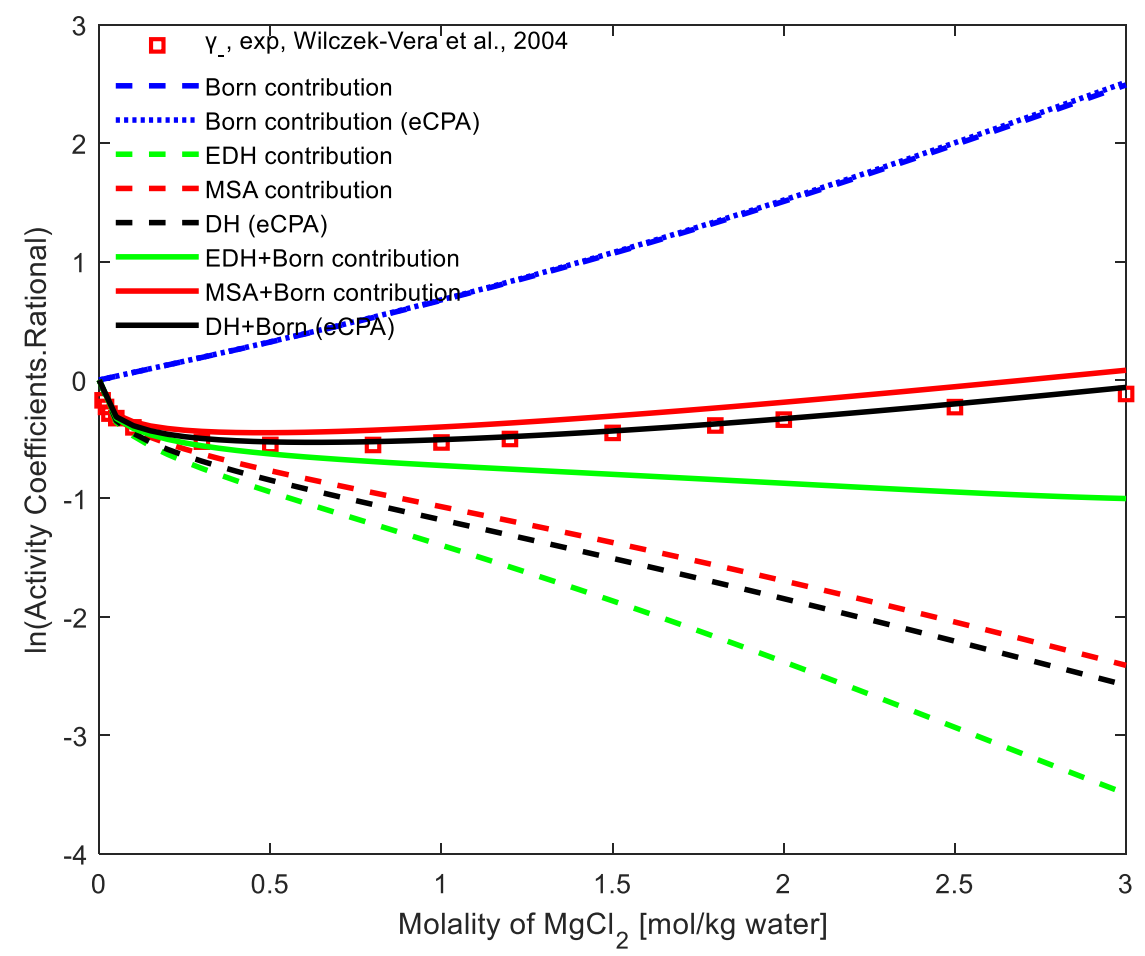

b. activity coefficients of anion ${ }^{9}$ 


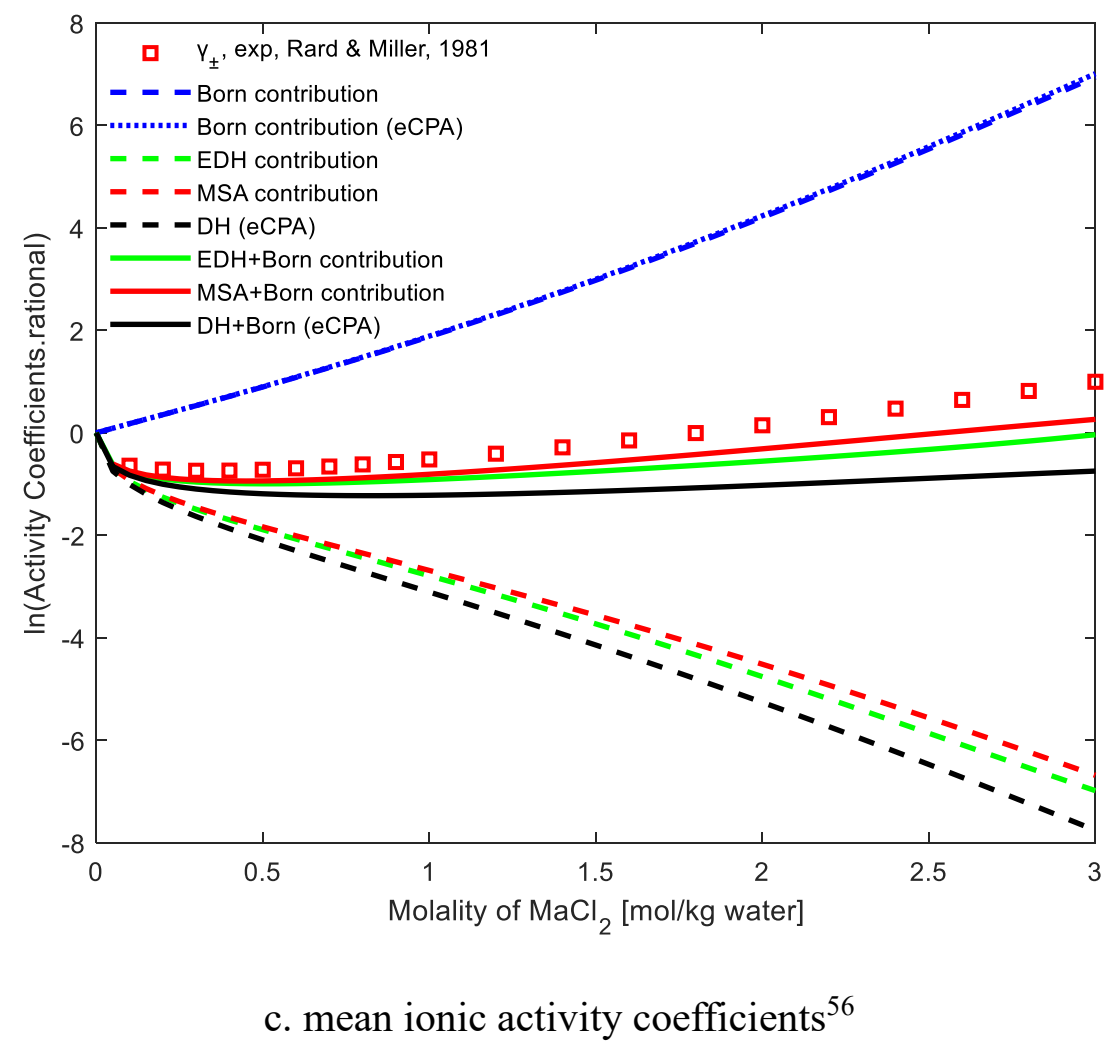

Figure 6. II+IW performance of ACI and mean ionic activity coefficients of $\mathrm{MgCl}_{2}$ in aqueous solution.

For 1:2 electrolyte systems (Figures 7 and 8), the performance of MSA+Born is much better that that of the other two models. The II+IW version from e-CPA performs overall worse than the other two models. Clearly in this case we see a larger difference between the modelling approaches. For the DH (e-CPA) and EDH models, it appears that the ionic term alone is rather close to single ion data, resulting (when the Born term is added) to significant deviations. The differences between the models, and thus the deviations from experimental data, are smaller for mean ionic activity coefficients compared to ACI. 


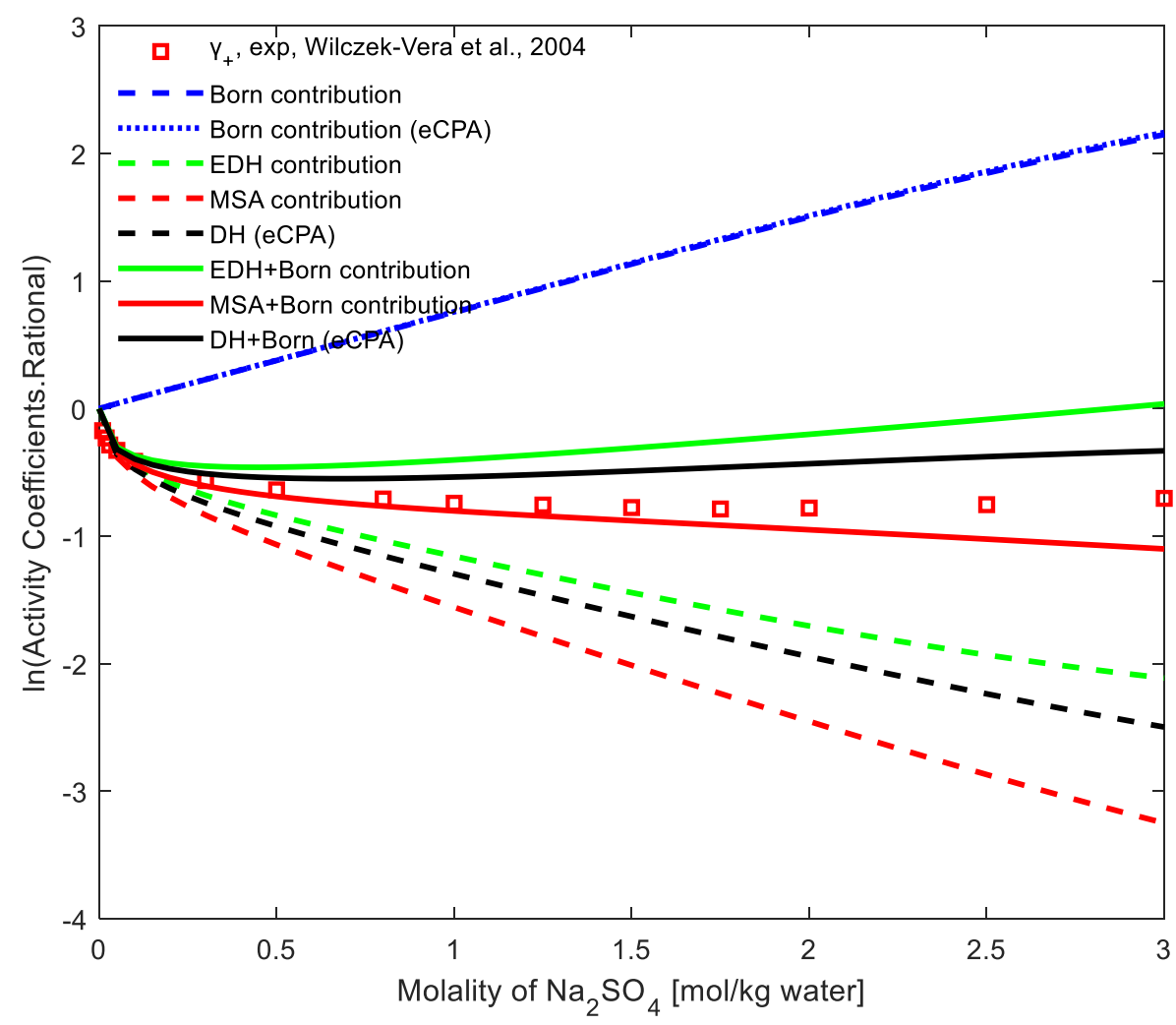

a. activity coefficients of cation ${ }^{9}$

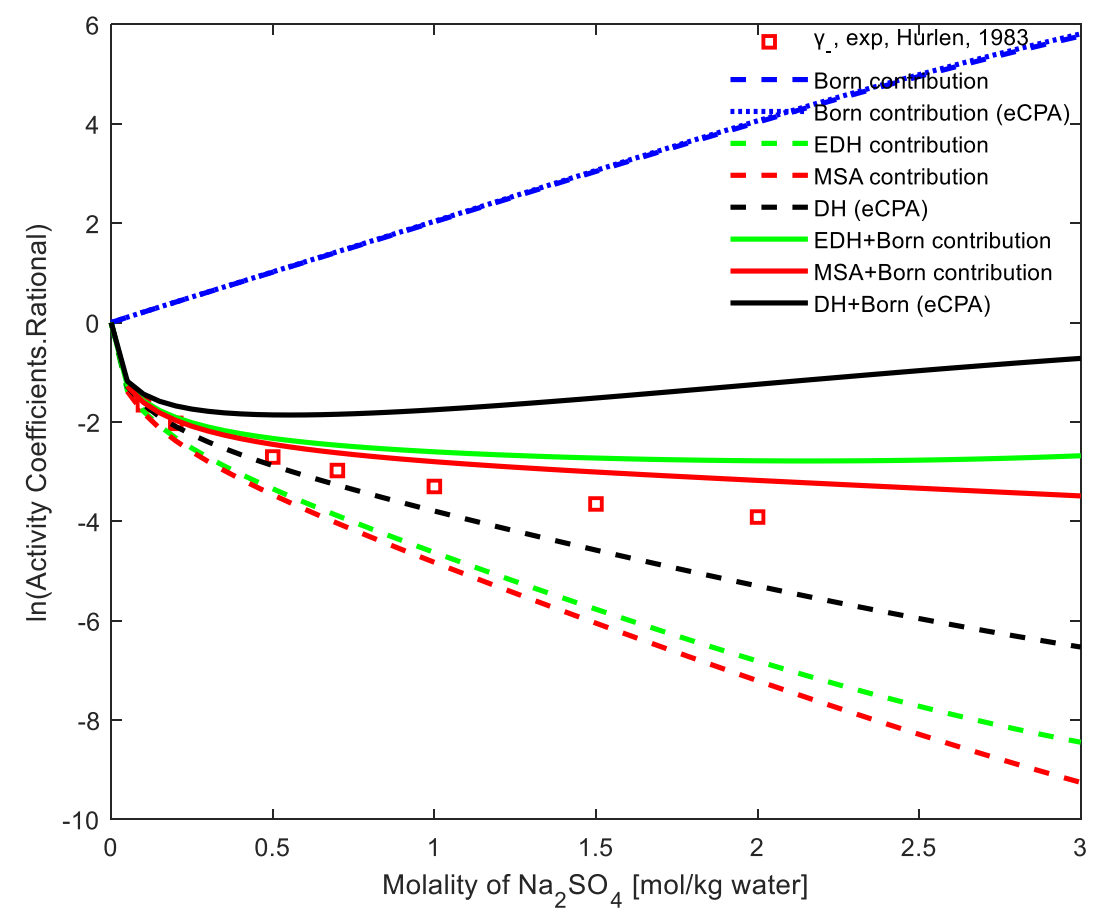

b. activity coefficients of anion ${ }^{57}$ 


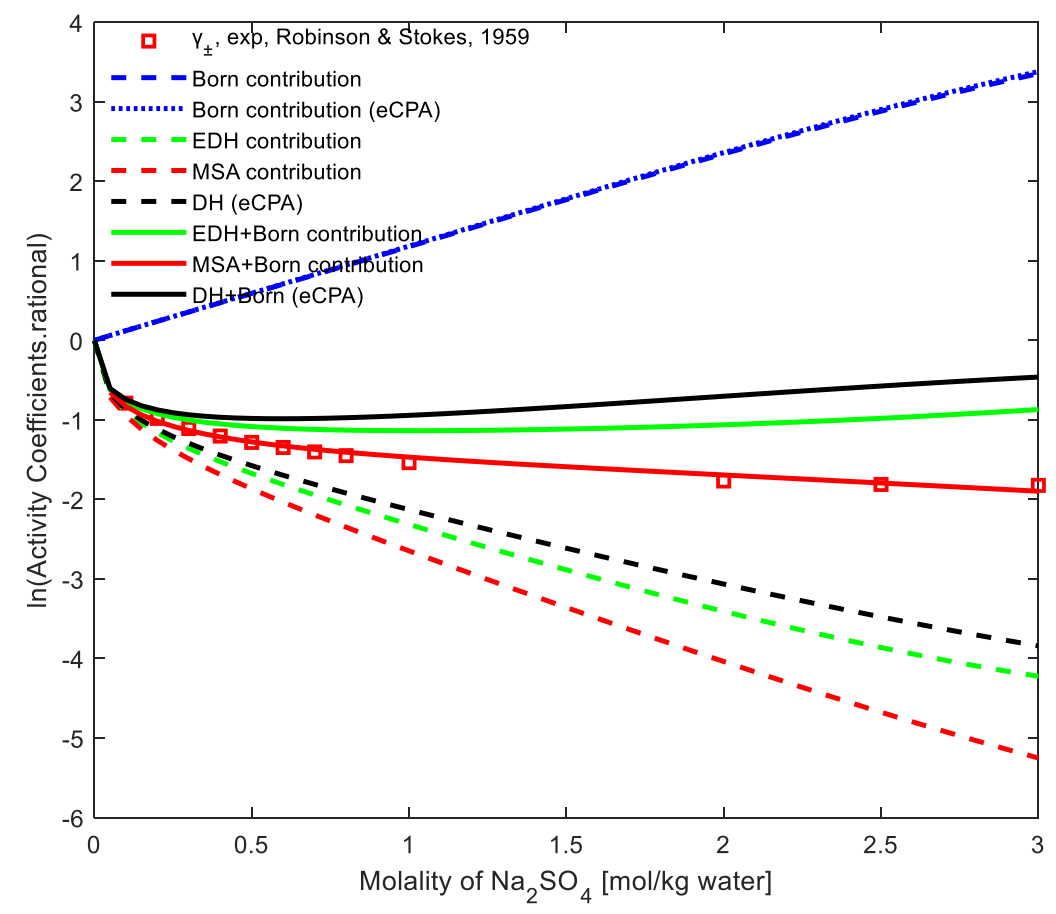

c. mean ionic activity coefficients ${ }^{36}$

Figure 7. II+IW performance of ACI and mean ionic activity coefficients of $\mathrm{Na}_{2} \mathrm{SO}_{4}$ in aqueous solution.

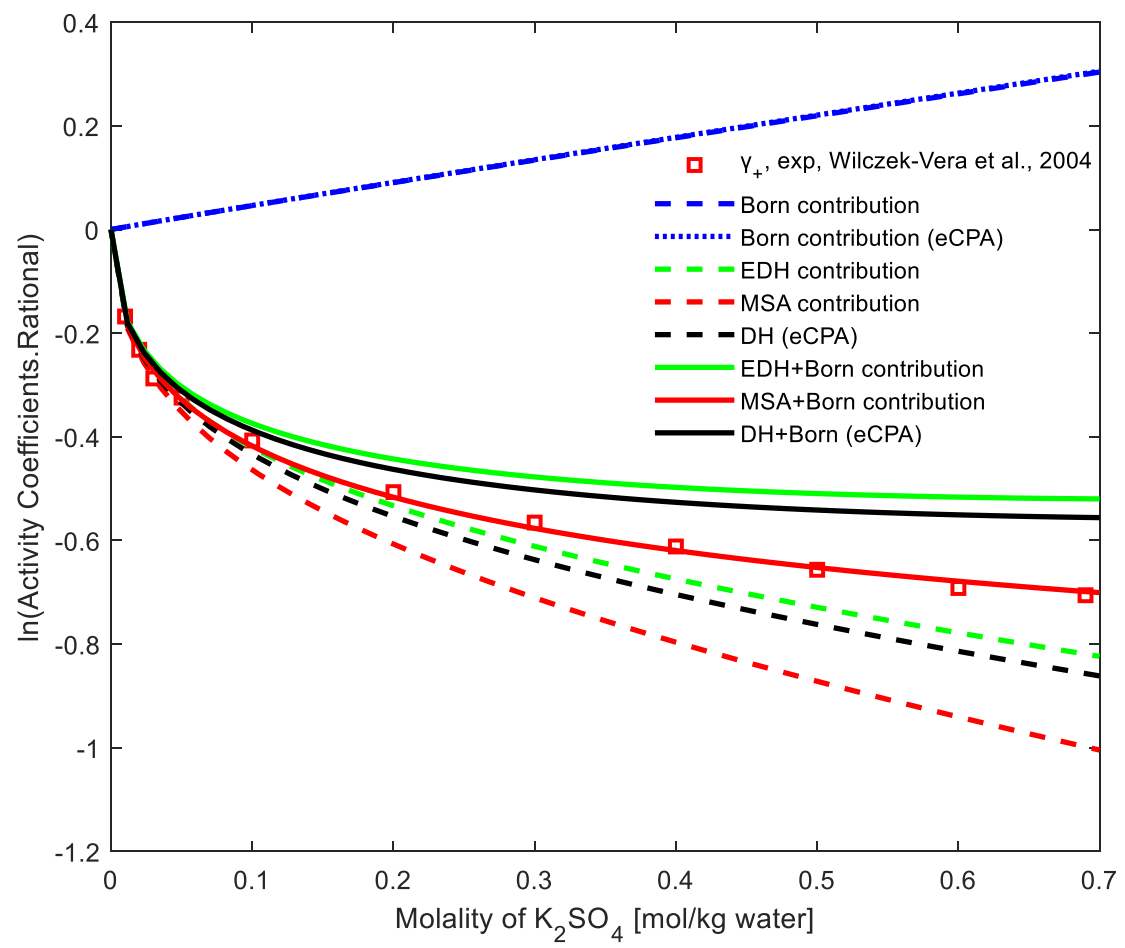

a. activity coefficients of cation ${ }^{9}$ 


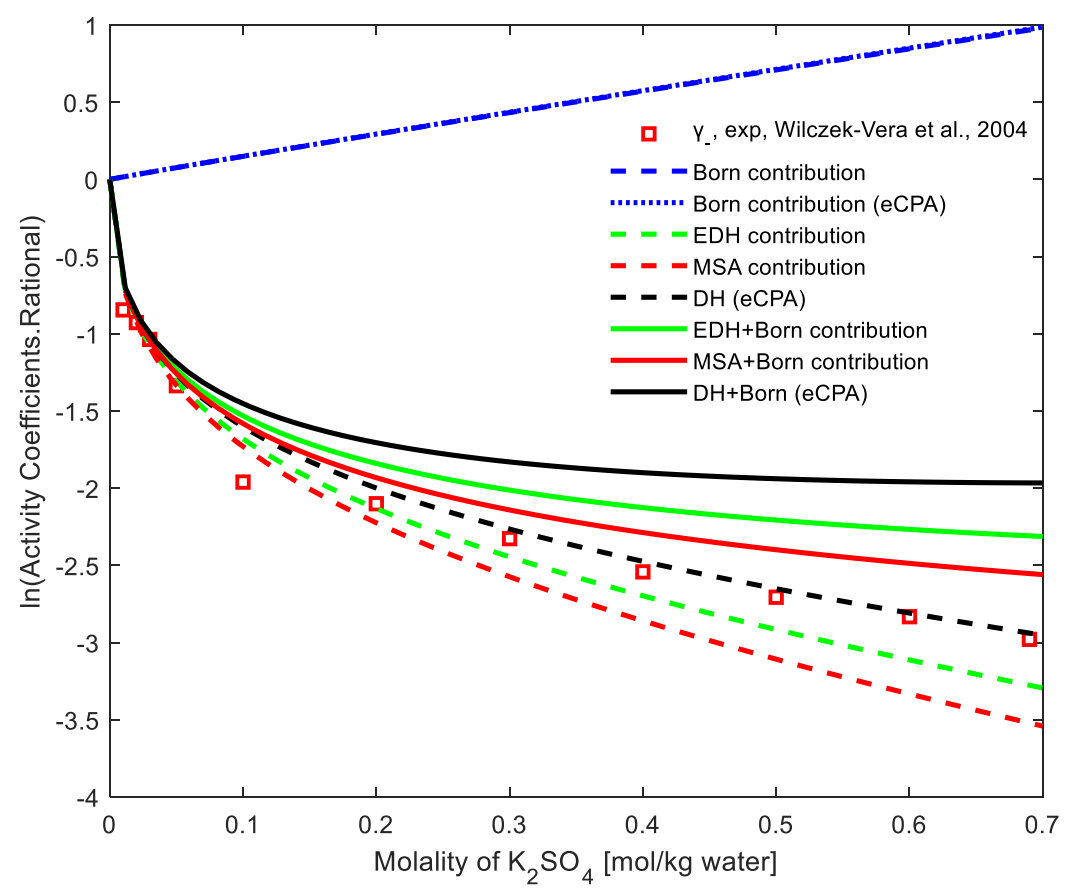

b. activity coefficients of anion ${ }^{9}$

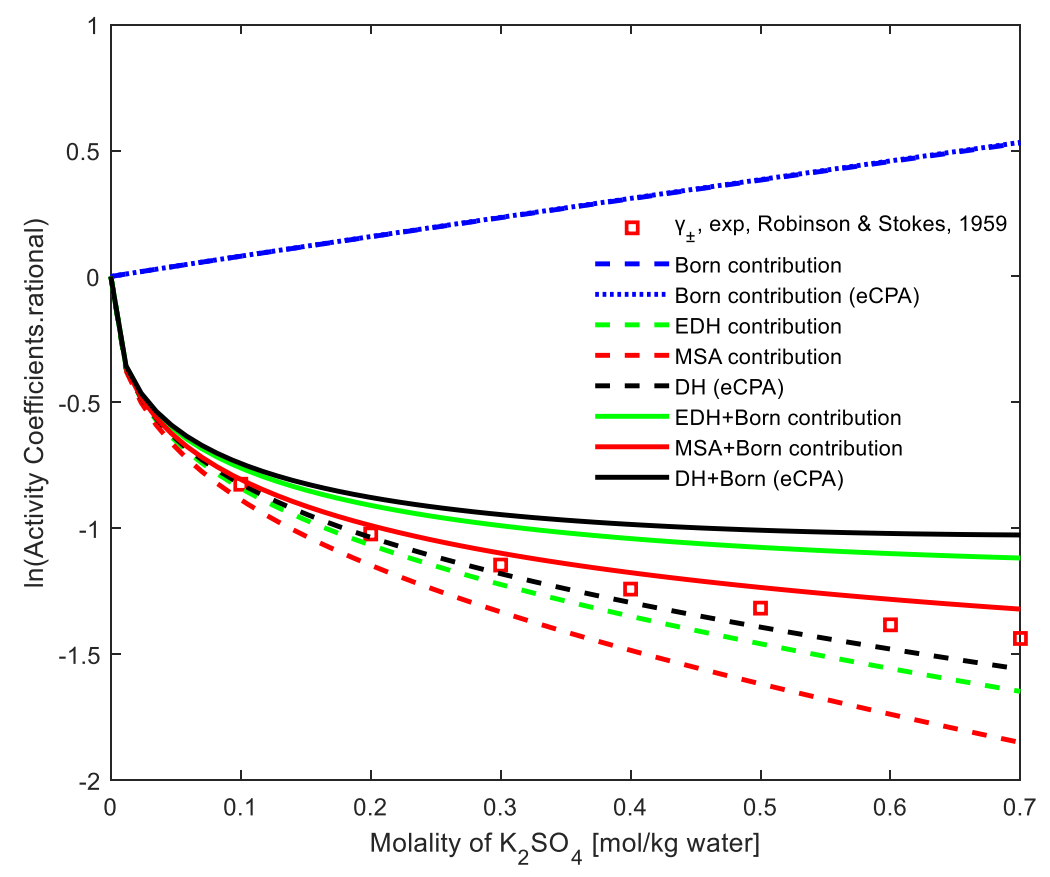

c. mean ionic activity coefficients ${ }^{36}$

Figure 8. II+IW performance of ACI and mean ionic activity coefficients of $\mathrm{K}_{2} \mathrm{SO}_{4}$ in aqueous solution. 
Finally, from Figure 9, it can be seen that the II+IW theory performance is good for the ACI in aqueous $\mathrm{LaCl}_{3}$ solutions. The II and IW contributions of $\mathrm{La}^{3+}$ vary in a wider range than those of $\mathrm{Cl}^{-}$due to their charge ratio, but still the performance is very good. We see a slightly different qualitative trend for II+IW (e-CPA) contributions, but still the performance of all models is acceptable and for both the single ion and mean ionic activity coefficients.

From Table 2 and Figures 1-9, it can be seen that all three models can for many systems predict very satisfactorily the trend of ACI and mean ionic activity coefficients.

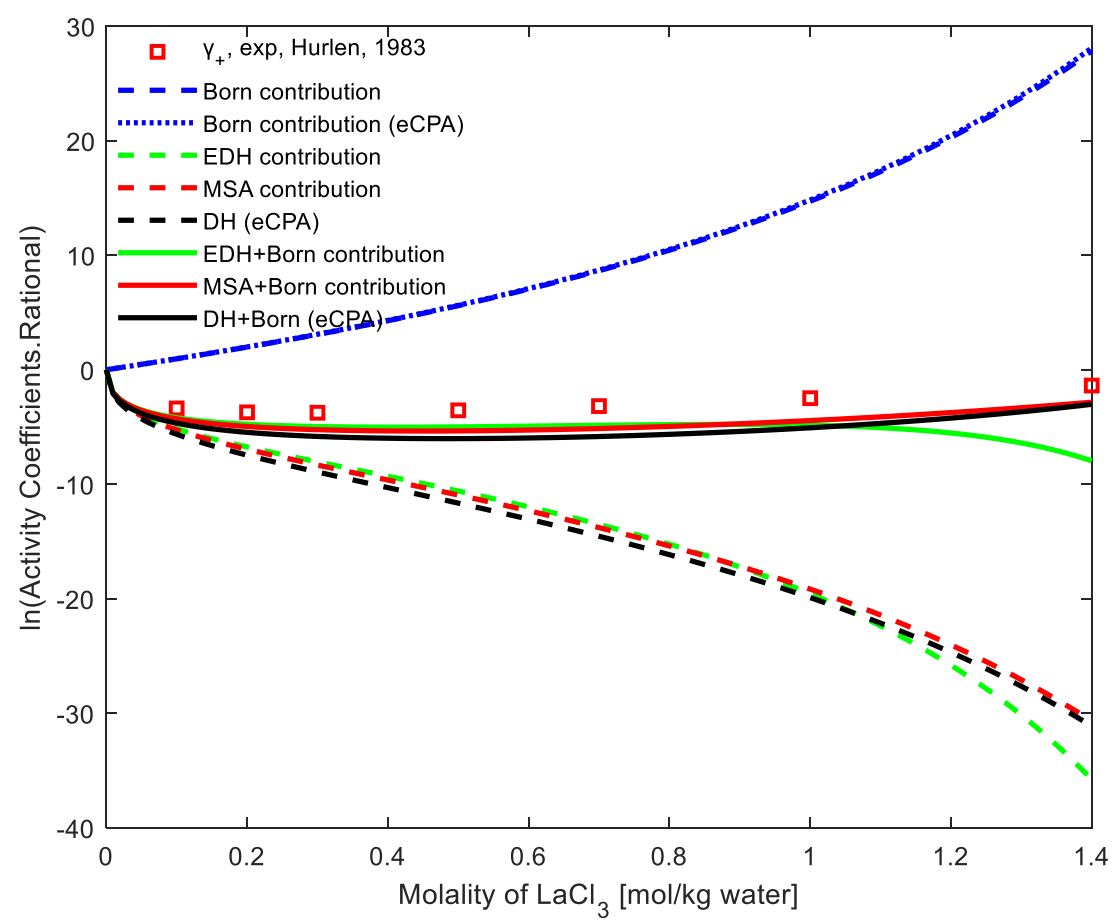

a. activity coefficients of cation ${ }^{58}$ 


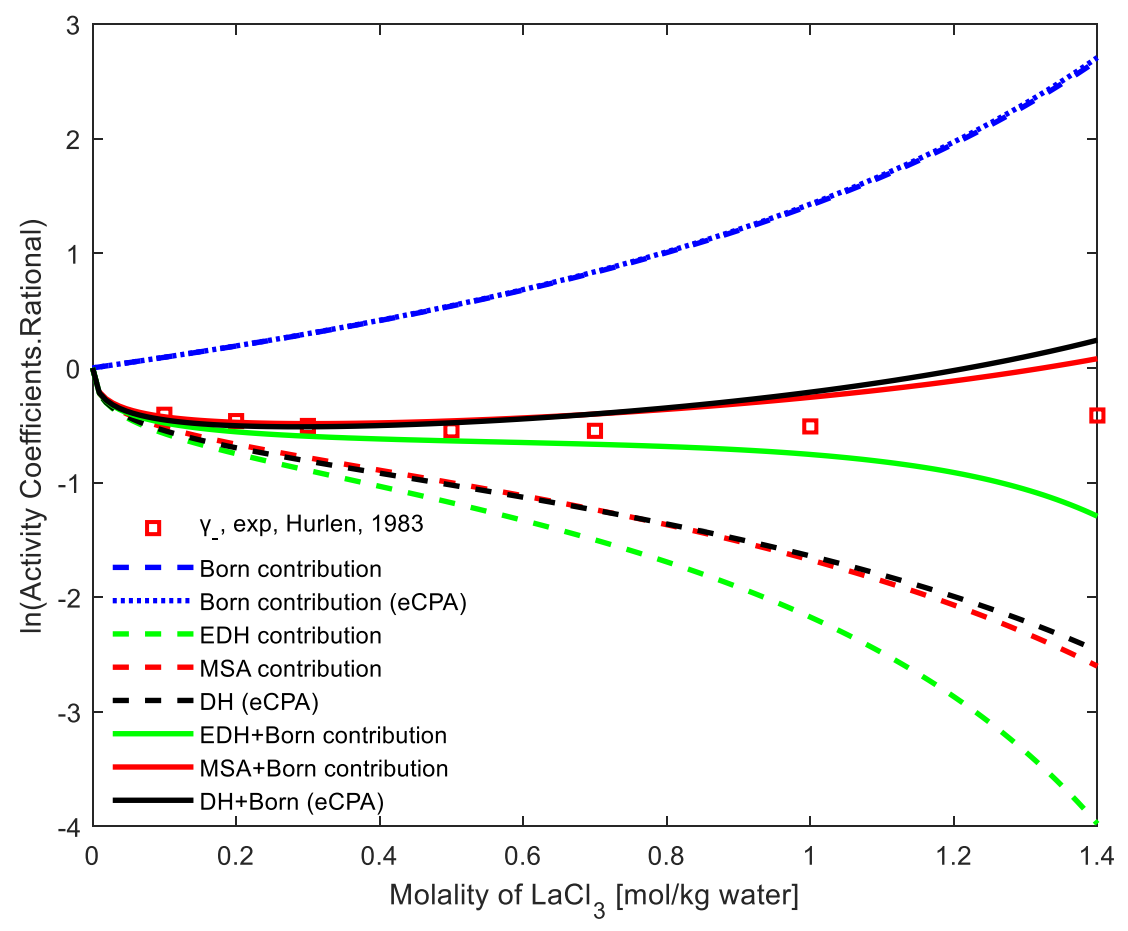

b. activity coefficients of anion ${ }^{58}$

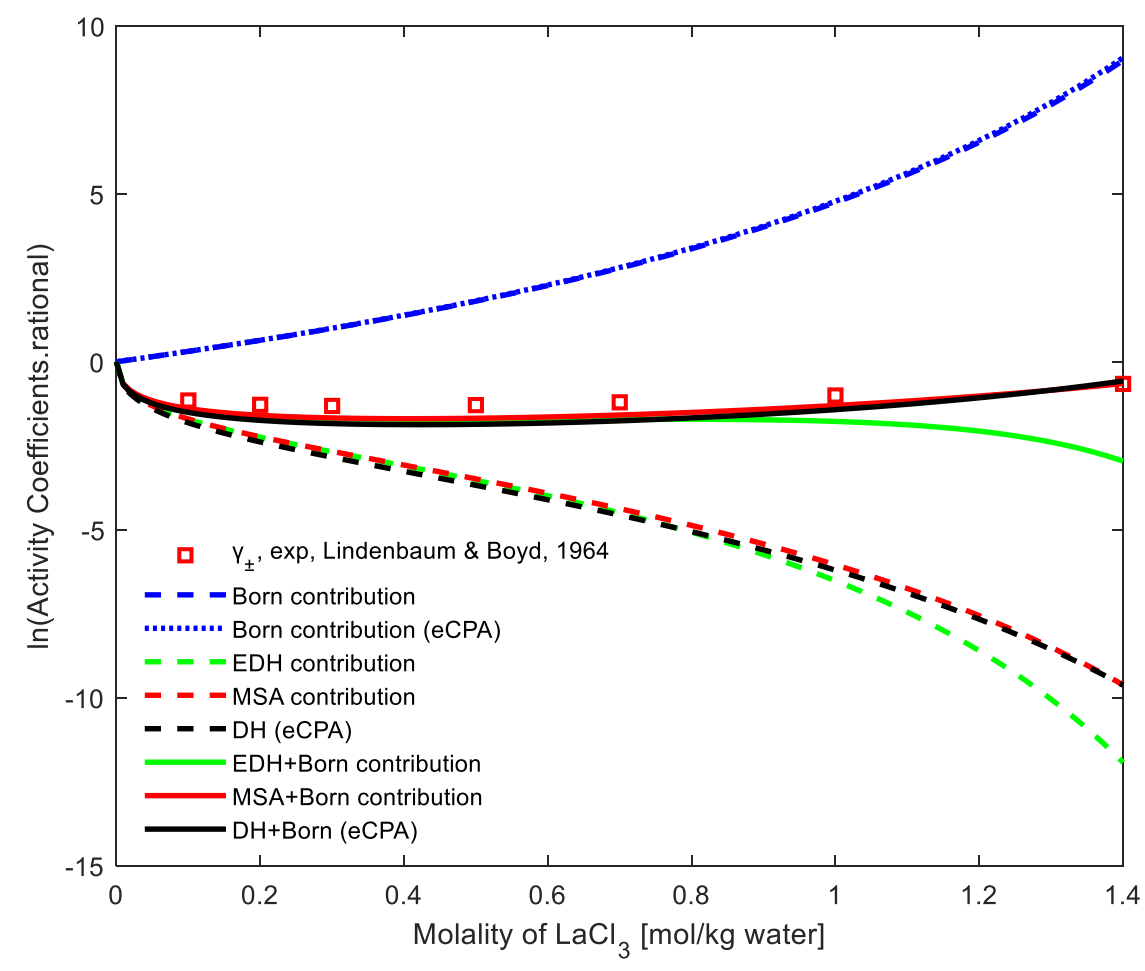

c. mean ionic activity coefficients ${ }^{58}$

Figure 9. II+IW performance of ACI and mean ionic activity coefficients of $\mathrm{LaCl}_{3}$ in aqueous solution. 
In all cases, the total (individual ion and mean ionic) activity coefficient appear to result as a balance between two opposing terms of comparably similar magnitudes, a positive contribution from the Born theory and a negative one from EDH or MSA. It appears, thus, that both terms (II and IW) are of equal importance in the representation of electrolyte solutions. This conclusion is in agreement with the studies by Boda, Valiskó, Shilov and Lyashchenko ${ }^{16-17,41}$.

It can be seen from Table 2 that for activity coefficients of anions, the best performance for all salts is from $\mathrm{DH}+\mathrm{Born}$ (e-CPA). For mean ionic activity coefficients, the best performance for all salts comes from MSA+Born. From the results of $\mathrm{EDH}+$ Born, the deviations for 2:1 electrolytes, 1:2 electrolytes, and 3:1 electrolytes, are mainly for the multivalent ions. This may be due to the limitation of Born equation for multivalent ions. Still, overall, the differences between the various modeling approaches are rather small.

As mentioned above, in MSA and $\mathrm{EDH}$, the average radius is used instead of individual radii for cation and anion. This difference may be the main reason of the slightly different performance of these models. It should be noted that although no adjustable parameters were used in the II+IW calculations, good and qualitative results are obtained.

\subsection{Results using the e-CPA EoS}

In section 5.1, the combination of $\mathrm{DH}+$ Born two terms (from e-CPA) is used as one of 
the three II+IW approaches. In section 5.2, the whole model (i.e. the e-CPA EoS) is used.

As mentioned above, In the II+IW theory calculations (5.1), Pauling radii of ions, experimental liquid density and experimental relative static permittivity of solutions are used in all approaches (including the "DH+Born" of e-CPA). In e-CPA calculations (the whole e-CPA model, 5.2), Marcus radii of ions, EoS calculated liquid density and relative static permittivity of solutions are used. And in the II+IW theory calculations (5.1), composition derivatives of relative static permittivity are not taken into account, while in e-CPA calculations (5.2), these derivatives are considered.

In e-CPA, $F$ is defined as the reduced Helmholtz energy, $\left(\frac{F}{R T}\right)_{V, T, n}$ is "Helmholtz energy divided by $R T$ " of aqueous electrolyte solution, $\left(\frac{F}{R T}\right)_{V_{0}, T, n_{0}}$ is "Helmholtz energy divided by $R T^{\prime}$ of the reference state (which is solvent with no salt as the reference), the residual Helmholtz energy $\Delta F$ is defined as the difference between $\left(\frac{F}{R T}\right)_{V, T, n}$ and $\left(\frac{F}{R T}\right)_{V_{0}, T, n_{0}}:$
$\Delta F=\left(\frac{F}{R T}\right)_{V, T, n}-\left(\frac{F}{R T}\right)_{V_{0}, T, n_{0}}$

The expression for the contribution terms to activity coefficient of ion is given from the equation:

$$
\begin{aligned}
& \ln \gamma_{i}=\left(\frac{\partial \Delta F^{S R K}}{\partial n_{i}}\right)_{V, T, n_{j}}+\left(\frac{\partial \Delta F^{A s s o}}{\partial n_{i}}\right)_{V, T, n_{j}}+\left(\frac{\partial \Delta F^{D H}}{\partial n_{i}}\right)_{\varepsilon_{r, V, T, n_{j}}} \\
& +\left(\frac{\partial \Delta F^{\text {Born }}}{\partial n_{i}}\right)_{\varepsilon_{r, V, T, n_{j}}}+\left(\frac{\partial \Delta F^{D H}}{\partial \varepsilon_{r}}\right)_{V, T, n_{j}}\left(\frac{\partial \varepsilon_{r}}{\partial n_{i}}\right)_{V, T, n_{j}} \\
& +\left(\frac{\partial \Delta F^{\text {Born }}}{\partial \varepsilon_{r}}\right)_{V, T, n_{j}}\left(\frac{\partial \varepsilon_{r}}{\partial n_{i}}\right)_{V, T, n_{j}}-\ln \frac{Z}{Z_{0}}
\end{aligned}
$$

In Eq. (9), $\varepsilon_{r}$ is the relative static permittivity of solvent in electrolyte solutions, $n_{i}$ 
is the mole number of ion $i, Z$ is the compressibility factor of solution, $Z_{0}$ is the compressibility factor of solvent (with no salt).

$\Delta F^{S R K}$ is the $\Delta F$ from Soave-Redlich-Kwong (SRK) contribution. The ions have volume which cannot be ignored, and the volume-exclusion from ions is present, so the SRK contribution is set for this. The term $-\ln \frac{Z}{Z_{0}}$ is related to the compressibility factor, for the difference based on mole numbers of ions and the combination based on mole number of salts. Here, in our analysis we have combined $\left(\frac{\partial \Delta F^{S R K}}{\partial n_{i}}\right)_{V, T, n_{j}}$ and $-\ln \frac{Z}{Z_{0}}$ as "SRK $+\ln Z$ " contribution.

There is no association contribution for ions, but the association of solvent is affected by the presence of ions, so, the contribution from solvent association is also considered. $\Delta F^{A s s o}$ is due to the influence of the solvent. We name $\left(\frac{\partial \Delta F^{A s s o}}{\partial n_{i}}\right)_{V, T, n_{j}}$ as “Association" contribution.

$\Delta F^{D H}$ is the $\Delta F$ from ion-ion interaction, we name $\left(\frac{\partial \Delta F^{D H}}{\partial n_{i}}\right)_{\varepsilon_{r, V, T, n_{j}}}$ as " $D H$ " contribution. $\Delta F^{B o r n}$ is the $\Delta F$ from ion solvation, we name $\left(\frac{\partial \Delta F^{B o r n}}{\partial \varepsilon_{r}}\right)_{V, T, n_{j}}$ as "Born" contribution.

It needs to be pointed out that the relative static permittivity is composition-dependent. When obtaining the chemical potential of ions by differentiating the residual Helmholtz energy, the derivatives of relative static permittivity should be taken account into. We combined the $\left(\frac{\partial \Delta F^{D H}}{\partial \varepsilon_{r}}\right)_{V, T, n_{j}}\left(\frac{\partial \varepsilon_{r}}{\partial n_{i}}\right)_{V, T, n_{j}}$ and $\left(\frac{\partial \Delta F^{B o r n}}{\partial \varepsilon_{r}}\right)_{V, T, n_{j}}\left(\frac{\partial \varepsilon_{r}}{\partial n_{i}}\right)_{V, T, n_{j}}$ terms from both DH and Born as "Permittivity" contribution. 
In conclusion, the contributions of excess chemical potential for ion are divided into 5 parts, as summarized in Table 3.

Table 3. Contributions of activity coefficients in e-CPA.

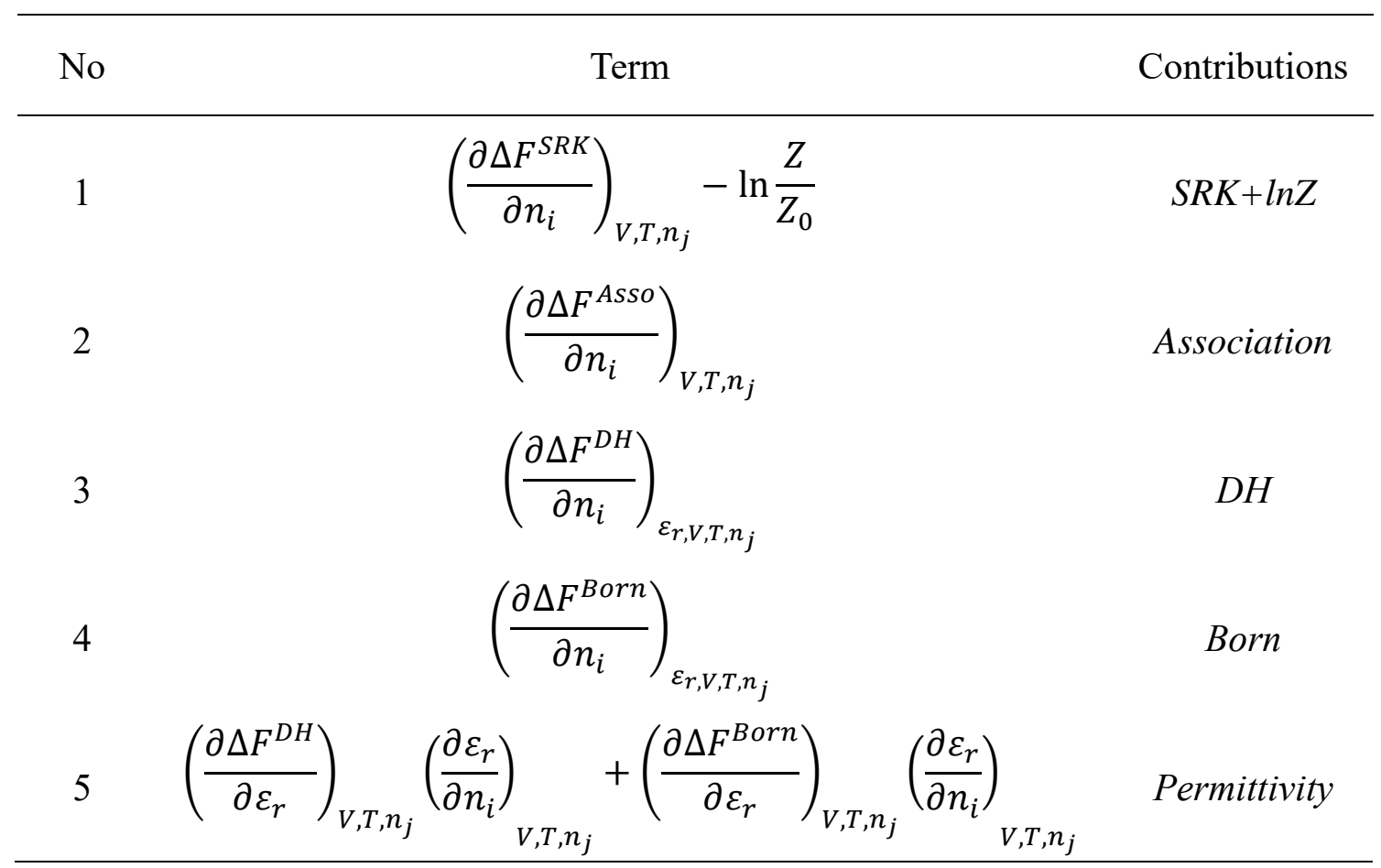

In this part, the ACI and mean ionic activity coefficients are calculated using e-CPA with the parameters from our previous work ${ }^{32}$. There are no e-CPA parameters for the $\mathrm{LaCl}_{3}+\mathrm{H}_{2} \mathrm{O}$ system, so this system is not considered. We present in Figures $10-17$ both the complete results for e-CPA and an analysis of the individual terms. 


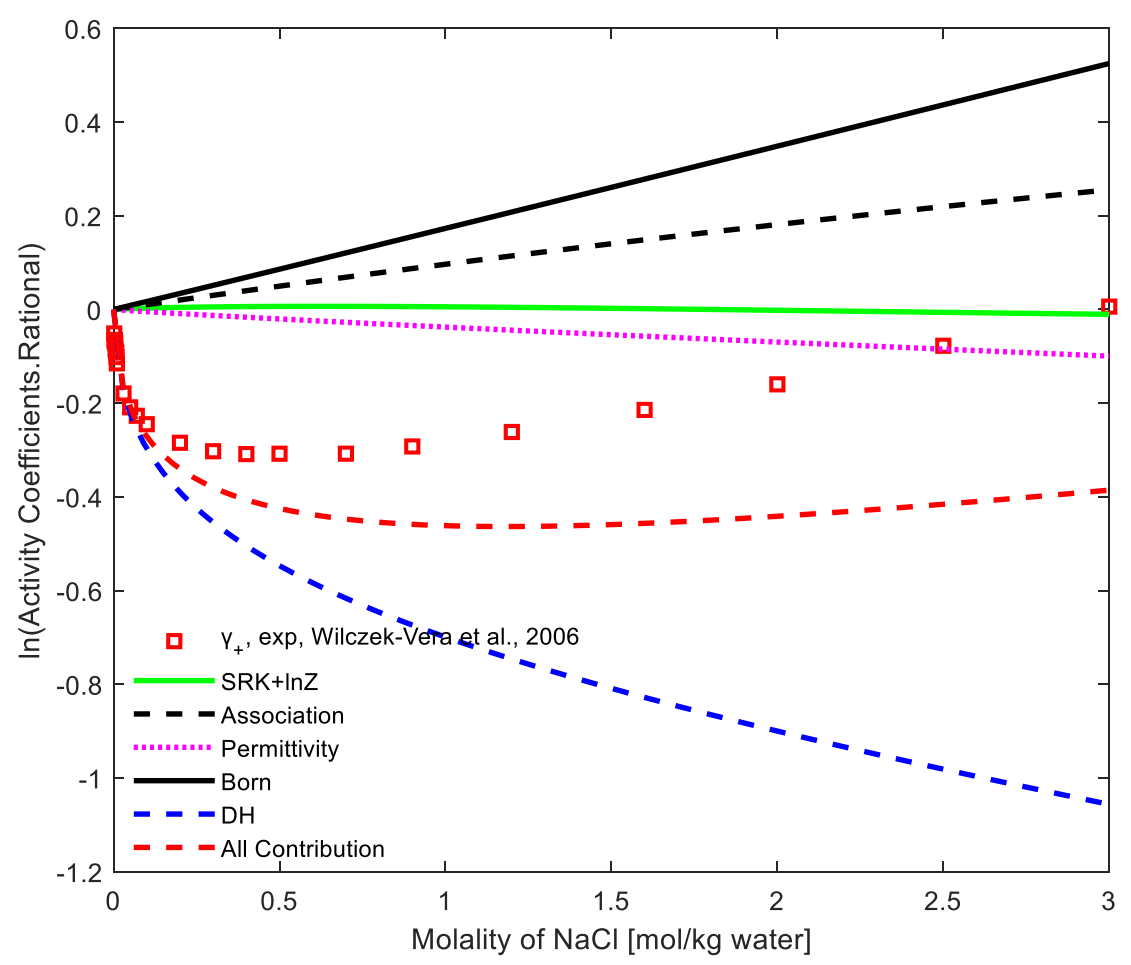

a. activity coefficients of cation ${ }^{10}$

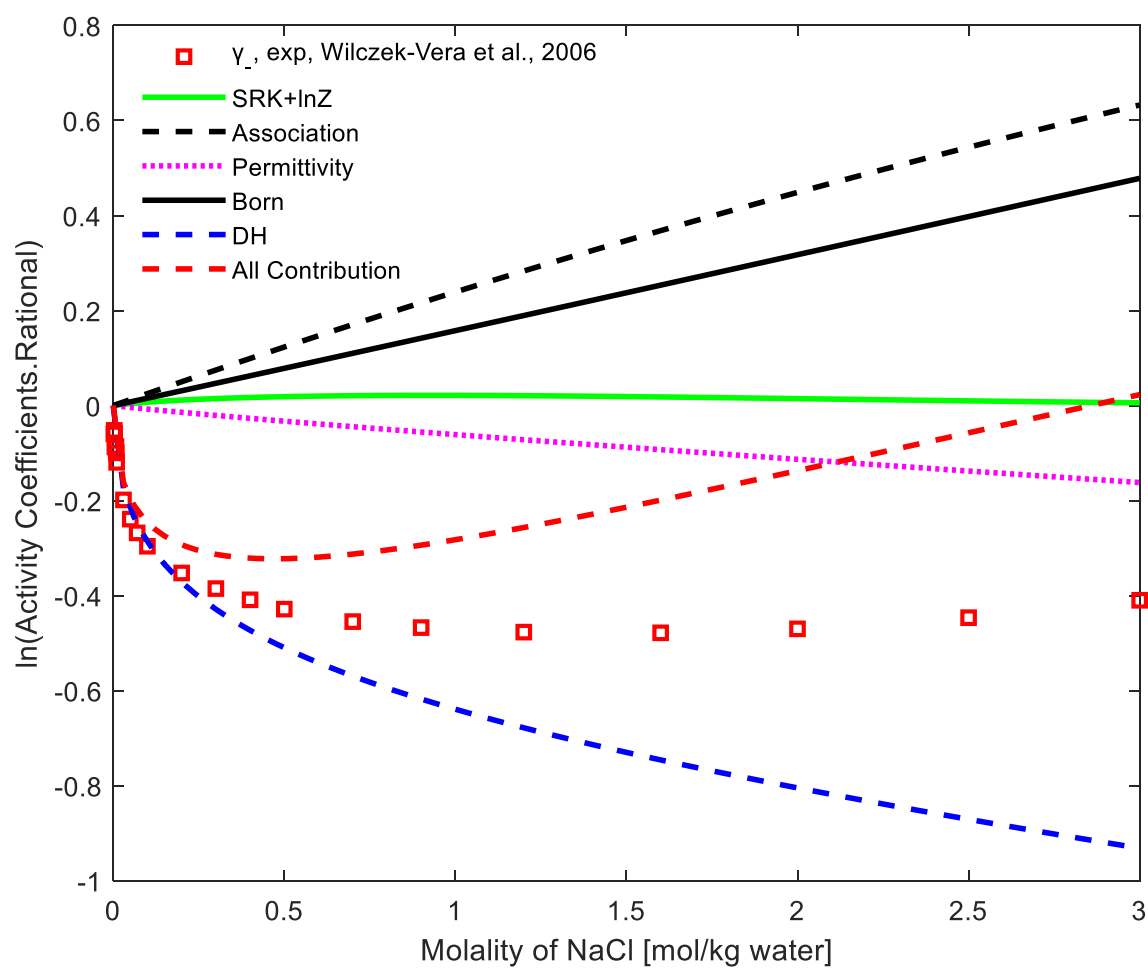

b. activity coefficients of anion ${ }^{10}$ 


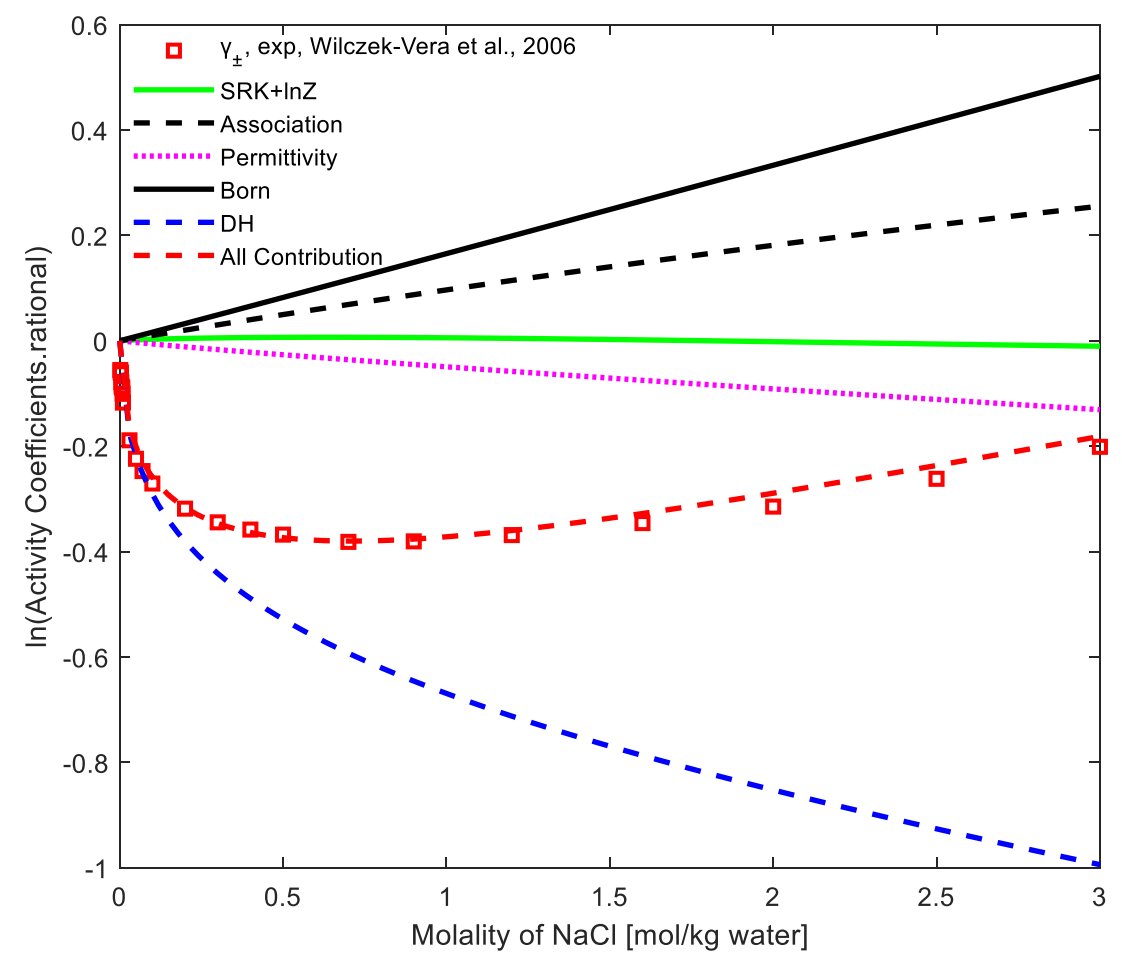

c. mean ionic activity coefficients ${ }^{10}$

Figure 10. e-CPA performance of ACI and mean ionic activity coefficients of $\mathrm{NaCl}$ in aqueous solution.

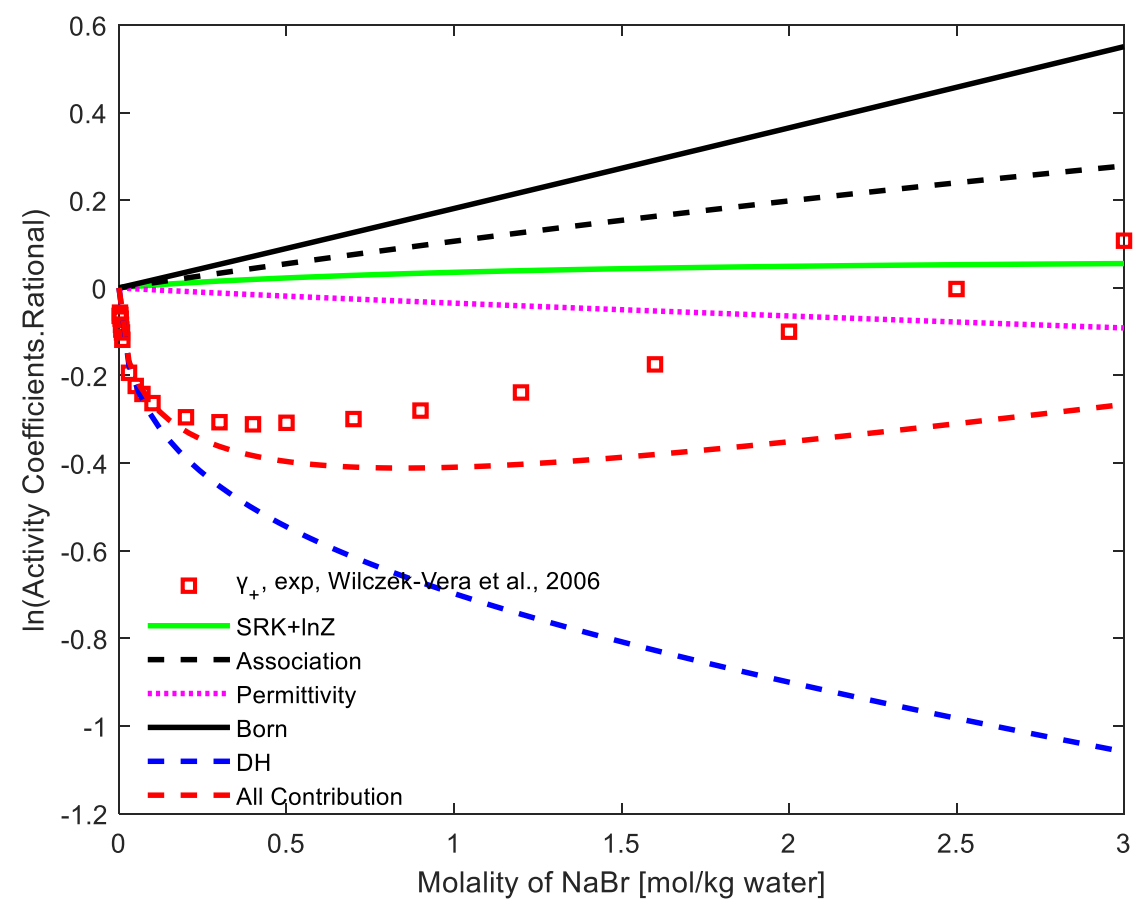

a. activity coefficients of cation ${ }^{10}$ 


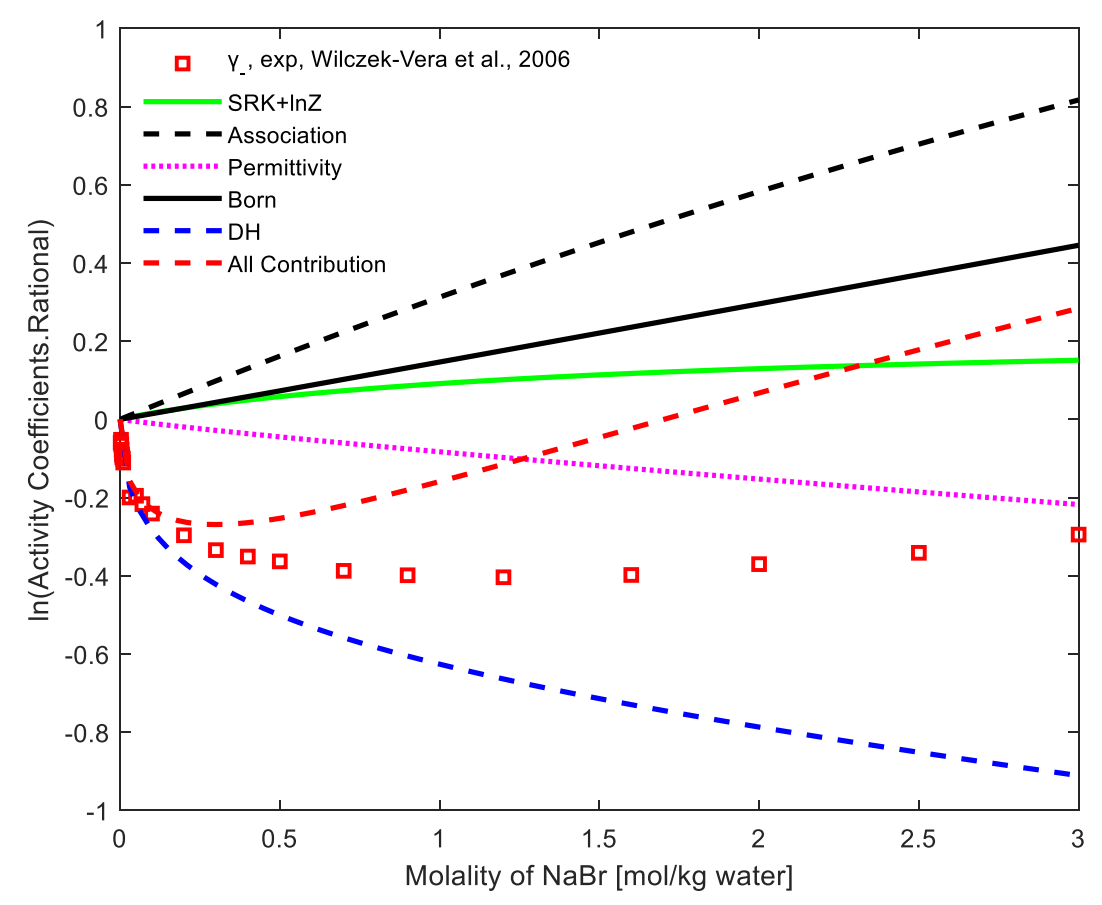

b. activity coefficients of anion ${ }^{10}$

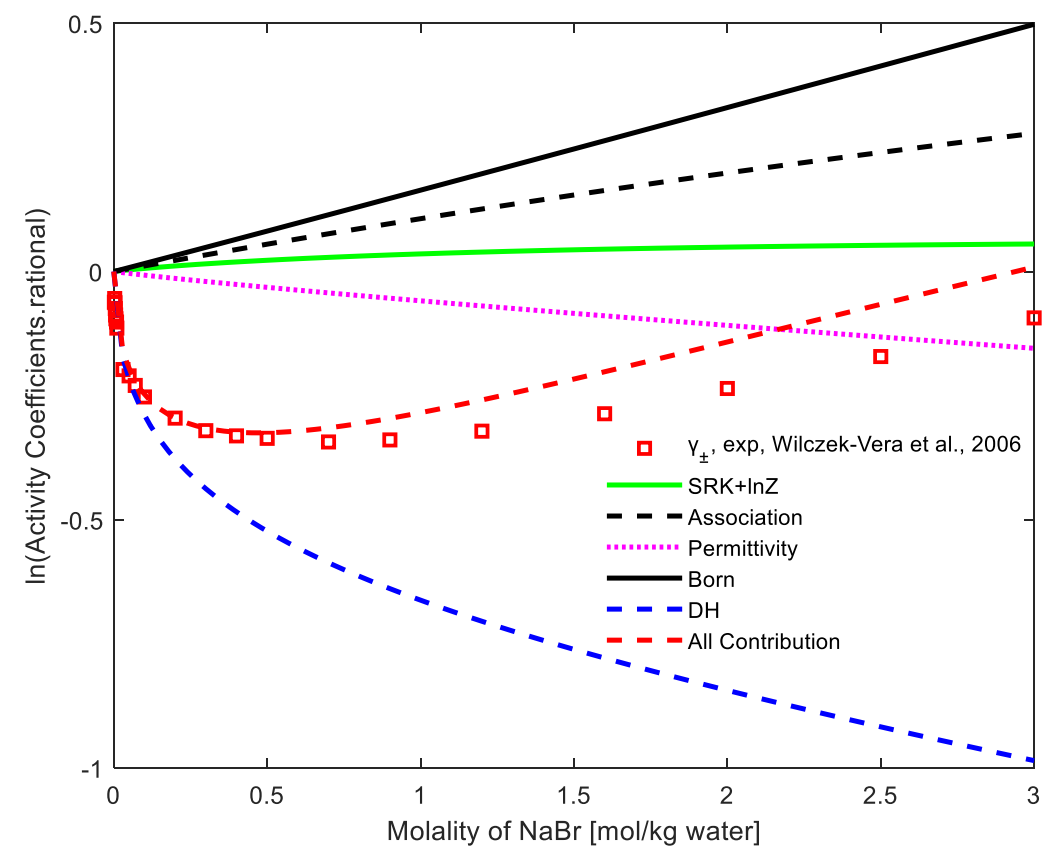

c. mean ionic activity coefficients ${ }^{10}$

Figure 11. e-CPA performance of ACI and mean ionic activity coefficients of $\mathrm{NaBr}$ in aqueous solution. 


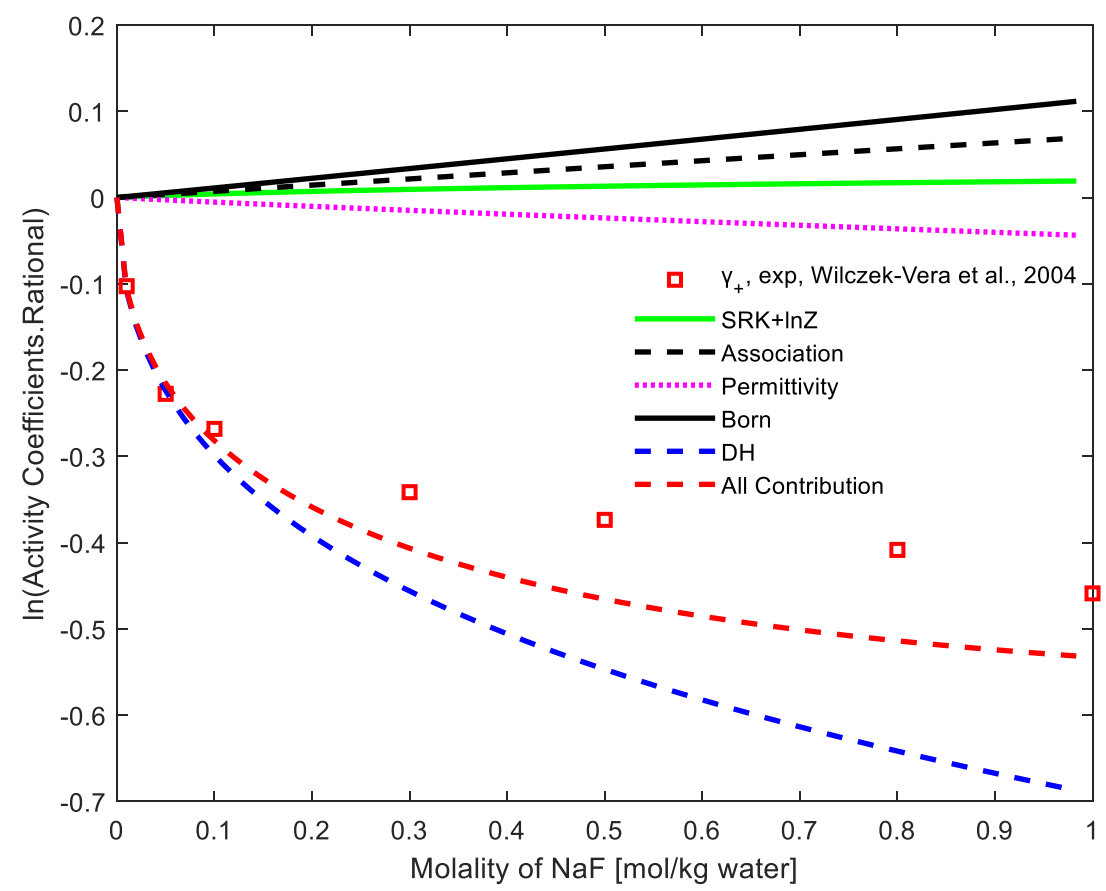

a. activity coefficients of cation ${ }^{9}$

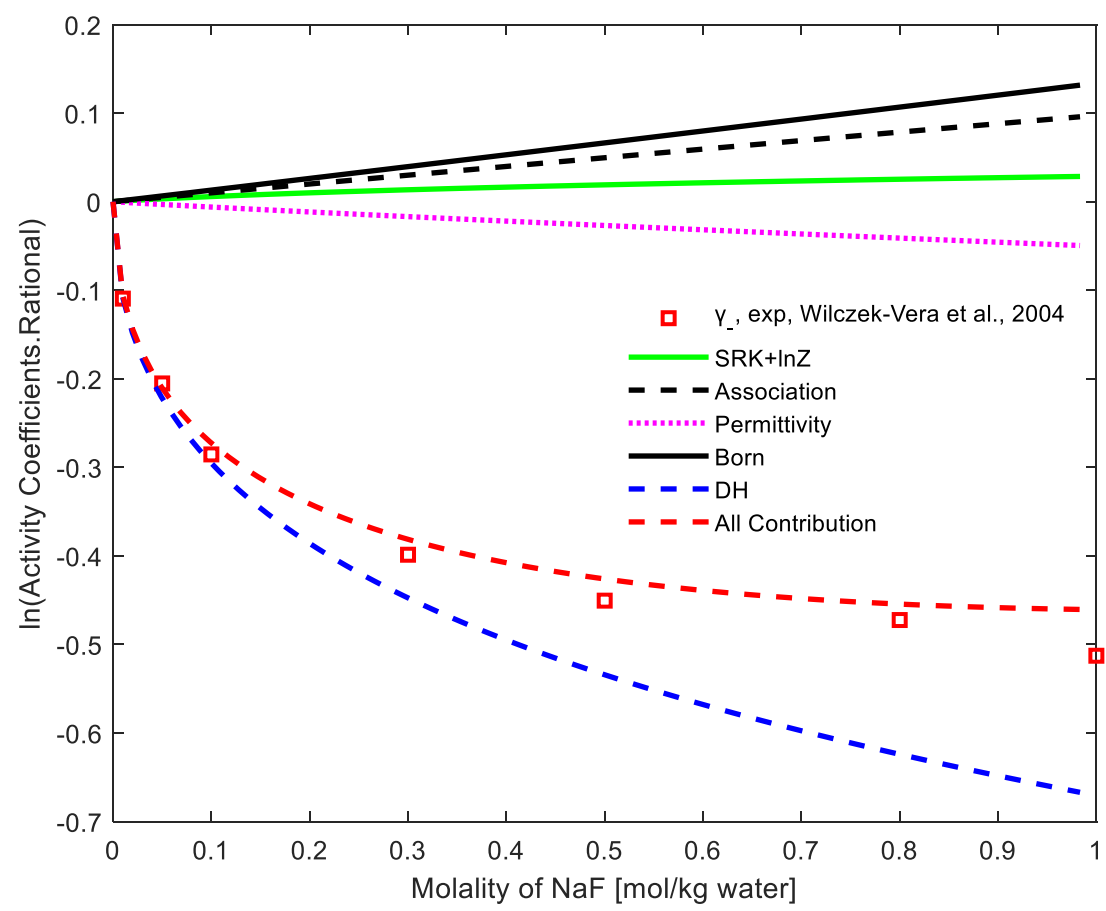

b. activity coefficients of anion ${ }^{9}$ 


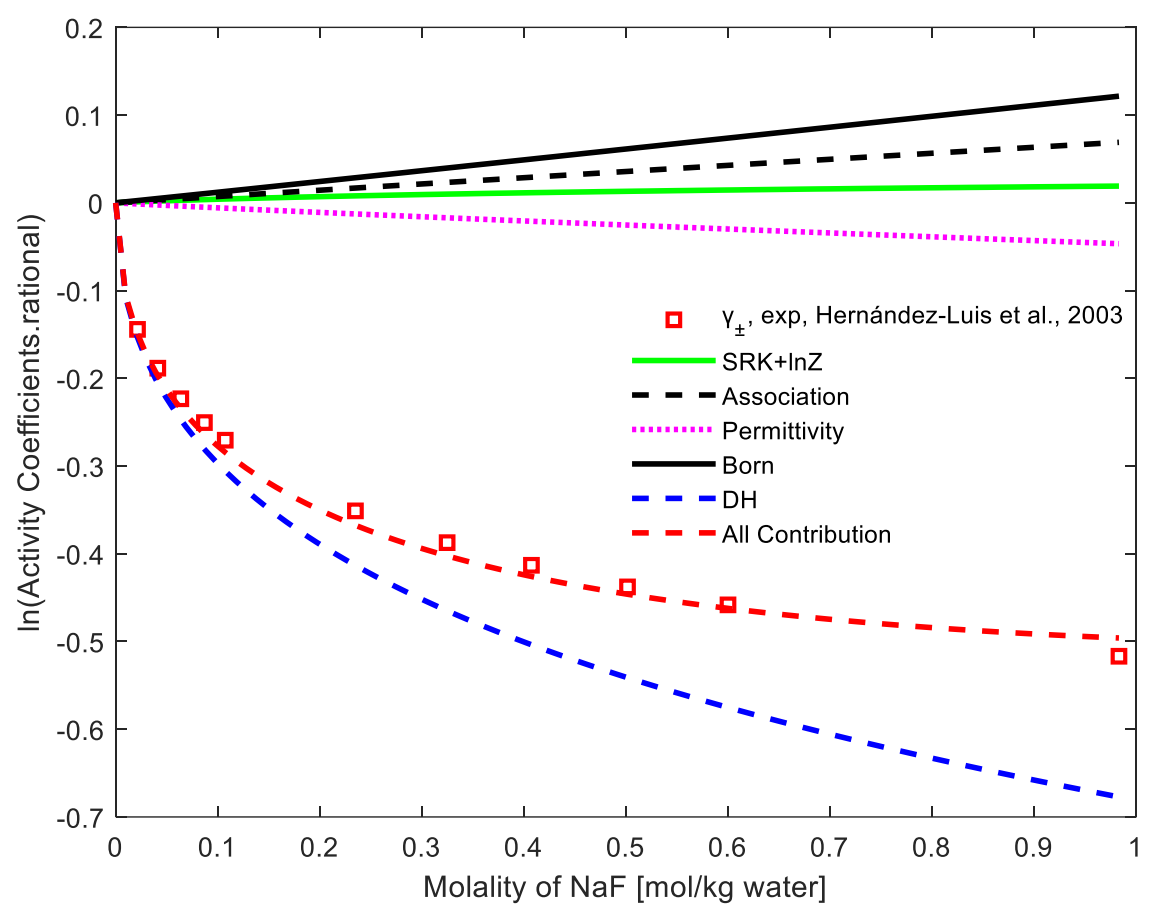

c. mean ionic activity coefficients ${ }^{53}$

Figure 12. e-CPA performance of ACI and mean ionic activity coefficients of $\mathrm{NaF}$ in aqueous solution.

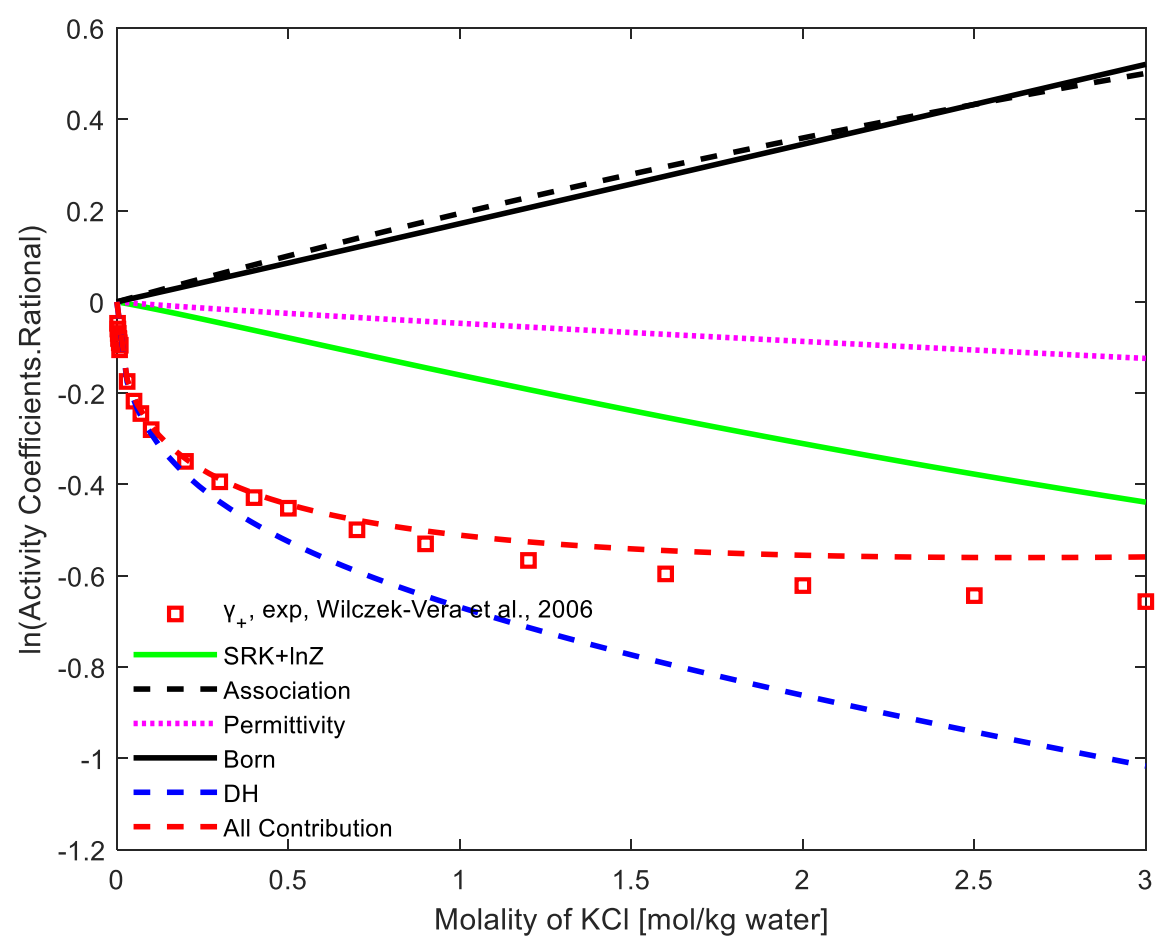

a. activity coefficients of cation ${ }^{10}$ 


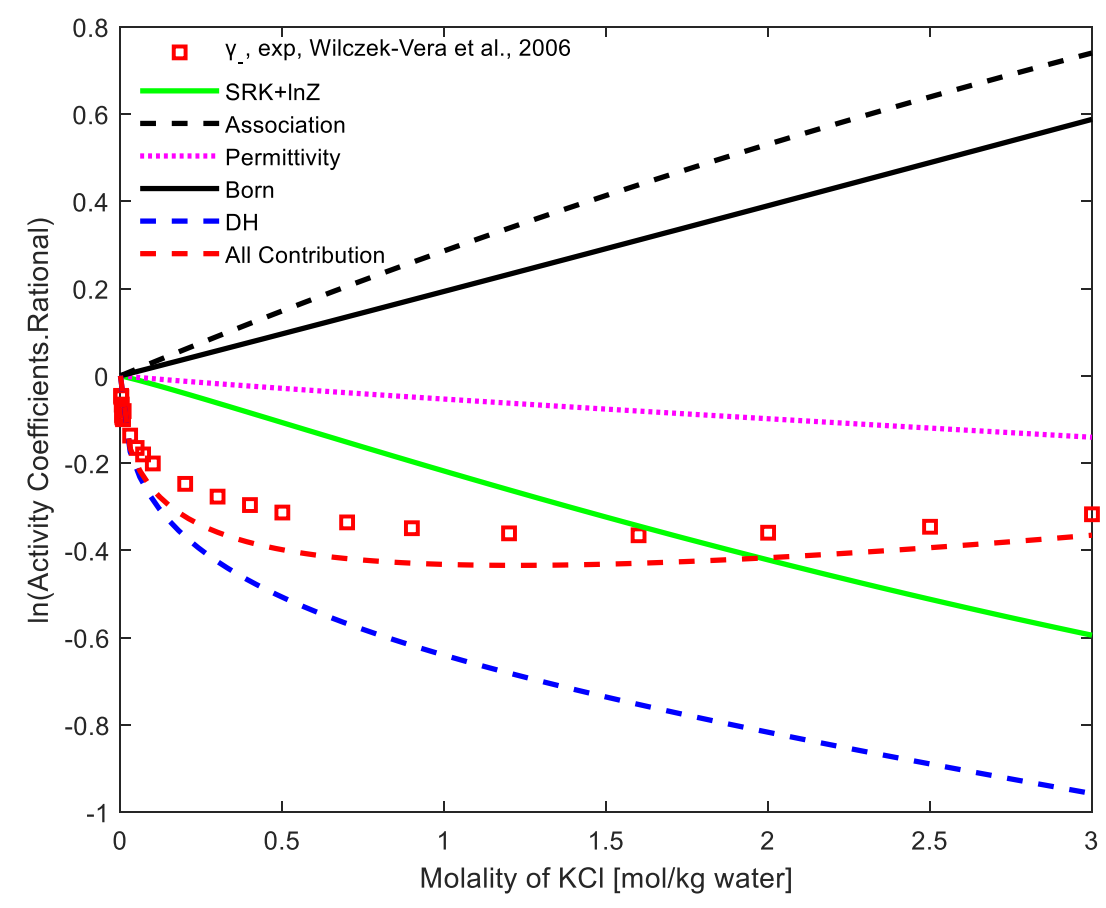

b. activity coefficients of anion ${ }^{10}$

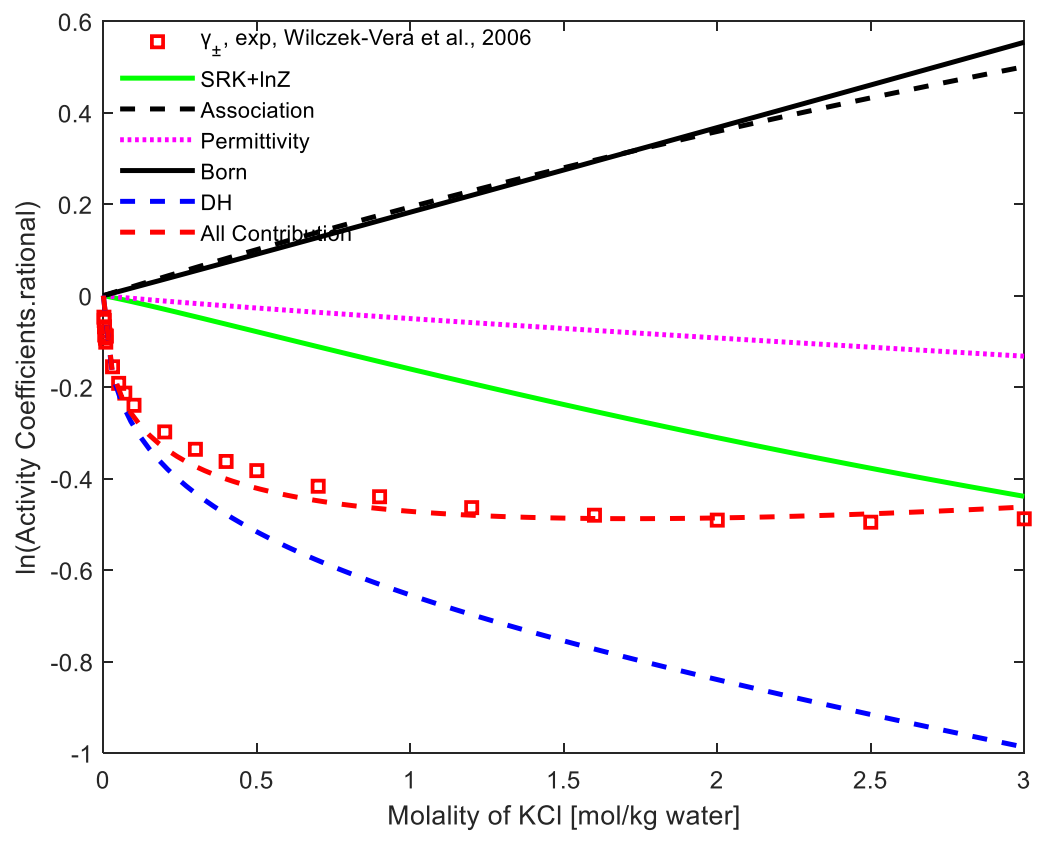

c. mean ionic activity coefficients ${ }^{10}$

Figure 13. e-CPA performance of $\mathrm{ACI}$ and mean ionic activity coefficients of $\mathrm{KCl}$ in aqueous solution. 


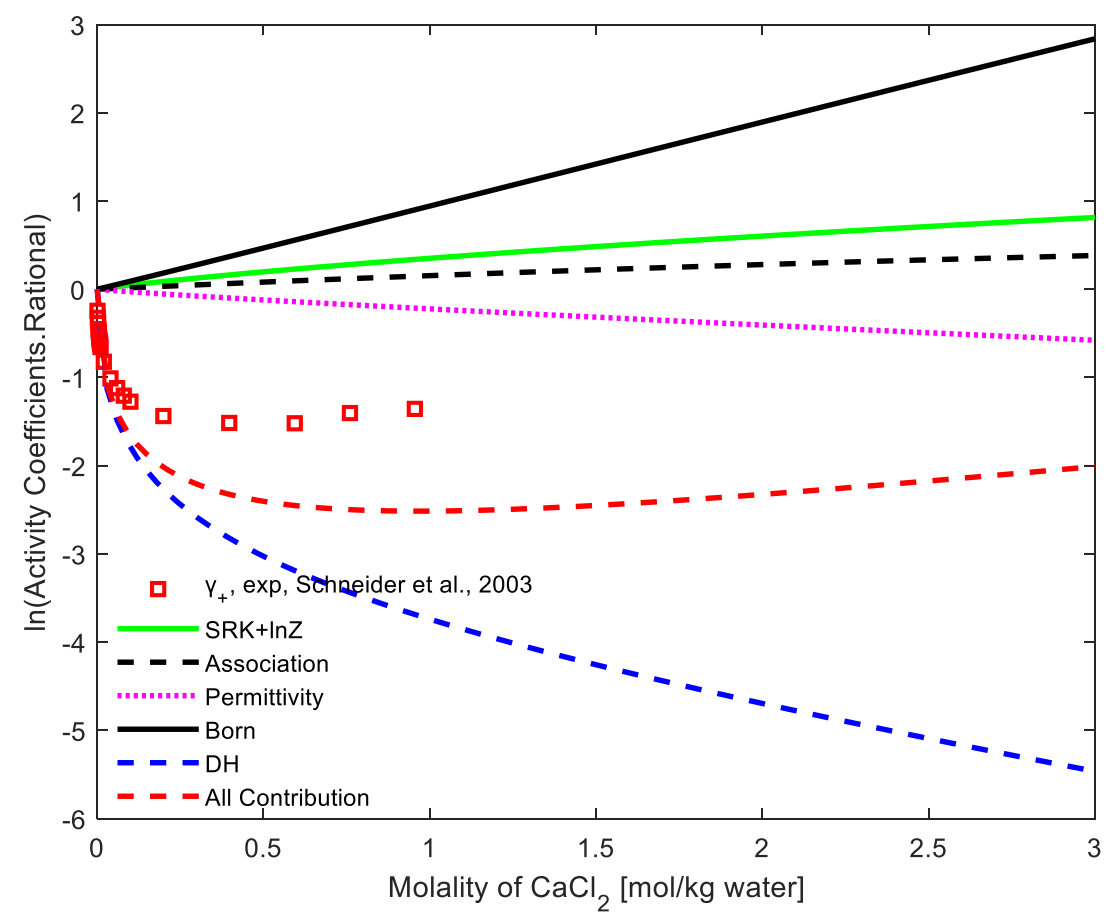

a. activity coefficients of cation ${ }^{54}$

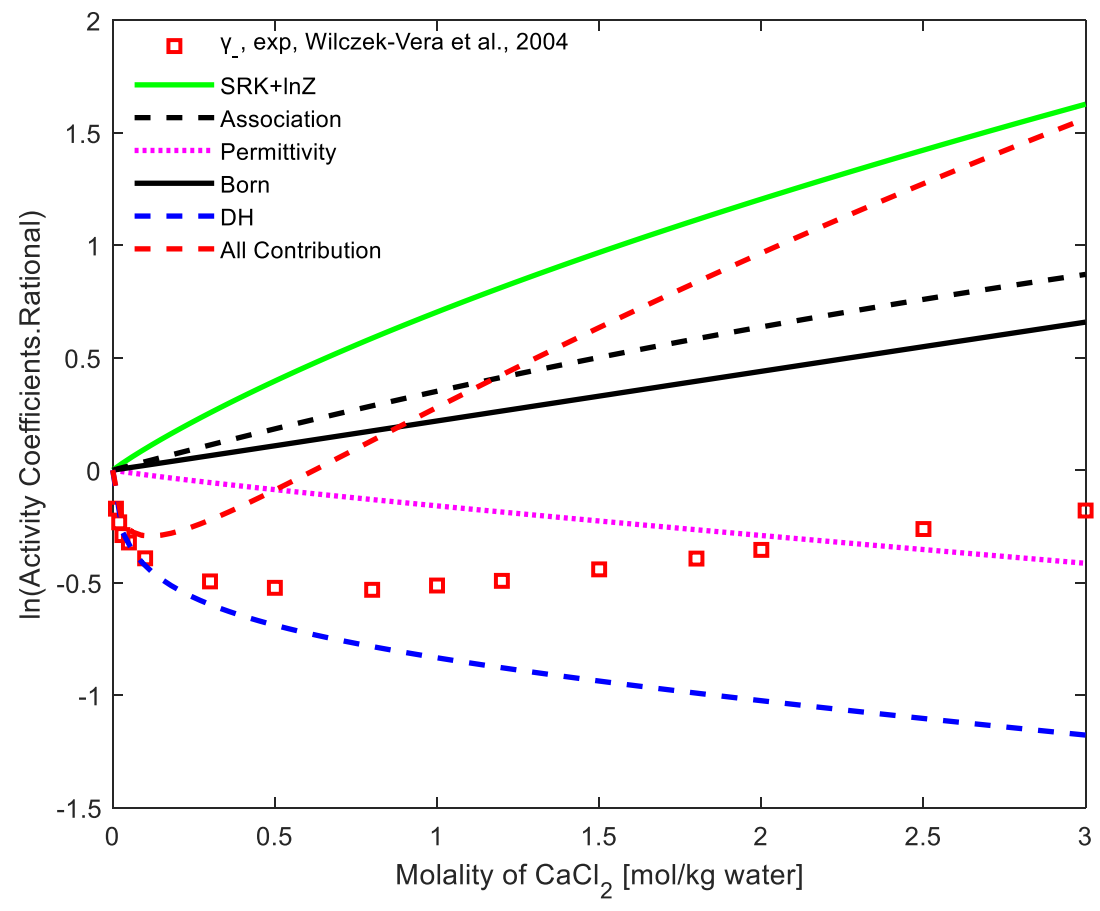

b. activity coefficients of anion ${ }^{9}$ 


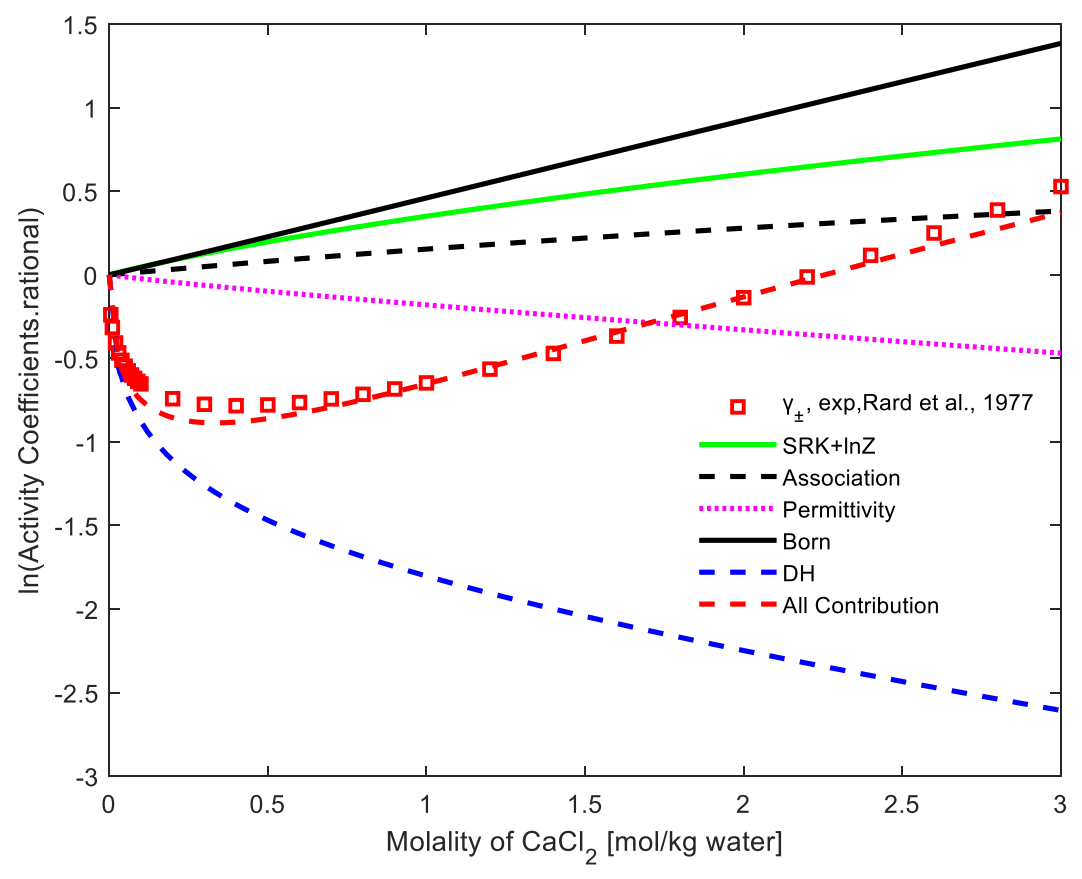

c. mean ionic activity coefficients ${ }^{55}$

Figure 14. e-CPA performance of $\mathrm{ACI}$ and mean ionic activity coefficients of $\mathrm{CaCl}_{2}$ in aqueous solution.

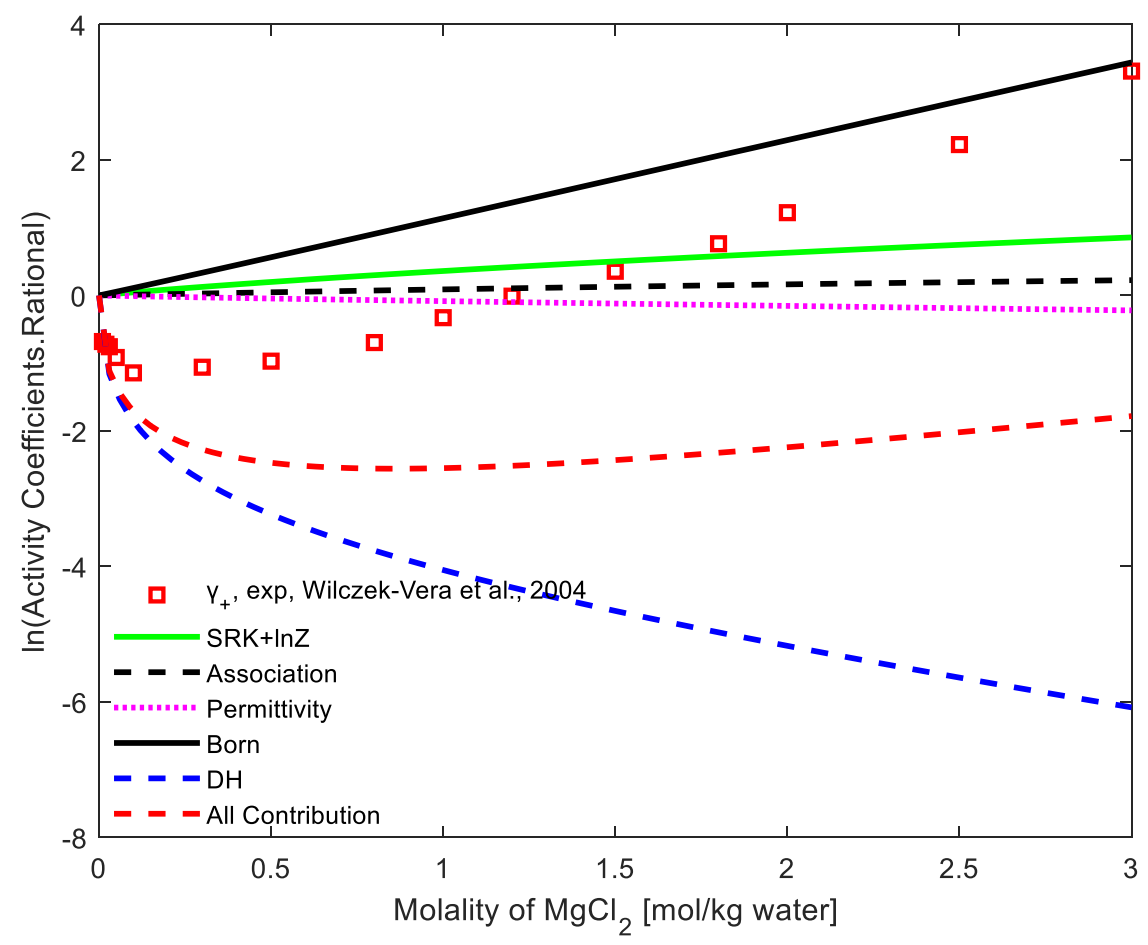

a. activity coefficients of cation ${ }^{9}$ 


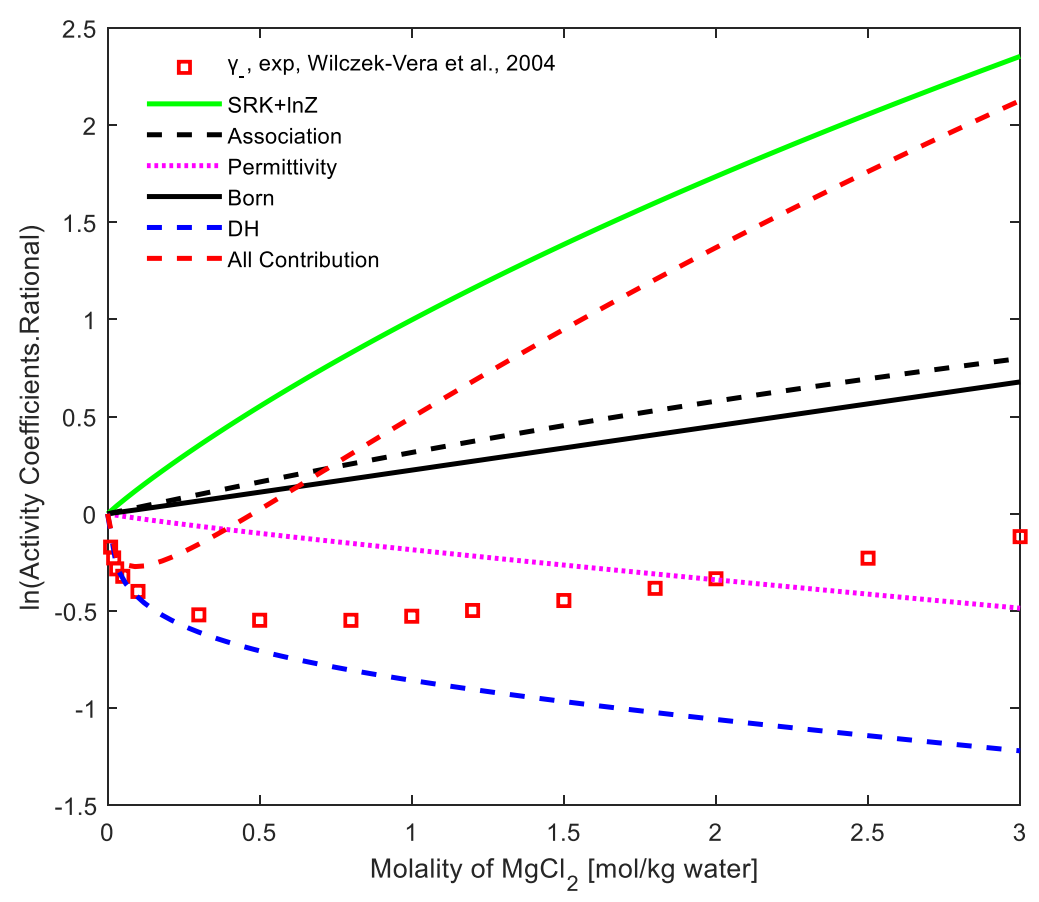

b. activity coefficients of anion ${ }^{9}$

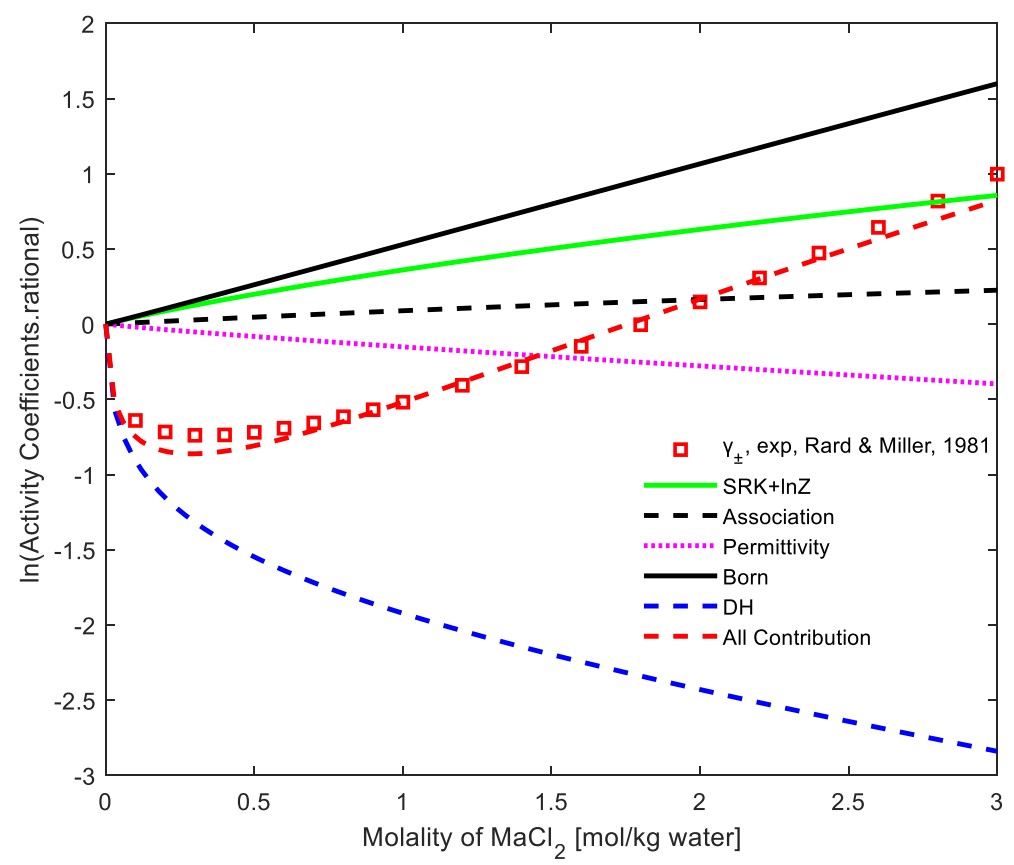

c. mean ionic activity coefficients ${ }^{56}$

Figure 15. e-CPA performance of ACI and mean ionic activity coefficients of $\mathrm{MgCl}_{2}$ in aqueous solution. 


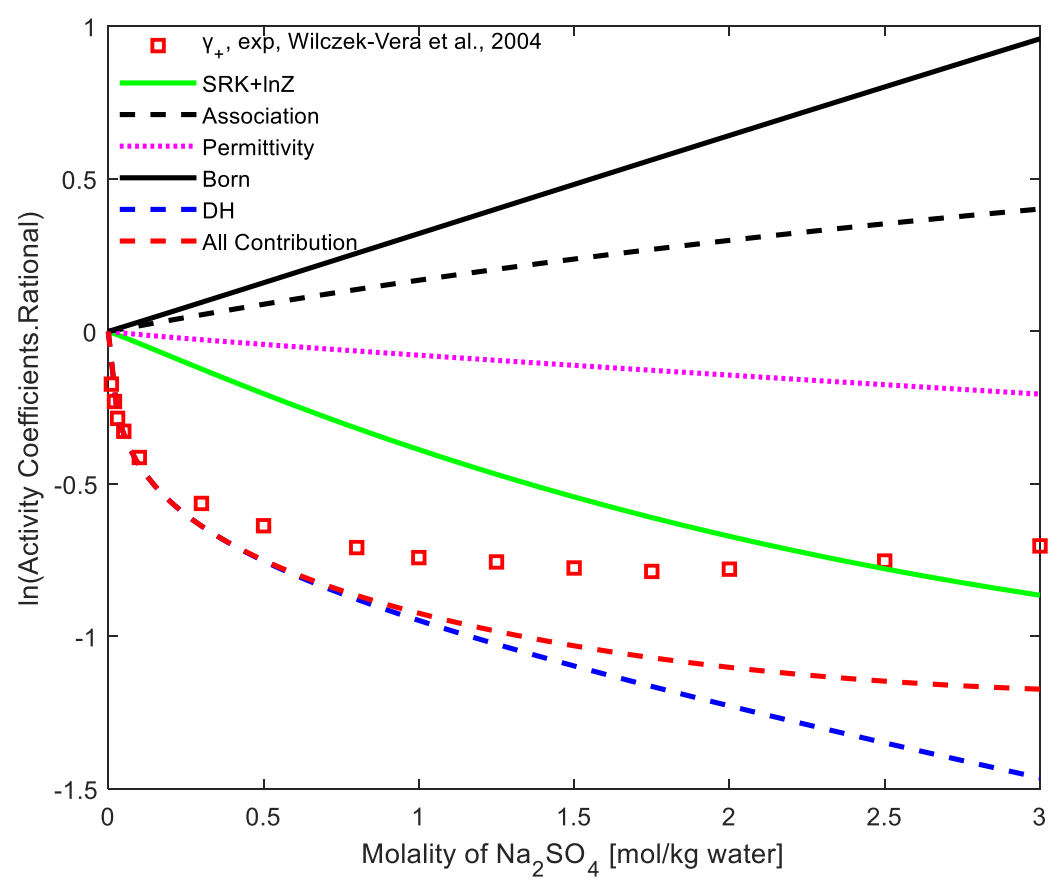

a. activity coefficients of cation ${ }^{9}$

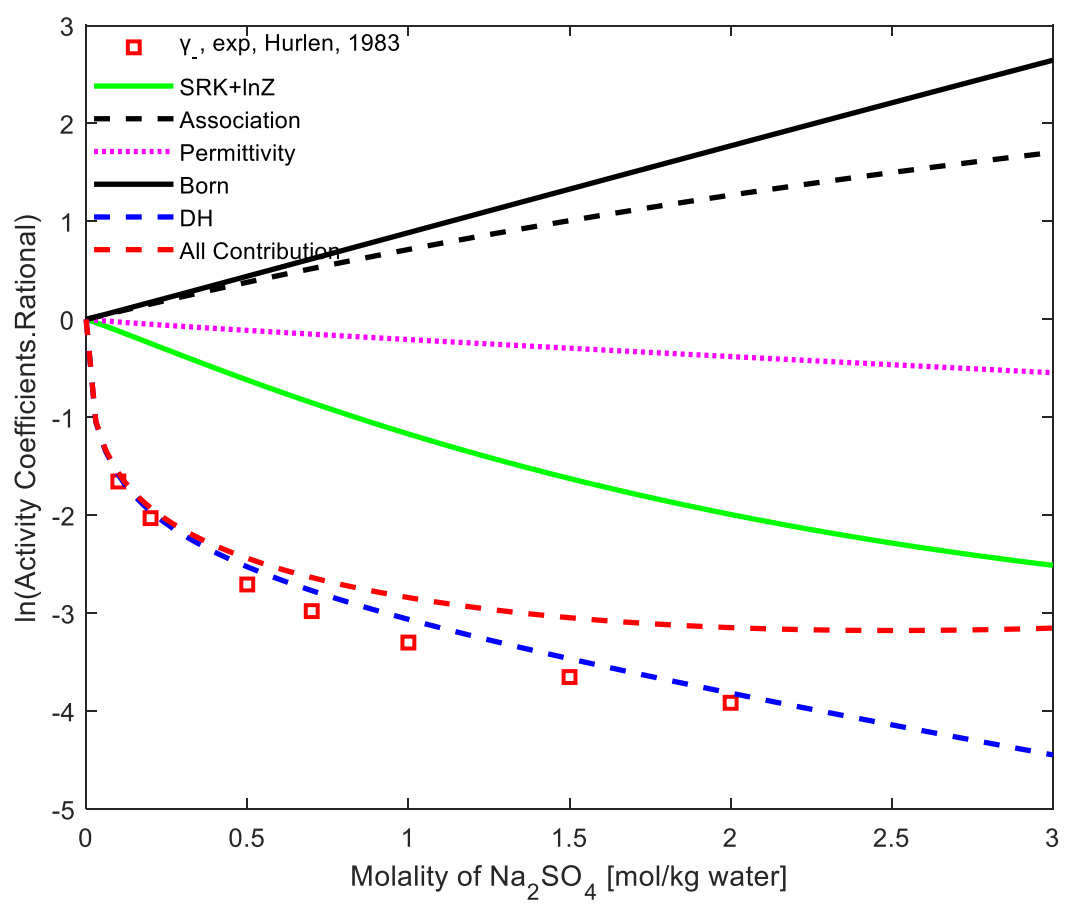

b. activity coefficients of anion ${ }^{57}$ 


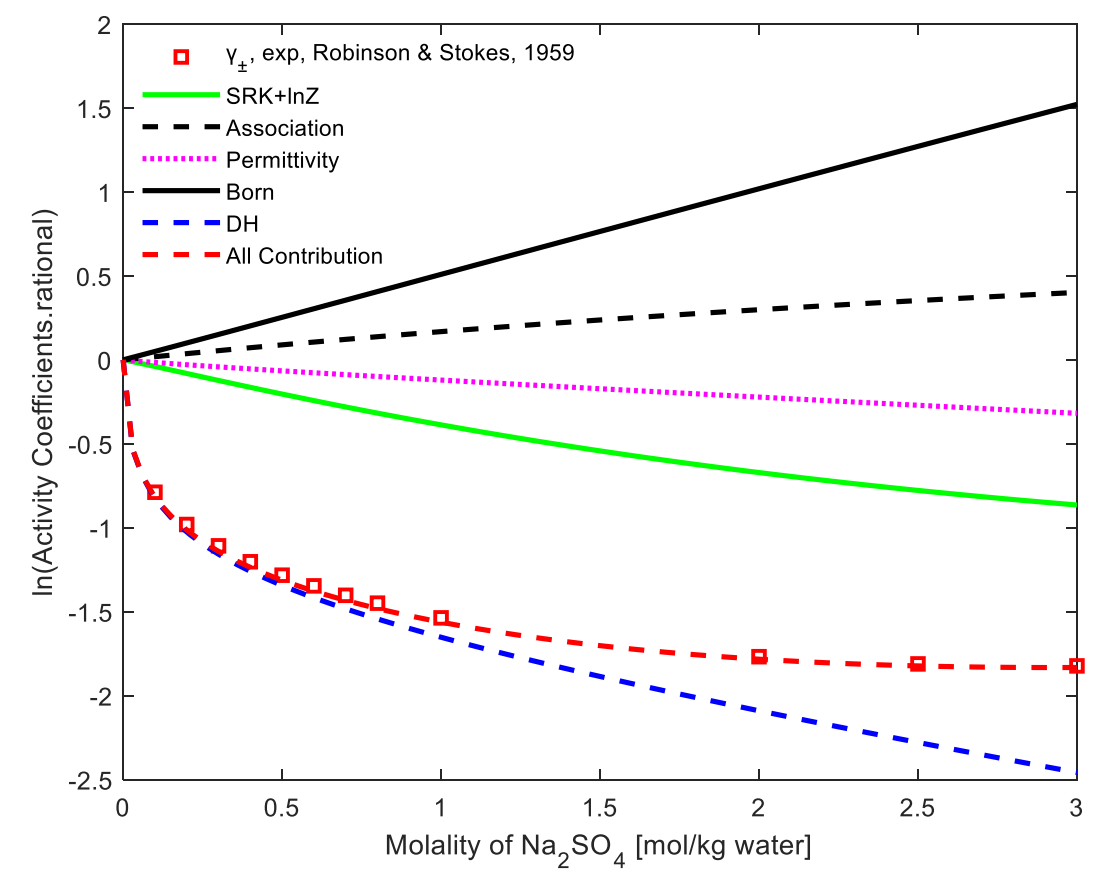

c. mean ionic activity coefficients ${ }^{36}$

Figure 16. e-CPA performance of ACI and mean ionic activity coefficients of $\mathrm{Na}_{2} \mathrm{SO}_{4}$ in aqueous solution.

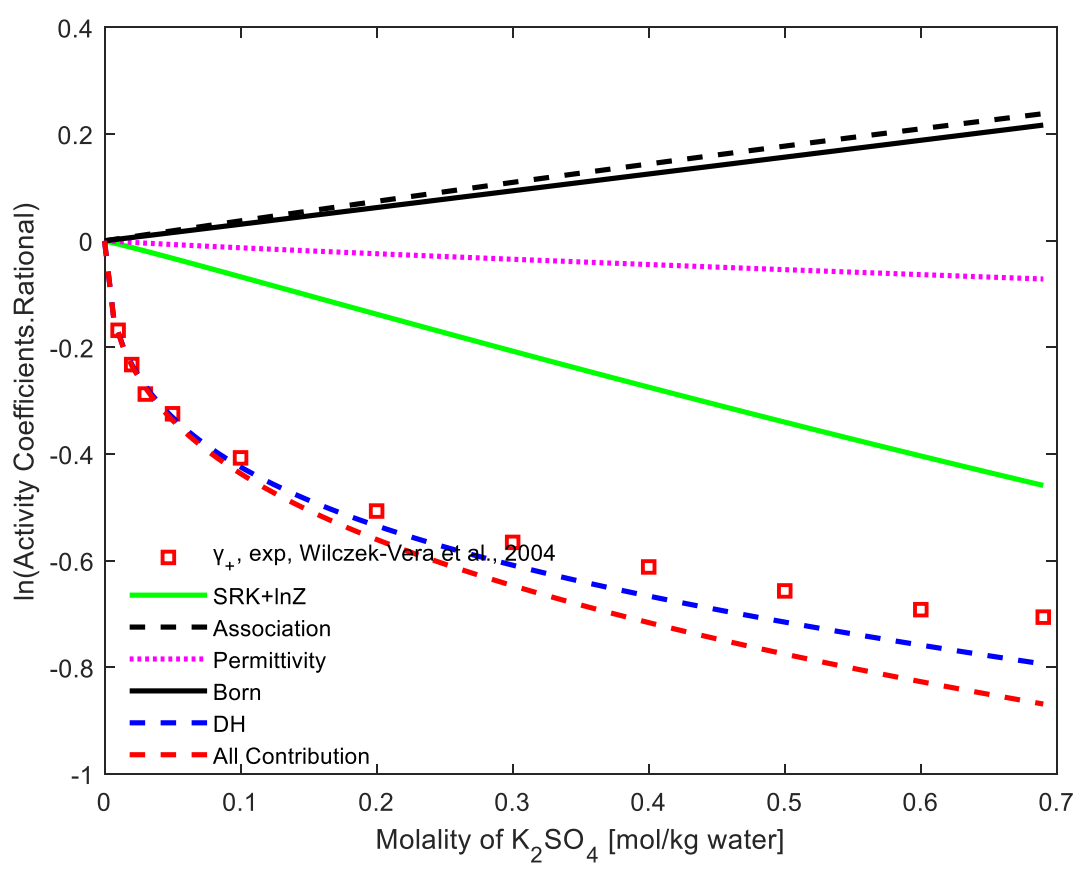

a. activity coefficients of cation ${ }^{9}$ 


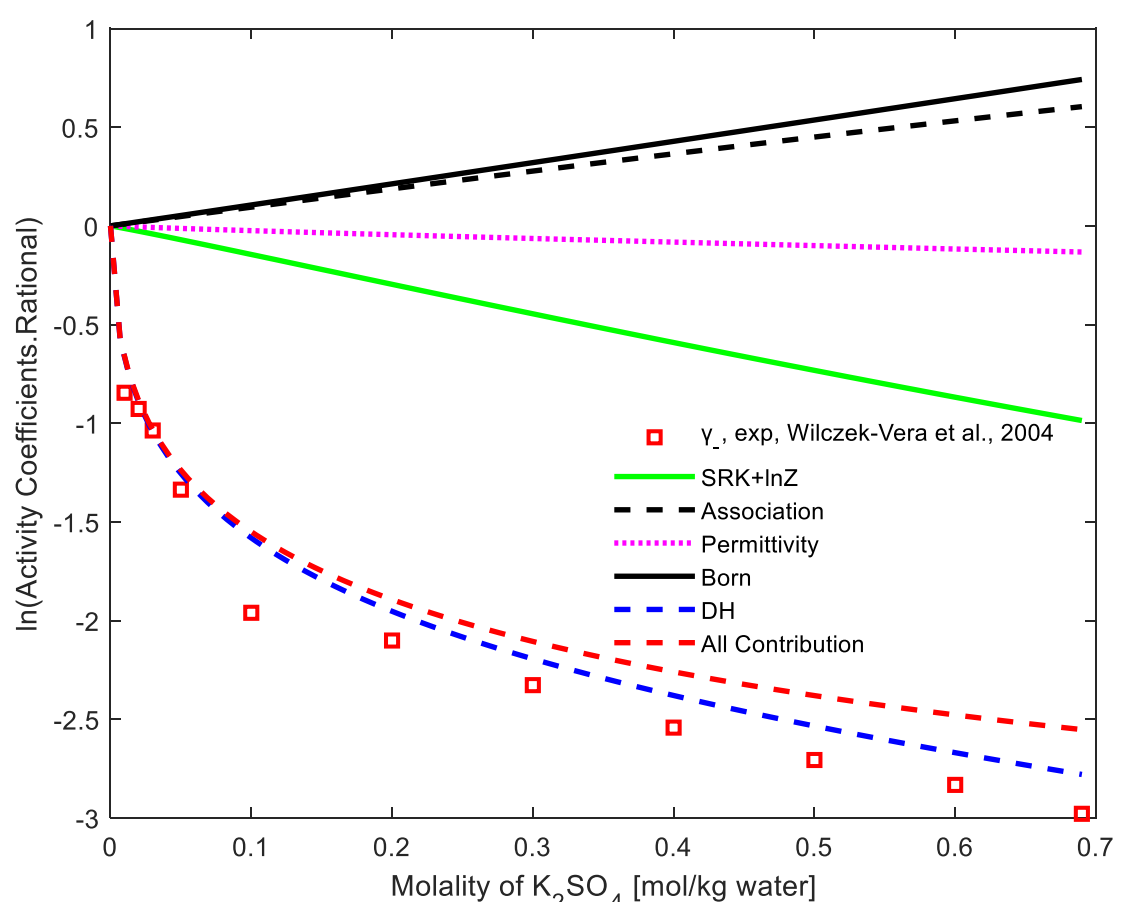

b. activity coefficients of anion ${ }^{9}$

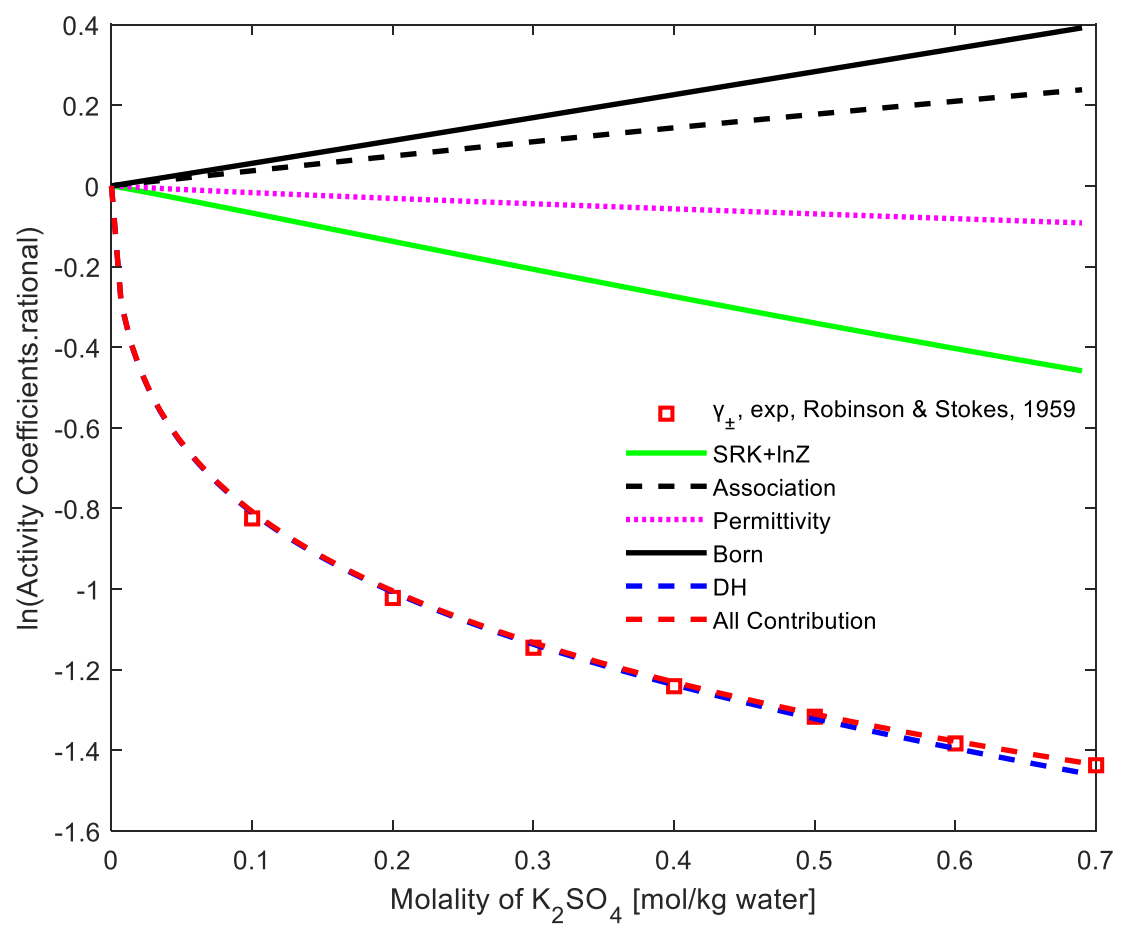

c. mean ionic activity coefficients ${ }^{36}$

Figure 17. e-CPA performance of ACI and mean ionic activity coefficients of $\mathrm{K}_{2} \mathrm{SO}_{4}$ in aqueous solution. 
Because the e-CPA adjustable parameters are obtained by fitting the mean ionic activity coefficients, the e-CPA model can give good agreement for the mean ionic activity coefficients. From Figures 10-17, it can be seen that, the present version of e-CPA with the parameters optimized from mean ionic activity coefficients cannot provide, in general terms, very accurate values for the ACI.

What we observe is that there is an error for both the activity coefficients of cation (systematic underestimation) and the activity coefficient of anion (systematic overestimation). The two effects cancel out when the activity coefficients of cation and anion are combined to yield the mean ionic activity coefficients, but the problem remains for the ACI. The DH and Born effects are still large and opposing, as we have seen in the previous section, but the values are different compared to those from the II+IW theory. For most cases, the "SRK $+\ln Z$ " term is important, and affects the results significantly. Though the contributions from relative static permittivity are small, this contribution cannot be ignored. Finally, the association contribution is also of importance, especially at higher molalities (also the case for the SRK term). The physical (SRK) and association terms have often (but not always) opposing trends (SRK negative and association positive), again especially pronounced at higher concentrations.

Still the results are not identical for all salts. While we see significant problems, as discussed above, for $\mathrm{NaCl}$ and $\mathrm{NaBr}$ as well as for $\mathrm{CaCl}_{2}, \mathrm{Na}_{2} \mathrm{SO}_{4}$ and $\mathrm{MgCl}_{2}$, the results for $\mathrm{NaF}$ and $\mathrm{KCl}$ are overall fine (and perfect for the anion coefficients). Even 
for $\mathrm{K}_{2} \mathrm{SO}_{4}$ (despite seeing again the under/over estimation of, respectively, the activity coefficients of cation and anion), the overall performance of e-CPA is satisfactory for both the single and mean ionic activity coefficients. Good agreement with activity coefficients for certain individual ions may be accidental, but this illustrates that e-CPA can perform qualitatively well for both mean ionic activity coefficients and ACI.

The electrostatic interaction terms of e-CPA are $\mathrm{DH}$ and Born, and these are used in the calculations shown in section 5.1. The solvent relative static permittivity of electrolyte solutions is critical for calculating the electrostatic interactions. In the original e-CPA, the relative static permittivity is calculated from a new model which eliminates the kinetic depolarization (the values are larger than the experimental values). In the II+IW theory calculations, the relative static permittivity is calculated from the correlations of experimental data. The calculation of liquid density is also included in the entire e-CPA framework. That means that the II+IW theory and the e-CPA EoS use different values of relative static permittivity and liquid density, so, the II contribution and IW contributions are somewhat different in the II+IW theory and the e-CPA EoS. Apart from the ion-ion and ion-water interactions, the e-CPA EoS also considers physical contributions (e.g. SRK-term, association), and the adjustable parameters (using in this section (5.2)) are obtained by fitting to mean ionic activity coefficients. II and IW contributions alone cannot reflect all interactions in electrolyte solutions, and by re-parameterizing also including ACI data, e-CPA may potentially improve the performance for electrolyte solutions. 


\section{Discussion}

\subsection{Activity Coefficients of Individual Ions}

Some further reflections on the various models' characteristics and possible connection to their performance follow in this section.

The DH-SiS model ${ }^{18}$ neglects the non-electrostatic effects, such as the core potential. Fraenkel ${ }^{18}$ stated that only the size of the ion and the closest distance of the ion to its counterion are necessary individual factors for evaluating the ACI. The DH-SiS model is an extension of the DH theory, and it considers the ion and the counterion as having two shells of different diameters. Compared with the II+IW model, in the DH-SiS calculations, the "closest distance of the ion to its counter-ion" is used instead of the crystallographic ion size, and the pure water permittivity is used instead of a concentration-dependent permittivity. Fraenkel ${ }^{18}$ also stated that there is no need to consider ion solvation, ion pairing, and core effects in computing single-ion activities. For each activity-concentration curve, the $\mathrm{PF}$ model ${ }^{13}$ requires three unchanged parameters, which are associated with the Born radius.

In the II+IW model, the ACI are a result from the competing effects of the II and IW interactions. Pauling radii and Born radii are used in II and IW calculation respectively, and relative static permittivity (concentration-dependent) are used in calculations. The II+IW model does not contain any adjustable parameter, and as all the parameters used in the model are taken from experiments, the results can be considered to be entirely predictive. 
$\mathrm{DH}$ treats ions as point charges with a minimum separation distance. In the original DH theory, the distance of closest approach between ions is used, which is expected to be larger than the radius of the ion. MSA treats all ions as charged hard spheres, while the MSA model includes the effect of an excluded volume on the electrostatic interactions. The EDH theory incorporates the concentration dependence of electrolyte solution static permittivity. In our II+IW calculations, a concentration-dependent permittivity is used in the DH and MSA calculations in order to extend the concentration range where the models are applied, and the crystallographic ion size (Pauling radii) is used. In the EDH and MSA models, the same ion radius is used for activity coefficients of cation and anion. In the DH term of e-CPA, the cation radius and anion radius are used for activity coefficients of cation and anion respectively. But this difference is not reflected in the results.

The II+IW theory has a solid physical background, but it has limitations, e.g. the II+IW approaches do not always give accurate prediction of ACI for highly concentrated solutions. This may be partially explained by the fact that physical interactions are also important at high concentrations.

Compared with the II+IW theory, CPA (SRK+Association) contribution is the extra contribution in e-CPA. The performance for ACI is different for the II+IW theory compared to the original e-CPA. One of the main factors is the values of relative static permittivity. The importance of kinetic depolarization is always a debatable topic, and the elimination of kinetic depolarization needs more discussion. Another reason for the differences is the inclusion in e-CPA of the "physical" terms from SRK and the 
association contribution.

Finally, the Born equation provides a simple analytical formula for molecular electrostatic energy in the presence of implicit solvent ${ }^{59}$. The accuracy depends a lot on the values of the so-called effective Born radii. The usual assumption that a given ion has the same size in any compound is partially true ${ }^{51}$.

\subsection{Analysis of Terms of Models}

A few literature studies with complete e-EoS also provide an investigation of the relative size of the terms (often as contributions to ion or mean ionic activity coefficients, sometimes also as contributions to water activity and Z). These analyses for complete e-EoS are compared to the results from the II+IW theories (either from literature or from this work).

The first relevant study we have identified is from Simon et al. ${ }^{60}$ (Prausnitz group) for their e-EoS which includes SRK for physical interactions and both DH and Born terms. The relative static permittivity used is function of both temperature, solvent and ion composition. A single plot is shown with an analysis of terms, which is not discussed in the manuscript. The DH has a large negative contribution to the mean ionic activity coefficient and the Born the opposite (large and positive). Both terms start from zero and become more important at higher concentrations. The SRK contribution is smaller, appearing to peak at intermediate concentrations.

As for the group of de Hemptinne, such analysis we have found both in the 2008 paper $^{61}$ (Inchekel et al.) using e-CPA and 10 years later in the 2018 paper (Ahmed et 
al. ${ }^{62}$ with the e-PPC-SAFT EoS. The two models use slightly different physical terms, but both use the MSA equation for ion-ion interactions and the Born term for the ion-water effects. In both cases, a composition/temperature-dependent relative static permittivity is used. For water- $\mathrm{NaCl}$ and with both models, the Born term has a large positive increasing with concentration value, while the MSA has a large negative decreasing with concentration value. The two terms appear, thus, to be of almost equal significance when a composition-dependent relative static permittivity is used. If the relative static permittivity was independent of ionic concentration, then the Born term's contribution to the activity coefficient would be zero, forcing the other terms to counter-balance the ion-ion MSA term, especially in order to obtain the positive slope of the mean ionic activity coefficient at higher concentrations. The other terms are actually smaller, with the association term being of some significance esp. at higher concentrations. In the 2018 paper, the authors also present results for $\mathrm{NaF}$. The picture is different with the Born term being much smaller and the MSA and association \& chain terms appearing to be of same significance (and opposite trends).

In another recent study, Shahriari and Dehghani ${ }^{63}$ made an analysis of their e-EoS terms; an electrolyte PC-SAFT model with the DH theory and a Born term and a relative static permittivity function of composition according to the theory proposed by Maribo-Mogensen et al. Their model contains, unlike the previously mentioned models, also ion-ion association and it includes three adjustable parameters fitted to mean ionic activity coefficient data and apparent molar volumes. They present an analysis of terms for the compressibility factor, Z, as well as for the Helmholtz free 
energy temperature derivative of $\mathrm{NaCl}$ and the contribution to $\mathrm{Na}+$ chemical potential temperature derivative (versus temperature in the last two cases at infinite dilution). In the Z-molality plot, the DH contribution has an increasing large positive value and the Born a decreasing large negative value, thus both terms are equally significant as shown before, simply now with opposite signs compared to the activity coefficient plots. For the two T-derivatives mentioned, it is actually in both cases the Born term that dominates and it changes a lot with temperature. The other terms have smaller values and, moreover, they change less with temperature; with the association term being of some importance.

A similar conclusion is reached in the work of Selam et al. ${ }^{64}$ with their electrolyte SAFT-VR Mie EoS. This model includes (in addition to SAFT-VR physical/association terms), the full $\mathrm{DH}$ term for electrostatic effects and the Born term with composition-independent relative static permittivity. Two ion-specific parameters (dispersion energy and diameter) are fitted to mean ionic activity coefficients and densities, but for more accurate results at high temperature, it is needed to use a temperature-dependent ion-water dispersion interaction parameter. The authors show that the DH contribution to the chemical potential is negative, slightly higher at higher temperature. If temperature-independent interaction parameters are used then the non-electrostatic term is of same sign as DH and increasing with temperature (which is surprising), but with temperature-dependent dispersion parameter, the sign of the term is positive and decreasing with increasing temperature. 
Finally, the great importance of Born term (on the activity coefficients this time against $\mathrm{P}$ ) for $\mathrm{NaCl}$-water is shown for another EoS proposed recently by Zuniga-Hinojosa et al. ${ }^{65}$ The MSA term is smaller, while the physical term (a cubic term) has also some contribution.

The above are some of the few systematic efforts in analyzing the relative contributions of terms of e-EoS. Tan et al. ${ }^{66}$ showed a very small analysis for the e-SAFT1 model (model which was later revised). A single point calculation is shown for $\mathrm{NaCl}$-water at 25 and 3.26 molality. In their model and for single point calculation, dispersion and association terms are quite large (and negative in residual Helmholtz energy), counterbalancing a smaller positive hard sphere term and an even smaller negative ion term. This is a model without a Born term. These results are rather different from the previous analyses and they could be very well attributed to over-parameterization of the model.

\section{Conclusions}

We have presented a modeling study of ACI in aqueous solutions by using the II+IW (ion-ion and ion-water) theory in various forms and the e-CPA EoS. Although the II+IW models are simplified and the performance is not perfect, they can represent the basic interactions present in electrolyte solutions. In many cases the results are in qualitative agreement with experimental data. It is shown that both II \& IW combinations are needed as they are typically of equal importance (magnitude). Moreover, the results shown are predictive as no ACI are used in parameter estimation 
in any of the models studied. The ion radius, the Born radius, the relative static permittivity and other parameters are critical in the calculations with the II+IW models and e-CPA. More investigations are needed for the study of ACI. It is believed that such studies as well as an analysis of the magnitude of the terms of the electrolyte models can result in an understanding of electrolyte theories and developments towards better approaches.

\section{Acknowledgments}

The authors acknowledge the support by the Department of Chemical and Biochemical Engineering, Technical University of Denmark and China Scholarship (No. 201608320224). The authors are grateful to the European Research Council (ERC) for funding of this research under the European Union's Horizon 2020 research and innovation program (grant agreement No. 832460), ERC Advanced Grant project "New Paradigm in Electrolyte Thermodynamics".

The authors also thank Professor Juan H. Vera for many useful suggestions.

\section{Supporting Information}

Supporting Information consists of three parts: Experimental Data Collection (Section A); Modelling Parameters (Section B); Basic Thermodynamic Equations (Section C). 


\section{Author Information}

Corresponding author:

*E-mail address: gk@kt.dtu.dk, Tel.: 0045-45252859. 


\section{References}

(1) Vera, J. H.; Wilczek-Vera, G. Reference Module in Chemistry, Molecular Sciences and Chemical Engineering: General Aspects of Ionic Activities in Aqueous Solutions. McGill University: Montreal, 2018.

(2) Kontogeorgis, G. M.; Folas, G. K. Thermodynamic models for industrial applications: from classical and advanced mixing rules to association theories. John Wiley and Sons: Great Britain, 2010; Vol. 15, pp 461-523.

(3) Tan, S. P.; Adidharma, H.; Radosz, M. Recent advances and applications of statistical associating fluid theory. Industrial \& Engineering Chemistry Research 2008, 47(21), 8063-8082.

(4) Thomsen, K. Electrolyte solutions: Thermodynamics, crystallization, separation methods. Technical University of Denmark: Copenhagen, 2009; Vol. 6, pp 35-53.

(5) Jiang, J.; Prausnitz, J. M. Equation of state for thermodynamic properties of chain fluids near-to and far-from the vapor-liquid critical region. The Journal of chemical physics 1999, 111(13), 5964-5974.

(6) Kielland, J. Individual activity coefficients of ions in aqueous solutions. Journal of the American Chemical Society 1937, 59(9), 1675-1678.

(7) Eisenman, G. Glass electrodes for hydrogen and other cations: principles and practice. Marcel Dekker: New York, 1967; Vol. 6, pp 174-218.

(8) Rodil, E.; Vera, J. Individual activity coefficients of chloride ions in aqueous solutions of $\mathrm{MgCl}$, $\mathrm{CaCl} 2$ and $\mathrm{BaCl} 2$ at $298.2 \mathrm{~K}$. Fluid phase equilibria 2001, 187, 15-27.

(9) Wilczek-Vera, G.; Rodil, E.; Vera, J. H. On the activity of ions and the junction potential: Revised values for all data. Aiche Journal 2004, 50(2), 445-462.

(10) Wilczek-Vera, G.; Rodil, E.; Vera, J. H. Towards accurate values of individual ion activities : Additional data for $\mathrm{NaCl}, \mathrm{NaBr}$ and $\mathrm{KCl}$, and new data for $\mathrm{NH} 4 \mathrm{Cl}$. Fluid Phase Equilibria 2006, 241(1), 59-69.

(11) Vera, J. H.; Wilczek-Vera, G. Classical Thermodynamics of Fluid Systems: Principles and Applications. CRC Press: New York, 2016; Vol. 27, pp 319-358.

(12) Arce, A.; Wilczek-Vera, G.; Vera, J. H. Activities of aqueous Na+ and Cl- ions from homoionic measurements with null junction potentials at different concentrations. Chemical Engineering Science 2007, 62(14), 3849-3857.

(13) Liu, J.-L.; Eisenberg, B. Poisson-Fermi modeling of ion activities in aqueous single and mixed electrolyte solutions at variable temperature. The Journal of chemical physics 2018, 148(5), 054501.

(14) Vincze, J.; Valiskó, M.; Boda, D. The nonmonotonic concentration dependence of the mean activity coefficient of electrolytes is a result of a balance between solvation and ion-ion correlations. The Journal of chemical physics 2010, 133(15), 154507.

(15) Vincze, J.; Valiskó, M.; Boda, D. Response to "Comment on 'The nonmonotonic concentration dependence of the mean activity coefficient of electrolytes is a result of a balance between solvation and ion-ion correlations'"'J. Chem. Phys. 134, 157101 (2011)]. The Journal of chemical physics 2011, 134(15), 157102.

(16) Valiskó, M.; Boda, D. Unraveling the behavior of the individual ionic activity coefficients on the basis of the balance of ion-ion and ion-water interactions. The Journal of Physical Chemistry B 2015, $119(4), 1546-1557$.

(17) Valiskó, M.; Boda, D. Activity coefficients of individual ions in $\mathrm{LaCl} 3$ from the II+ IW theory. 
Molecular Physics 2017, 115(9-12), 1245-1252.

(18) Fraenkel, D. Single-ion activity: experiment versus theory. The Journal of Physical Chemistry B 2012, 116(11), 3603-3612.

(19) Liu, J.-L.; Eisenberg, B. Poisson-Fermi model of single ion activities in aqueous solutions. Chemical Physics Letters 2015, 637, 1-6.

(20) Liu, J.-L.; Li, C.-L. A generalized Debye-Hückel theory of electrolyte solutions. AIP Advances 2019, $9(1), 015214$.

(21) Chen, D. P.; Barcilon, V.; Eisenberg, R. S. Constant fields and constant gradients in open ionic channels. Biophysical journal 1992, 61(5), 1372-1393.

(22) Eisenberg, R.; Kl/osek, M.; Schuss, Z. Diffusion as a chemical reaction: Stochastic trajectories between fixed concentrations. The Journal of chemical physics 1995, 102(4), 1767-1780.

(23) Liu, J.-L.; Eisenberg, B. Correlated ions in a calcium channel model: a Poisson-Fermi theory. The Journal of Physical Chemistry B 2013, 117(40), 12051-12058.

(24) Liu, J.-L.; Eisenberg, B. Poisson-Nernst-Planck-Fermi theory for modeling biological ion channels. The Journal of chemical physics 2014, 141(22), 12B640_1.

(25) Liu, J.-L.; Eisenberg, B. Numerical methods for a Poisson-Nernst-Planck-Fermi model of biological ion channels. Physical Review E 2015, 92(1), 012711.

(26) Valiskó, M.; Kristóf, T.; Gillespie, D.; Boda, D. A systematic Monte Carlo simulation study of the primitive model planar electrical double layer over an extended range of concentrations, electrode charges, cation diameters and valences. AIP Advances 2018, 8(2), 025320.

(27) Born, M. Volumen und hydratationswärme der ionen. Zeitschrift für Physik A Hadrons and Nuclei 1920, $1(1), 45-48$.

(28) Shilov, I. Y.; Lyashchenko, A. K. The Role of Concentration Dependent Static Permittivity of Electrolyte Solutions in the Debye-Hückel Theory. The Journal of Physical Chemistry B 2015, 119(31), 10087-10095.

(29) Valiskó, M.; Boda, D. Comment on "The Role of Concentration Dependent Static Permittivity of Electrolyte Solutions in the Debye-Hückel Theory". The Journal of Physical Chemistry B 2015, 119(44), 14332-14336.

(30) Lebowitz, J.; Percus, J. Mean spherical model for lattice gases with extended hard cores and continuum fluids. Physical Review 1966, 144(1), 251.

(31) Valiskó, M.; Boda, D. The effect of concentration-and temperature-dependent dielectric constant on the activity coefficient of $\mathrm{NaCl}$ electrolyte solutions. The Journal of chemical physics 2014, 140(23), 234508.

(32) Maribo-Mogensen, B.; Thomsen, K.; Kontogeorgis, G. M. An electrolyte CPA equation of state for mixed solvent electrolytes. AIChE Journal 2015, 61(9), 2933-2950.

(33) Debye, P.; Huckel, E. Zur Theorie der Elektrolyte. I. Gefrierpunktserniedrigung und verwandte Erscheinungen. Physikalische Zeitschrift 1923, 24, 185-206.

(34) Arfken, G. B.; Weber, H. J. Mathematical Methods for Physicists. Elsevier Academic Press: Burlington San Diego, 2005; Vol. 1, pp 79-82.

(35) Shavit, A.; Gutfinger, C. Thermodynamics: from concepts to applications. CRC Press: New York, 2008; Vol. 2, pp 5-20.

(36) Robinson, R. A.; Stokes, R. H. electrolyte solutions. Courier Corporation: North Chelmsford, 2002; Vol. 9, pp 223-252.

(37) Kontogeorgis, G. M.; Voutsas, E. C.; Yakoumis, I. V.; Tassios, D. P. An equation of state for 
associating fluids. Industrial \& engineering chemistry research 1996, 35(11), 4310-4318.

(38) Sun, L.; Liang, X.; Von Solms, N.; Kontogeorgis, G. M. Modeling Tetra-n-butyl ammonium halides aqueous solutions with the electrolyte cubic plus association equation of state. Fluid Phase Equilibria 2019, 486, 37-47.

(39) Sun, L.; Kontogeorgis, G. M.; von Solms, N.; Liang, X. Modeling of Gas Solubility Using the Electrolyte Cubic Plus Association Equation of State. Industrial \& Engineering Chemistry Research 2019, 58(37), 17555-17567.

(40) Hückel, E. Zur theorie konzentrierterer wässeriger Lösungen starker elektrolyte. Physikalische Zeitschrift 1925, 26, 93-147.

(41) Shilov, I. Y.; Lyashchenko, A. K. Modeling activity coefficients in alkali iodide aqueous solutions using the extended Debye-Hückel theory. Journal of Molecular Liquids 2017, 240, 172-178.

(42) Shilov, I. Y.; Lyashchenko, A. K. Comment on "The Debye-Hückel theory and its importance in modeling electrolyte solutions". Fluid Phase Equilibria 2019, 485, 248-250.

(43) Shilov, I. Y.; Lyashchenko, A. K. Anion-Specific Effects on Activity Coefficients in Aqueous Solutions of Sodium Salts: Modeling with the Extended Debye-Hückel Theory. Journal of Solution Chemistry 2019, 48(2), 234-247.

(44) Kontogeorgis, G. M.; Maribo-Mogensen, B.; Thomsen, K. The Debye-Hückel theory and its importance in modeling electrolyte solutions. Fluid Phase Equilibria 2018, 462, 130-152.

(45) Hubbard, J.; Onsager, L.; Van Beek, W.; Mandel, M. Kinetic polarization deficiency in electrolyte solutions. Proceedings of the National Academy of Sciences 1977, 74(2), 401-404.

(46) Fawcett, W. R.; Tikanen, A. C. Role of solvent permittivity in estimation of electrolyte activity coefficients on the basis of the mean spherical approximation. The Journal of Physical Chemistry 1996, 100(10), 4251-4255.

(47) Tikanen, A. C.; Fawcett, W. R. The role of solvent permittivity in estimation of electrolyte activity coefficients for systems with ion pairing on the basis of the mean spherical approximation. Berichte der Bunsengesellschaft für physikalische Chemie 1996, 100(5), 634-640.

(48) Malasics, A.; Gillespie, D.; Boda, D. Simulating prescribed particle densities in the grand canonical ensemble using iterative algorithms. The Journal of chemical physics 2008, 128(12), 124102.

(49) Young, J. M.; Panagiotopoulos, A. Z. System-size dependence of electrolyte activity coefficients in molecular simulations. The Journal of Physical Chemistry B 2018, 122(13), 3330-3338.

(50) Marcus, Y. Ionic radii in aqueous solutions. Chemical Reviews 1988, 88(8), 1475-1498.

(51) Shannon, R. D. Revised effective ionic radii and systematic studies of interatomic distances in halides and chalcogenides. Acta crystallographica section A: crystal physics, diffraction, theoretical and general crystallography 1976, 32(5), 751-767.

(52) Novotny, P.; Sohnel, O. Densities of binary aqueous solutions of 306 inorganic substances. Journal of Chemical and Engineering Data 1988, 33(1), 49-55.

(53) Hernández-Luis, F.; Amado-González, E.; Esteso, M. A. Activity coefficients of $\mathrm{NaCl}$ in trehalose-water and maltose-water mixtures at $298.15 \mathrm{~K}$. Carbohydrate research 2003, 338(13), 1415-1424.

(54) Schneider, A. C.; Pasel, C.; Luckas, M.; Schmidt, K.; Herbell, J. D. Bestimmung von Ionenaktivitätskoeffizienten in wässrigen Lösungen mit Hilfe ionenselektiver Elektroden. Chemie Ingenieur Technik 2003, 75(3), 244-249.

(55) Rard, J. A.; Habenschuss, A.; Spedding, F. H. A review of the osmotic coefficients of aqueous calcium chloride at 25. degree. C. Journal of Chemical and Engineering Data 1977, 22(2), 180-186. 
(56) Rard, J. A.; Miller, D. G. Isopiestic determination of the osmotic and activity coefficients of aqueous magnesium chloride solutions at 25. degree. C. Journal of Chemical and Engineering Data 1981, 26(1), 38-43.

(57) Hurlen, T. Single-ion activities of sodium sulfate in aqueous solution. Acta chemica scandinavica. Series A. Physical and inorganic chemistry 1983, 37(9), 739-742.

(58) Hurlen, T. Single-ion activities of lanthanum chloride in aqueous solution. Acta Chem. Scand. A 1983, 37(10).

(59) Onufriev, A. The generalized Born model: its foundation, applications, and limitations. Departments of Computer Science and Physics, Blacksburg, Virginia, USA 2010.

(60) Kistenmacher, H.; Prausnitz, J. M.; Vortmeyer, D. An equation of state for systems containing electrolytes and nonelectrolytes. Chemical Engineering and Processing: Process Intensification 1991, 29(3), 139-146.

(61) Inchekel, R.; de Hemptinne, J.-C.; Fürst, W. The simultaneous representation of dielectric constant, volume and activity coefficients using an electrolyte equation of state. Fluid Phase Equilibria 2008, 271(1-2), 19-27.

(62) Ahmed, S.; Ferrando, N.; de Hemptinne, J.-C.; Simonin, J.-P.; Bernard, O.; Baudouin, O. Modeling of mixed-solvent electrolyte systems. Fluid Phase Equilibria 2018, 459, 138-157.

(63) Shahriari, R.; Dehghani, M. R. Prediction of thermodynamic properties of aqueous electrolyte solutions using equation of state. AIChE Journal 2017, 63(11), 5083-5097.

(64) Selam, M. A.; Economou, I. G.; Castier, M. A thermodynamic model for strong aqueous electrolytes based on the eSAFT-VR Mie equation of state. Fluid Phase Equilibria 2018, 464, 47-63.

(65) Zúñiga-Hinojosa, M. A.; Martínez, J.; García-Sánchez, F.; Macías-Salinas, R. Modeling the Hydrate Dissociation Pressure of Light Hydrocarbons in the Presence of Single $\mathrm{NaCl}, \mathrm{KCl}$, and $\mathrm{CaCl} 2$ Aqueous Solutions Using a Modified Equation of State for Aqueous Electrolyte Solutions with Partial Ionization. Industrial \& Engineering Chemistry Research 2019, 58(27), 12369-12391.

(66) Tan, S. P.; Adidharma, H.; Radosz, M. Statistical associating fluid theory coupled with restricted primitive model to represent aqueous strong electrolytes. Industrial \& engineering chemistry research 2005, 44(12), 4442-4452. 
For Table of Contents Only

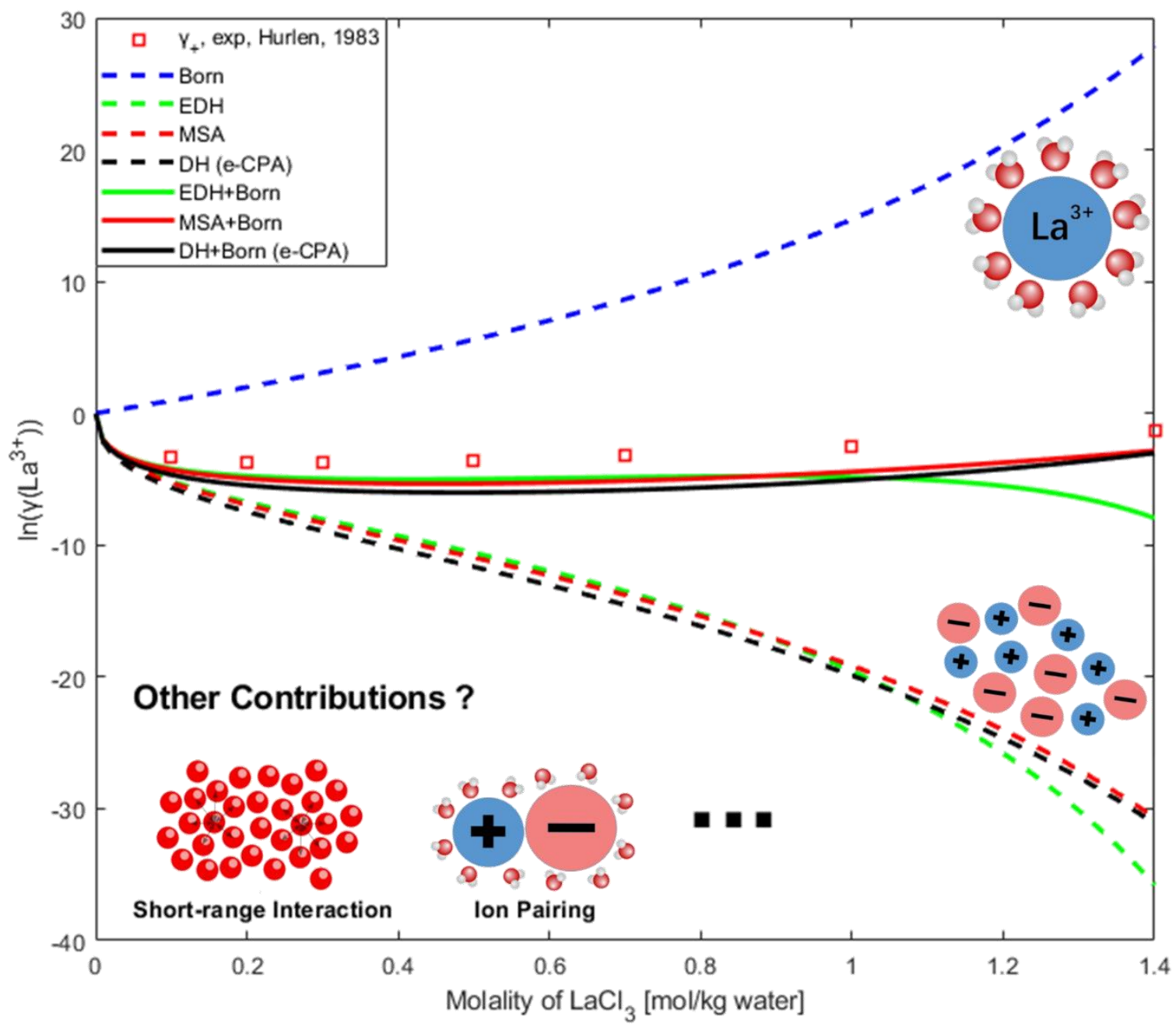

